\title{
MODELOS PARA PROPORÇÕES COM SUPERDISPERSÃO E EXCESSO DE ZEROS - UM PROCEDIMENTO BAYESIANO
}

\author{
ADRIANO FERRETI BORGATTO
}

\begin{abstract}
Tese apresentada à Escola Superior de Agricultura "Luiz de Queiroz", Universidade de São Paulo, para obtenção do título de Doutor em Agronomia, Área de Concentração: Estatística e Experimentação Agronômica.
\end{abstract}

P I R A C I C A B A

Estado de São Paulo - Brasil

Maio - 2004 


\title{
MODELOS PARA PROPORÇÕES COM SUPERDISPERSÃO E EXCESSO DE ZEROS - UM PROCEDIMENTO BAYESIANO
}

\author{
ADRIANO FERRETI BORGATTO \\ Estatístico \\ Orientadora: $\operatorname{Prof}^{\underline{a}} \operatorname{Dr}^{\underline{a}}$ CLARICE GARCIA BORGES DEMÉTRIO
}

Tese apresentada à Escola Superior de Agricultura "Luiz de Queiroz", Universidade de São Paulo, para obtenção do título de Doutor em Agronomia, Área de Concentração: Estatística e Experimentação Agronômica.

P I R A C I C A B A

Estado de São Paulo - Brasil

Maio - 2004 


\section{Dados Internacionais de Catalo gação na Publicação (CIP) DNISÃOO DE BIBLIOTECA E DOCUMIENTAÇÄO - ESALQ/USP}

Borgatto, Adriano Ferreti

Modelos para proporçỗes com superdispersẫo e excesso de zeros - um procedimento baye siano / Adriano Ferreti Borgatto. - - Piracicaba, 2004.

$90 \mathrm{p}$.

Tese (doutorado) - - Escola Superior de Agricultura Luiz de Queiroz, 2004

Bibliografia.

1. Controle biológico 2. Inferência bayesiana (Inferência estatística) 3. Modelos line are s generalizados I.Título

CDD 519.542

"Permitida a cópia total ou parcial deste documento, desde que citada a fonte - 0 autor" 


\section{DEDICATÓRIA}

A

DEUS

força maior de todo ser humano.

Aos meus pais,

Vicente Waldyr Borgatto e

Ana Cleide Ferreti Borgatto,

os inúmeros bons exemplos que propiciaram, os quais me impulsionaram no término de mais uma fase em minha vida.

À minha noiva Fabiana o amor, o apoio e, principalmente a compreensão nos momentos em que estive ausente. 


\section{AGRADECIMENTOS}

À minha orientadora $\operatorname{Prof}^{\underline{a}} \operatorname{Dr}^{\underline{a}}$ Clarice Garcia Borges Demétrio o ensinamento, a contribuição, a dedicação, a amizade e a confiança depositada.

À Prof $\underline{\underline{a}}$ Dr $\underline{\underline{a}}$ Roseli Aparecida Leandro a amizade, dedicação e sua valiosa contribuição na realização deste trabalho.

Aos professores Dr. Silvio S. Zocchi, Décio Barbin e Sônia M. de S. Piedade o carinho e a convivência.

Ao CNPQ o fundamental suporte financeiro concedido.

Aos colegas e amigos do doutorado e mestrado, em especial a Silvano Cesar da Costa (UEL - Londrina-PR), João Mauricio de Araújo Mota (UFC - Fortaleza-CE), Suely Ruiz Giolo (UFPR - Curitiba-PR), Antonio Carlos Fonseca Pontes (UFAC - Rio Branco-AC), Renato Ribeiro de Lima (UFV - Viçosa-MG), Antonio Willians Moita (EMBRAPA - Brasília-DF), Juliana Garcia Cespedes, Denise Nunes Viola, Genevile Carife Bergamo, Elizabeth Strapasson (UEL - Londrina-PR) e Ana Maria Souza de Araújo (UFC - Fortaleza-CE) a força, a amizade, a troca de conhecimentos e atenção recebida em todos os momentos.

Às minhas amigas dona Diva, Rosa e Iza, a atenção, o carinho e a amizade.

Aos professores, funcionários do Departamento de Ciências Exatas da ESALQ/USP e a todos que me propiciaram condições para a realização deste trabalho. 


\section{SUMÁRIO}

Página

LISTA DE FIGURAS . . . . . . . . . . . . . . . . . . . . . vii

LISTA DE TABELAS ...................... . . . ix

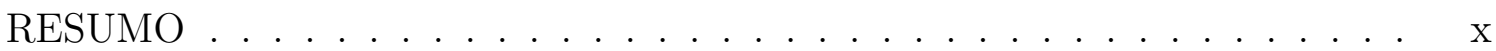

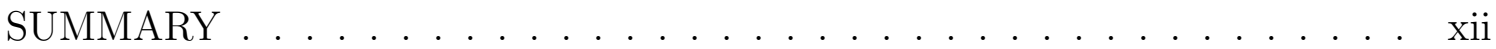

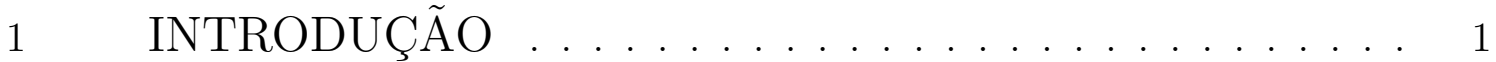

2 REVISÃO DE LITERATURA ............... 3

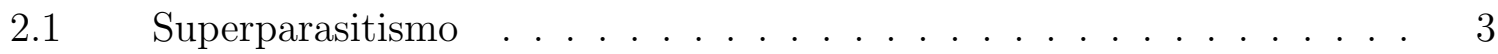

2.2 Modelos Lineares Generalizados . . . . . . . . . . . . . . . 4

2.3 Modelos para dados com superdispersão . . . . . . . . . . . . . . . 10

2.4 Modelos para dados com excesso de zeros . . . . . . . . . . . . . 14

2.4.1 Métodos de Estimação . . . . . . . . . . . . . . . . 20

2.4.1.1 Clássicos . . . . . . . . . . . . . . . . . . . . . . 20

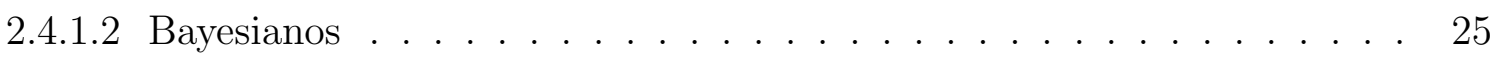

2.5 Diagnósticos para modelos lineares generalizados . . . . . . . . . . . 29

3 MATERIAL E MÉTODOS . . . . . . . . . . . . 32

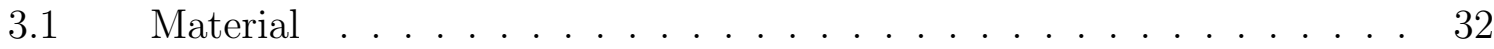

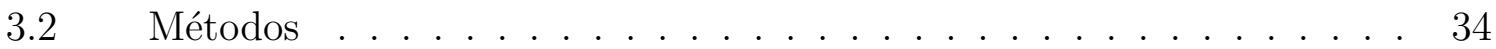

3.2.1 Modelo binomial com superdispersão . . . . . . . . . . . . . . . . . 34

3.2.2 Modelo binomial truncado superdisperso . . . . . . . . . . . . . . 40

3.2.3 Modelo binomial inflacionado de zeros superdisperso . . . . . . . . . 43

4 RESULTADOS E DISCUSSÃO . . . . . . . . . . . 51

4.1 Modelo binomial superdisperso . . . . . . . . . . . . . . 51 
4.2 Modelo binomial truncado superdisperso . . . . . . . . . . . . 57

4.3 Modelo binomial inflacionado de zeros superdisperso . . . . . . . . . . 62

4.4 Comparação entre os modelos binomial, binomial truncado e ZIB . . . 68

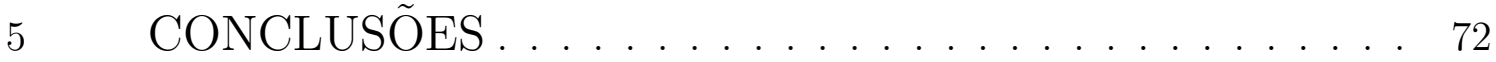

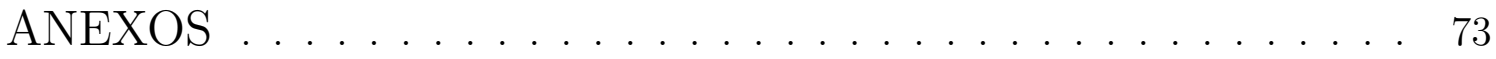

REFERÊNCIAS BIBLIOGRÁFICAS . . . . . . . . . . . . . 84 


\section{LISTA DE FIGURAS}

Página

1 Proporção de ovos de A. kuehniella parasitados, em função do número de fêmeas do T. galloi (o diâmetro do ponto reflete a grandeza da frequência) 33

2 Trajetória da cadeia ao longo das iterações e a aproximação da densidade a posteriori de $\phi$ para o modelo binomial . . . . . . . . . . . . . . . . . . 51

3 Valores observados, preditos e intervalos de credibilidade a $95 \%$ de probabilidade para o modelo binomial superdisperso considerando o preditor linear como um polinômio de quarto grau . . . . . . . . . . . . . 54

4 Trajetória da cadeia ao longo das iterações e a aproximação da densidade a posteriori de $\beta_{0}$ para o modelo binomial . . . . . . . . . . . . 55

5 Trajetória da cadeia ao longo das iterações e a aproximação da densidade a posteriori de $\beta_{1}$ para o modelo binomial . . . . . . . . . . . 55

6 Trajetória da cadeia ao longo das iterações e a aproximação da densidade a posteriori de $\beta_{2}$ para o modelo binomial . . . . . . . . . . . 56

7 Trajetória da cadeia ao longo das iterações e a aproximação da densidade a posteriori de $\beta_{3}$ para o modelo binomial . . . . . . . . . . . 56

8 Trajetória da cadeia ao longo das iterações e a aproximação da densidade a posteriori de $\beta_{4}$ para o modelo binomial $\ldots \ldots \ldots$. . . . . . 56

9 Trajetória da cadeia ao longo das iterações e a aproximação da densidade a posteriori de $\phi$ para o modelo binomial truncado . . . . . . . . . . 57

10 Valores observados, preditos e intervalos de credibilidade a $95 \%$ de probabilidade para o modelo binomial truncado superdisperso considerando o preditor linear como um polinômio de terceiro grau . . . . . . . . . . . 60 
11 Trajetória da cadeia ao longo das iterações e a aproximação da densidade a posteriori de $\beta_{0}$ para o modelo binomial truncado . . . . . . . . . . . 60

12 Trajetória da cadeia ao longo das iterações e a aproximação da densidade a posteriori de $\beta_{1}$ para o modelo binomial truncado . . . . . . . . . . . . 61

13 Trajetória da cadeia ao longo das iterações e a aproximação da densidade a posteriori de $\beta_{2}$ para o modelo binomial truncado . . . . . . . . . . . . 61

14 Trajetória da cadeia ao longo das iterações e a aproximação da densidade a posteriori de $\beta_{3}$ para o modelo binomial truncado . . . . . . . . . . . . 61

15 Trajetória da cadeia ao longo das iterações e a aproximação da densidade a posteriori de $\phi$ para o modelo ZIB . . . . . . . . . . . . . . . . . . 62

16 Valores observados, preditos e intervalos de credibilidade a $95 \%$ de probabilidade para o modelo ZIB superdisperso considerando os preditores lineares como polinômios de terceiro grau para $\boldsymbol{p}$ e constante para $\boldsymbol{w}$. . 65

17 Trajetória da cadeia ao longo das iterações e a aproximação da densidade a posteriori de $\beta_{0}$ para o modelo ZIB . . . . . . . . . . . . . . . 66

18 Trajetória da cadeia ao longo das iterações e a aproximação da densidade a posteriori de $\beta_{1}$ para o modelo ZIB . . . . . . . . . . . . . . . 66

19 Trajetória da cadeia ao longo das iterações e a aproximação da densidade a posteriori de $\beta_{2}$ para o modelo ZIB . . . . . . . . . . . . . . . . . 67

20 Trajetória da cadeia ao longo das iterações e a aproximação da densidade a posteriori de $\beta_{3}$ para o modelo ZIB . . . . . . . . . . . . . . . . . 67

21 Trajetória da cadeia ao longo das iterações e a aproximação da densidade a posteriori de $\gamma_{0}$ para o modelo ZIB . . . . . . . . . . . . . . . . 67

22 Comparação entre os valores preditos pelas distribuições e o valor observado 68

23 Porcentagem observada e estimada de ovos não parasitados, segundo as distribuições binomial e ZIB . . . . . . . . . . . . . . . . . . . 69 


\section{LISTA DE TABELAS}

Página

1 Interpretação do fator de Bayes . . . . . . . . . . . . . . . . . . . 40

2 Valores do DIC e respectivos números de parâmetros efetivos estimados para o modelo binomial . . . . . . . . . . . . . . . . 52

3 Valores da verossimilhança marginal para o modelo binomial . . . . . . 53

4 Sumários a posteriori do modelo binomial superdisperso, considerando como preditor linear como um polinômio de quarto grau . . . . . . . . 53

5 Valores do DIC e respectivos números efetivos de parâmetros estimados para o modelo binomial truncado . . . . . . . . . . . . . . 58

6 Valores da verossimilhança marginal para o modelo binomial truncado . 58

7 Sumários a posteriori do modelo binomial truncado superdisperso, considerando o preditor linear como um polinômio de terceiro grau . . . . 59

8 Valores do DIC e respectivos números efetivos de parâmetros estimados para o modelo binomial inflacionado de zeros . . . . . . . . . . . . 63

9 Valores da verossimilhança marginal para o modelo ZIB . . . . . . . . . . 64

10 Sumários a posteriori do modelo binomial inflacionado de zeros superdisperso, considerando os preditors linear como um polinômio de terceiro

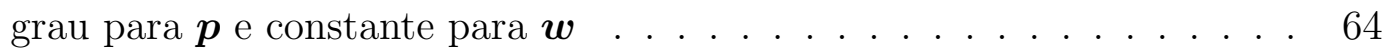

11 Número médio esperado de ovos parasitados usando diferentes números de fêmeas ........................... 68

12 Valores das verossimilhanças marginais para os modelos binomial, binomial truncado e ZIB . . . . . . . . . . . . . . . . . . . 70 


\title{
MODELOS PARA PROPORÇÕES COM SUPERDISPERSÃO E EXCESSO DE ZEROS - UM PROCEDIMENTO BAYESIANO
}

\author{
Autor: ADRIANO FERRETI BORGATTO \\ Orientadora: $\operatorname{Prof}^{\underline{a}} \operatorname{Dr}^{\underline{a}}$ CLARICE GARCIA BORGES DEMÉTRIO
}

\section{RESUMO}

Neste trabalho, três modelos foram ajustados a um conjunto de dados obtido de um ensaio de controle biológico para Diatraea saccharalis, uma praga comum em plantações de cana-de-açúcar. Usando a distribuição binomial como modelo de probabilidade, um ajuste adequado não pode ser obtido, devido à superdispersão gerada pela variabililidade dos dados e pelo excesso de zeros. Nesse caso, o modelo binomial inflacionado de zeros (ZIB) superdisperso é mais flexível e eficiente para a modelagem desse tipo de dados. Entretanto, quando o interesse maior está sobre os valores positivos das proporções, pode-se utilizar o modelo binomial truncado superdisperso. Uma abordagem alternativa eficiente que foi utilizada para a modelagem desse tipo de dados foi a Bayesiana, sendo o ajuste do modelo realizado usando as técnicas de simulação Monte Carlo em Cadeias de Markov, através do algoritmo Metropolis-Hastings e a seleção dos modelos foi feita usando o DIC (Deviance 
Information Criterion) e o fator de Bayes. Os modelos foram implementados no procedimento IML (Iteractive Matrix Linear) do programa SAS (Statistical Analysis System) e no programa WinBUGS e a convergência das estimativas foi verificada através da análise gráfica dos valores gerados e usando os diagnósticos de Raftery \& Lewis e de Heidelberger \& Welch, implementado no módulo CODA do programa R. 


\title{
MODELS FOR ZERO-INFLATED AND OVERDISPERSED PROPORTION DATA - A BAYESIAN APPROACH
}

\author{
Author: ADRIANO FERRETI BORGATTO \\ Adviser: Prof $\underline{a}^{\underline{a}} \operatorname{Dr}^{\underline{a}}$ CLARICE GARCIA BORGES DEMÉTRIO
}

SUMMARY

In general the standard binomial regression models do not fit well to proportion data from biological control assays, manly when there is excess of zeros and overdispersion. In this work a zero-inflated binomial model is applied to a data set obtained from a biological control assay for Diatraea saccharalis, a commom pest in sugar cane. A parasite (Trichogramma galloi) was put to parasitize 128 eggs of the Anagasta kuehniella, an economically suitable alternative host (Parra, 1997), with a variable number of female parasites $(2,4,8, \ldots, 128)$, each with 10 replicates in a completely randomized experiment. When interest is only in the positive proportion data, a model can be based on the truncated binomial distribution. A Bayesian procedure was formulated using a simulation technique (Metropolis Hastings) for estimation of the posterior parameters of interest. The convergence of the Markov 
Chain generated was monitored by visualization of the trace plot and using Raftery \& Lewis and Heidelberg \& Welch diagnostics presented in the module CODA of the software R. 


\section{INTRODUÇÃO}

O controle biológico aplicado (CBA) é um importante ramo da Entomologia, que busca métodos para controle de pragas sem prejudicar o meio ambiente e o ecossistema, utilizando inimigos naturais da praga (parasitóides e/ou predadores). Essa área teve um grande avanço após a década de 60, com o desenvolvimento de técnicas de criação de insetos de forma intensiva. Especificamente no Brasil, uma praga que vem sendo combatida através de CBA é a broca-da-cana (Diatraea saccharalis), que é a principal praga da cana-de-açúcar de nosso país. Em 1983, no Estado de São Paulo, gerou prejuízos estimados em $U \$ 119$ milhões, sendo que hoje esse prejuízo reduziu-se para $U \$ 20$ milhões (Folha de São Paulo, 14/08/2003).

A broca-da-cana, ainda hoje, é considerada letal aos canaviais porque os seus prejuízos podem ser diretos ou indiretos e esses últimos são considerados os mais preocupantes. Diretamente, a broca pode causar perda de peso do produto, pois alimenta-se do colmo, e quando isso ocorre no início do desenvolvimento da cultura, chega a causar a morte da planta. Os danos indiretos são causados porque a entrada da broca no colmo deixa aberturas para microrganismos que irão inverter a sacarose e contaminar o caldo, causando perdas, tanto de açúcar como de álcool na indústria. Em uma propriedade com produção média de 80 toneladas de cana por hectare, essas perdas podem chegar a 30 quilos de açúcar e 25 litros de álcool por hectare.

Botelho (1985) constatou que uma forma de combater a broca-da-cana

é através do uso de parasitóides de ovos ( Trichogramma galloi), tendo a vantagem de parasitar a broca-da-cana na sua fase inicial de desenvolvimento, evitando assim os danos causados pela lagarta, além de reduzir a infestação da praga na própria safra 
em que é liberada.

A utilização de parasitóides do gênero Trichogramma para o controle de D. saccharalis tem sido uma fonte de pesquisas do Laboratório de Biologia do Departamento de Entomologia da ESALQ/USP. O objetivo de muitas dessas pesquisas é a otimização de técnicas para a obtenção de parasitóides para liberações inundativas no campo (Parra, 1997). Devido ao alto custo de produção de ovos do D. saccharalis em laboratórios, os entomologistas optam por usar um hospedeiro alternativo (Anagasta kueniella) para parasitar os ovos.

Em alguns ensaios de controle biológico são observados dados na forma de proporções e os modelos comumente utilizados na análise envolvem a distribuição binomial. Ocorre, porém, que na maior parte dos casos, os dados apresentam uma variabilidade maior do que a prevista pela distribuição binomial tornando-se esses modelos inadequados para o ajuste. Um dos fatores que pode contribuir para isso é a presença de um excesso de zeros e nesse caso, modelos que levem isso em consideração devem ser utilizados (Ridout et al., 1998, Vieira, 1998 e Vieira et al., 2000).

Muitos aspectos desses modelos, porém, não estão completamente estudados, necessitando de estudos adicionais. Portanto, o presente trabalho tem como objetivos:

(i) fazer uma revisão de literatura sobre a modelagem de dados em que existe superdispersão e excesso de zeros;

(ii) usar procedimentos para estimação Bayesiana;

(iii) implementar os métodos em programas computacionais;

(iv) verificar a qualidade de ajuste dos modelos usando métodos Bayesianos;

(v) modelar os dados de um ensaio de controle biológico. 


\section{REVISÃO DE LITERATURA}

\subsection{Superparasitismo}

O superparasitismo, segundo Smith (1916) significa que vários parasitóides de uma mesma espécie atacam um único hospedeiro. Este fenômeno ocorre devido ao fato de que vários indivíduos de uma mesma espécie de parasitóide podem se desenvolver em um hospedeiro. Em alguns casos, entretanto, o hospedeiro morre prematuramente, antes que os excedentes sejam eliminados, e com isso, todos morrem.

O comportamento de evitar o superparasitismo foi observado em muitas espécies de parasitóides (Rogers, 1975), os quais se reproduzem colocando ovos em hospedeiros, tendo no máximo 1 ovo por hospedeiro desenvolvendo-se até a fase adulta. A vantagem desse tipo de parasitóide é a redução no desperdício de ovos.

A modelagem desse processo leva em consideração 5 etapas:

(i) a procura pelo hospedeiro e a ovoposição são considerados independentes;

(ii) o processo de procura conduz cada hospedeiro a um número aleatório de contatos com os parasitóides;

(iii) no primeiro contato entre hospedeiro e os parasitóides, sempre é colocado um único ovo;

(iv) a cada contato subseqüente ao primeiro, o parasitóide colocará, ou não, um único ovo e 
(v) o comportamento de evitar o superparasitismo ocorre a uma proporção fixada de encontros múltiplos, com probabilidade independente do número e da maturidade de parasitóides já presentes e de outros fatores ambientais ou fisiológicos.

Um modelo envolvendo esse processo, proposto por Bakker et al. (1967), considera o número de contatos entre fêmeas dos parasitóides ovopositoras e os hospedeiros tendo distribuição Poisson. Assume-se, ainda, que um ovo é colocado no primeiro contato com probabilidade 1 , mas que um mecanismo reduz a probabilidade de um ovo ser colocado em contatos subseqüentes. Morgan (1975) usou esse modelo para descrever a fertilização de ovos de ouriço do mar, sendo que o interesse era determinar o número de espermatozóides depositados em um único ovo. Uma variação desse modelo foi proposta por Rogers (1975) supondo que o número de contatos entre parasitóides e hospedeiros segue uma distribuição binomial condicional, mas o mecanismo não é alterado.

Modelos mais complexos em que a probabilidade de fuga do parasitóide é uma função do número de ovos presentes em um hospedeiro foram propostos por Bakker et al. (1972).

A seguir será feita uma consideração sobre modelos mais gerais que podem incluir variabilidade causada pelos mecanismos de parasitismo, através de inclusão de parâmetros para modelar a superdispersão.

\subsection{Modelos Lineares Generalizados}

Metodologias para modelagem de dados na forma de proporções e de contagem foram propostas antes da década de 70 (Finney, 1947; Grizzle et al., 1969), sendo que a unificação do procedimento de modelagem foi proposta por Nelder \& Wedderburn (1972), sob o nome de Modelos Lineares Generalizados (MLG).

Nelder \& Weddeburn (1972) definiram MLG, para uma amostra de $n$ observações de uma variável aleatória $Y$, a partir de três componentes (Demétrio, 
2001):

(i) Parte aleatória: a variável aleatória independente $Y_{i}, i=1, \ldots, n$, tem distribuição pertencente à família exponencial na forma canônica com média $\mu_{i} \mathrm{e}$ parâmetro de dispersão constante $\phi>0$;

(ii) Parte sistemática: associam-se ao modelo variáveis explanatórias $\boldsymbol{x}_{i}^{T}=\left(x_{1 i}, x_{2 i}, \ldots, x_{q i}\right), i=1, \ldots, n$, com o vetor de preditores lineares dado por

$$
\eta_{i}=\boldsymbol{x}_{i}^{T} \boldsymbol{\beta}
$$

sendo $\boldsymbol{\beta}$ um vetor de $q$ parâmetros desconhecidos e $\boldsymbol{x}_{i}^{T}$ a matriz de delineamento $n \times q$, ou a matriz de covariáveis, em que $x_{1 i}=1$ para todo $i$;

(iii) Função de Ligação: uma função que associa a parte aleatória, dada em (i), a parte sistemática, dado em (ii) é,

$$
g\left(\mu_{i}\right)=\eta_{i}=\boldsymbol{x}_{i}^{T} \boldsymbol{\beta}
$$

A escolha da função de ligação depende da distribuição definida em (i). Por exemplo, a função de ligação logística pode ser usada para a distribuição binomial.

A variável aleatória $Y_{i}$ tem distribuição pertencente à família exponencial na forma canônica, se a função de probabilidade puder ser escrita por

$$
f\left(y_{i} ; \theta_{i}, \phi\right)=\exp \left\{\frac{y_{i} \theta_{i}-b\left(\theta_{i}\right)}{a_{i}(\phi)}+c\left(y_{i}, \phi\right)\right\}
$$

em que $a(),. b($.$) e c($.$) são funções específicas, \theta_{i}$ é um parâmetro natural ou canônico. Em geral, usa-se $a_{i}(\phi)=\frac{\phi}{w_{i}}$ sendo que $\phi$ é chamado de parâmetro de escala ou dispersão e $w_{i}$ é o peso a priori. O parâmetro canônico, $\theta_{i}$, é uma função da média e, portanto, pode ser relacionado ao preditor linear. Uma escolha natural da função de ligação é

$$
\theta_{i}=\eta_{i}=g\left(\mu_{i}\right)
$$


sendo conhecida como função de ligação canônica.

A média e a variância de $Y_{i}$ são dadas, respectivamente, por

$$
E\left(Y_{i}\right)=b^{\prime}\left(\theta_{i}\right)=\mu_{i}
$$

e

$$
\operatorname{Var}\left(Y_{i}\right)=a(\phi) b^{\prime \prime}\left(\theta_{i}\right)=a(\phi) V\left(\mu_{i}\right)
$$

sendo $b^{\prime}\left(\theta_{i}\right)$ e $b^{\prime \prime}\left(\theta_{i}\right)$ as derivadas de $1^{a}$ e $2^{a}$ ordens de $b\left(\theta_{i}\right)$ em relação a $\theta_{i}$, respectivamente. A função $b^{\prime \prime}\left(\theta_{i}\right)$ pode ser expressa por $V\left(\mu_{i}\right)$, pois essa depende apenas de $\mu_{i}$, sendo chamada de função de variância.

A estimação dos parâmetros pode ser feita pelo método de máxima verossimilhança. Considerando uma variável aleatória $Y_{i}$ com observações $\boldsymbol{y}^{\boldsymbol{T}}=\left(y_{1}, y_{2}, \ldots, y_{n}\right)$ de uma distribuição pertencente à família exponencial (1), a função de verossimilhança é dada por

$$
\begin{aligned}
L(\boldsymbol{\theta}, \phi ; \boldsymbol{y}) & =\prod_{i=1}^{n} f\left(y_{i} ; \theta_{i}, \phi\right) \\
& =\exp \left[\sum_{i=1}^{n}\left\{\frac{y_{i} \theta_{i}-b\left(\theta_{i}\right)}{a(\phi)}+c\left(y_{i}, \phi\right)\right\}\right]
\end{aligned}
$$

cujo logaritmo é dado por

$$
l(\boldsymbol{\theta}, \phi ; \boldsymbol{y})=\sum_{i=1}^{n}\left\{\frac{y_{i} \theta_{i}-b\left(\theta_{i}\right)}{a(\phi)}+c\left(y_{i}, \phi\right)\right\} .
$$

Através da análise de deviance, a análise de variância foi generalizada, incorporando os modelos log-lineares para tabelas de contingência, análise probit, regressão logística e regressão Poisson, ligando a análise de dados discretos e contínuos. Existe uma vasta literatura de modelos lineares generalizados, e, dentre outros, podem ser citados Cordeiro (1986), McCullagh \& Nelder (1989), Collett (1991), Demétrio (2001), Dobson (2001) e Paula (2002).

O ajuste de um modelo, com valores estimados $\hat{\boldsymbol{\mu}}$, a um conjunto de dados observados, $\boldsymbol{y}$, pode ser verificado através da estatística scaled deviance (Nelder \& Wedderburn, 1972), dada por

$$
S_{q}(\hat{\boldsymbol{\mu}}, \phi ; \boldsymbol{y})=-2\{l(\hat{\boldsymbol{\mu}}, \phi ; \boldsymbol{y})-l(\tilde{\boldsymbol{y}}, \phi ; \boldsymbol{y})\},
$$


sendo $q$ o número de parâmetros do modelo, $l(\hat{\boldsymbol{\mu}}, \phi ; \boldsymbol{y})$ o logaritmo da função de verossimilhança para o modelo sob ajuste e $l(\tilde{\boldsymbol{y}}, \phi ; \boldsymbol{y})$ o logaritmo da função de verossimilhança para o modelo saturado. Para o caso particular em que $\phi=1$, esta estatística reduz-se à deviance, isto é,

$$
D(\hat{\boldsymbol{\mu}} ; \boldsymbol{y})=-2\{l(\hat{\boldsymbol{\mu}} ; \boldsymbol{y})-l(\tilde{\boldsymbol{y}} ; \boldsymbol{y})\}
$$

Uma outra medida de ajuste alternativa é a estatística $X^{2}$ generalizada de Pearson, que tem a forma

$$
X^{2}=\sum_{i=1}^{n} \frac{\left(y_{i}-\hat{\mu}_{i}\right)^{2}}{\hat{V}\left(\hat{\mu}_{i}\right)}
$$

sendo $\hat{V}\left(\hat{\mu}_{i}\right)$ a função de variância estimada para a distribuição em estudo.

Para dados provenientes das distribuições binomial e Poisson, essa estatística é dada por

$$
X^{2}=\sum_{i=1}^{n} \frac{\left(O_{i}-E_{i}\right)^{2}}{E_{i}}
$$

sendo $O_{i}$ a frequência observada e $E_{i}$ a frequência esperada.

Pode-se mostrar que $S_{q}(\hat{\boldsymbol{\mu}}, \phi ; \boldsymbol{y})$ e $\frac{X^{2}}{\phi}$ têm distribuição $\chi_{n-q}^{2}$ exata, quando a distribuição é normal e distribuição $\chi_{n-q}^{2}$ assintótica, para as distribuições binomial e Poisson, sob determinadas condições de regularidade. Deseja-se selecionar um modelo que seja parcimonioso e explique bem os dados.

Portanto, para um modelo bem ajustado, espera-se que a deviance residual esteja próxima do número de graus de liberdade do resíduo do modelo. Uma maneira de obter uma deviance próxima do número de graus de liberdade é aumentar o número de parâmetros no modelo, mas com isso, aumenta-se também a complexidade na interpretação. O ideal é encontrar modelos simples com deviance moderada, situados entre os modelos mais complicados e os que se ajustam mal aos dados.

Outros critérios comumente usados para a seleção de modelos são o critério de informação de Akaike (AIC) e o critério de informação de Bayes (BIC), 
respectivamente propostos por Akaike (1973) e Schawarz (1978) e dados por

$$
A I C=-2 l(\hat{\boldsymbol{\mu}}, \phi ; \boldsymbol{y})+2 q
$$

e

$$
B I C=-2 l(\hat{\boldsymbol{\mu}}, \phi ; \boldsymbol{y})+q \log (n) .
$$

Nota-se que o AIC leva em consideração o número $q$ de parâmetros do modelo e o BIC o número $q$ de parâmetros e o número $n$ de observações. Os dois critérios são usados tanto no caso de modelos aninhados como não aninhados.

O melhor modelo é aquele que apresenta menor valor de um dos critérios utilizados (AIC ou BIC). Entretanto, segundo Lindsey (1997), o AIC é mais apropriado para dados provenientes de pequenas amostras.

Recentemente, Spiegelhalter et al. (1998) propuseram uma generalização do critério de informação de Akaike, para o ajuste dos modelos Bayesianos, usando métodos MCMC baseado na distribuição a posteriori da estatística da deviance, dada em $(2)$, em que $l(\tilde{\boldsymbol{y}} ; \boldsymbol{y})$ é alguma função de padronização dos dados, não tendo influência sobre a seleção dos modelos. De acordo com essas condições, o ajuste do modelo é resumido pela esperança a posteriori da deviance

$$
\bar{D}(\boldsymbol{\mu} ; \boldsymbol{y})=E_{\mu \mid y}[D(\boldsymbol{\mu} ; \boldsymbol{y})]
$$

enquanto que o número de parâmetros de um modelo é estimado pelo número efetivo de parâmetros $p_{D}$, dado por

$$
p_{D}=E_{\mu \mid y}[D(\boldsymbol{\mu} ; \boldsymbol{y})]-D\left(E_{\mu \mid y}[\boldsymbol{\mu}]\right)=\bar{D}(\boldsymbol{\mu} ; \boldsymbol{y})-D(\overline{\boldsymbol{\mu}} ; \boldsymbol{y})
$$

sendo $D(\overline{\boldsymbol{\mu}} ; \boldsymbol{y})$ a deviance, obtida fixando-se as estimativas dos parâmetros obtidas pela esperança a posteriori.

Com isso, os modelos podem ser comparados usando o critério de informação da deviance (DIC) definido como

$$
D I C=\bar{D}(\boldsymbol{\mu} ; \boldsymbol{y})+p_{D}=2 \bar{D}(\boldsymbol{\mu} ; \boldsymbol{y})-D(\overline{\boldsymbol{\mu}} ; \boldsymbol{y})
$$


sendo que o menor valor do DIC, indica um melhor ajuste do modelo.

Outro critério Bayesiano para comparação de modelos é o fator de Bayes (Kass \& Raftery, 1995) que é a razão das verossimilhanças marginais de dois modelos. A verossimilhança marginal é de grande importância na análise Bayesiana, quando o interesse está na discriminação dos modelos em estudo. Considerando um determinado modelo $M$, a verossimilhança marginal é definida por

$$
\pi(\boldsymbol{y} \mid M)=\int L(\boldsymbol{\mu} ; M) \pi(\boldsymbol{\mu} \mid M) d \boldsymbol{\mu}
$$

em que $L(\boldsymbol{\mu} ; M)$ é a função de verossimilhança para o modelo $M, \pi(\boldsymbol{\mu} \mid M)$ a distribuição a priori conjunta para os parâmetros do modelo $M$ e $\boldsymbol{\mu}$ o vetor de parâmetros do modelo.

Assim, dados dois modelos $M_{1}$ e $M_{2}$, o fator de Bayes, definido como a razão das verossimilhanças marginais desses modelos, é dado por

$$
B=\frac{\pi\left(\boldsymbol{y} \mid M_{1}\right)}{\pi\left(\boldsymbol{y} \mid M_{2}\right)}
$$

Dessa forma, o fator de Bayes é um valor numérico baseado apenas nos dados. Quando essa razão é maior do que um, ou seja $B>1$, o modelo $M_{1}$ ajusta-se melhor aos dados, caso contrário o modelo $M_{2}$ é melhor.

Para o caso em que se deseja comparar $n$ modelos, $M_{1}, M_{2}, \cdots, M_{n}$, escolhe-se o modelo que apresentar o maior valor da verossimilhança marginal.

Em algumas situações elementares envolvendo modelos simples, a verossimilhança marginal dada em (3) pode ser calculada analiticamente. Contudo, em geral, essa função é de difícil resolução devido ao cálculo das integrais e portanto, é necessário utilizar métodos alternativos para aproximá-las. Um dos métodos que pode ser utilizado é o método de Monte Carlo em Cadeia de Markov (MCMC). Utilizando esse método, gera-se uma amostra $\left(\boldsymbol{\mu}^{(1)}, \boldsymbol{\mu}^{(2)}, \ldots, \boldsymbol{\mu}^{(t)}\right)$, na qual pode ser calculada a verossimilhança marginal apresentada em (3), dada por

$$
\hat{\pi}(\boldsymbol{y} \mid M)=\frac{1}{T} \sum_{t=1}^{T} L\left(\boldsymbol{\mu}^{(t)}, M\right)
$$

em que $T$ é o tamanho da amostra obtida por simulação. 


\subsection{Modelos para dados com superdispersão}

Um conjunto de dados, às vezes, apresenta variabilidade maior do que a esperada pelos modelos probabilísticos padrões, ou seja, uma deviance residual maior do que a esperada. Esse fenômeno é conhecido como superdispersão, podendo ocorrer devido a uma variabilidade da média, excesso de zeros, correlação entre indivíduos e (ou) omissão de variáveis não observadas. Por esse motivo, existem diferentes modelos e métodos de estimação na literatura (Collett, 1991; Williams 1982, 1987; Breslow, 1984; Lawless, 1987; McCullagh \& Nelder, 1989 e Lindsey, 1995).

Hinde \& Demétrio (1998 a, b), Hinde \& Demétrio (2000) e Demétrio

\& Hinde (1997) apresentam modelos que incorporam a superdispersão e discutem os métodos de estimação dos parâmetros e técnicas para a verificação de ajuste. Essas técnicas podem ser estudadas, inicialmente, por procedimentos que se enquadrem, de uma forma geral, em dois grupos:

(i) assumir uma forma mais geral para a função de variância, possivelmente incluindo parâmetros adicionais que podem ser estimados por quaseverossimilhança (modelos de média-variância), pseudo-verossimilhança e métodos dos momentos e

(ii) assumir um modelo de dois estágios para a resposta, isto é, assumir que o parâmetro do modelo para a resposta tem alguma distribuição, levando a modelos de probabilidade compostos, como o binomial negativo, beta-binomial, Poisson-normal, logístico-normal e probit-normal podendo-se utilizar para estimação dos parâmetros o método da máxima verossimilhança ou métodos aproximados como em (i).

Supondo que a variável aleatória $Y_{i}$ representa números de sucessos em amostras de tamanhos $m_{i}$ (modelo binomial) e que essa resposta depende de covariáveis associadas ao modelo logístico, a função de variância para o modelo superdisperso pode ser representada por

$$
\operatorname{Var}\left(Y_{i}\right)=\phi m_{i} p_{i}\left(1-p_{i}\right),
$$


sendo $p_{i}$ a probabilidade de sucesso e $\phi \geq 0$ um parâmetro de escala desconhecido.

Uma função de variância geral para dados de proporção foi apresentada por Hinde \& Demétrio (1998b) dada por

$$
\operatorname{Var}\left(Y_{i}\right)=m_{i} p_{i}\left(1-p_{i}\right)\left\{1+\phi\left(m_{i}-1\right)^{\delta_{1}}\left[p_{i}\left(1-p_{i}\right)\right]^{\delta_{2}}\right\}
$$

em que $\phi, \delta_{1}$ e $\delta_{2}$ fornecem várias outras funções de variância. No caso em que $\phi=0$, retorna-se à variância do modelo binomial, dada por

$$
\operatorname{Var}\left(Y_{i}\right)=m_{i} p_{i}\left(1-p_{i}\right)
$$

Considerando um modelo de dois estágios, definido em (ii), assume-se que

$$
Y_{i} \mid P_{i} \sim \operatorname{Bin}\left(m_{i}, P_{i}\right)
$$

em que $P_{i}$ é uma variável aleatória com média e variância dada por

$$
E\left(P_{i}\right)=p_{i}
$$

$\mathrm{e}$

$$
\operatorname{Var}\left(P_{i}\right)=\phi p_{i}\left(1-p_{i}\right)
$$

Então, incondicionalmente, tem-se

$$
E\left(Y_{i}\right)=m_{i} p_{i}
$$

$\mathrm{e}$

$$
\operatorname{Var}\left(Y_{i}\right)=m_{i} p_{i}\left(1-p_{i}\right)\left[1+\phi\left(m_{i}-1\right)\right]
$$

representando o modelo 2 de Williams (Williams, 1982) que é um caso especial de (5) para $\delta_{1}=1$ e $\delta_{2}=0$. Obtém-se o mesmo resultado supondo que

$$
P_{i} \sim \operatorname{Beta}\left(a_{i}, b_{i}\right)
$$

com $\left(a_{i}+b_{i}\right)$ constante, levando ao modelo beta-binomial. 
Uma maneira conceitualmente diferente para ajustar modelos com superdispersão é assumir que a variável resposta é não independente. Considerando $Y_{i}=\sum_{j=1}^{m_{i}} R_{i j}$, assume-se distribuição Bernoulli para $R_{i j}$, ou seja,

$$
R_{i j} \sim B\left(p_{i}\right)
$$

$\operatorname{com} E\left(R_{i j}\right)=p_{i}$ e $\operatorname{Var}\left(R_{i j}\right)=p_{i}\left(1-p_{i}\right)$. Asssumindo uma correlação constante $\rho$ entre as observações, tem-se $\operatorname{Cov}\left(R_{i, j}, R_{i, k}\right)=\rho p_{i}\left(1-p_{i}\right)$, com $j \neq k$. Então,

$$
E\left(Y_{i}\right)=m_{i} p_{i}
$$

e

$$
\operatorname{Var}\left(Y_{i}\right)=m_{i} p_{i}\left(1-p_{i}\right)\left[1+\rho\left(m_{i}-1\right)\right]
$$

sendo exatamente igual à equação (6), com a vantagem de que, agora é possível estimar a subdispersão, pois $\frac{-1}{m_{i}-1}<\rho<1$.

A superdispersão pode, ainda, ser atribuída ao fato de que há variáveis explanatórias relevantes que não foram consideradas no modelo, ou devido à inclusão de algumas variáveis que não são adequadas ao modelo. Uma alternativa plausível é incluir ao preditor linear $\left(\eta_{i}\right)$, efeitos aleatórios, isto é

$$
\eta_{i}=\boldsymbol{x}_{i}^{T} \boldsymbol{\beta}+\gamma_{i}
$$

sendo $\gamma_{i}$ o efeito aleatório. Uma alternativa, para expressar esse modelo, é supor que $Z$ é uma variável aleatória com média zero e variância um, isto é,

$$
\eta_{i}=\boldsymbol{x}_{i}^{T} \boldsymbol{\beta}+\boldsymbol{\sigma} z_{i}
$$

sendo $\boldsymbol{\sigma}$, o coeficiente do termo aleatório $z_{i}$. Freqüentemente, assume-se que $Z$ tem distribuição normal padrão, isto é, $Z \sim N(0,1)$ e sua função de variância pode ser aproximada por (Hinde \& Demétrio, 1998b)

$$
\operatorname{Var}\left(Y_{i}\right) \approx m_{i} p_{i}\left(1-p_{i}\right)\left[1+\sigma^{2}\left(m_{i}-1\right) p_{i}\left(1-p_{i}\right)\right]
$$

Esse modelo é conhecido como modelo 3 de Williams (Williams, 1982). 
Como visto anteriormente, quando dados de proporção apresentam superdispersão com diferentes formas para a função de variância, faz-se necessário estimar o parâmetro $\phi$, a fim de se obterem o erro padrão de $\hat{\boldsymbol{\mu}}$ e os intervalos de confiança e testes de hipóteses para $\boldsymbol{\mu}$. Os métodos mais usados para a estimação de $\phi$ são: método da máxima verossimilhança, método dos momentos e perfil da verossimilhança.

A estimação de $\phi$ pelo método dos momentos, baseia-se no fato de que $S_{q}(\hat{\boldsymbol{\mu}}, \phi ; \boldsymbol{y}) \sim \chi_{n-q}^{2} . \log O$

$$
E\left[S_{q}(\hat{\boldsymbol{\mu}}, \phi ; \boldsymbol{y})\right]=\frac{1}{\hat{\phi}} \approx n-q
$$

com isso,

$$
\hat{\phi}=\frac{D(\hat{\boldsymbol{\mu}} ; \boldsymbol{y})}{n-q}
$$

em que $D(\hat{\boldsymbol{\mu}} ; \boldsymbol{y})$ é a deviance do modelo corrente.

Williams (1982) mostra que uma estimativa, $\hat{\phi}$, do parâmetro $\phi$ pode ser determinada substituindo a deviance, $D(\hat{\boldsymbol{\mu}} ; \boldsymbol{y})$, pela estatística $X^{2}$ generalizada de Pearson. Essa estatística, para o modelo binomial, é dada por

$$
X^{2}=\sum_{i=1}^{n} \frac{\left(y_{i}-m_{i} \hat{p}_{i}\right)^{2}}{m_{i} \hat{p}_{i}\left(1-\hat{p}_{i}\right)} .
$$

O valor estimado para o parâmetro $\phi$ dependerá das covariáveis associadas ao modelo. Entretanto, a melhor estimativa de $\phi$ é obtida usando o modelo maximal, dada por

$$
\tilde{\phi}=\frac{X^{2}}{n-k} \quad \text { ou } \quad \tilde{\tilde{\phi}}=\frac{D(\hat{\boldsymbol{\mu}} ; \boldsymbol{y})}{n-k},
$$

sendo $k$ o número de parâmetros do modelo maximal.

Considerando a função de variância dada em (4), o parâmetro $\phi$ pode ser estimado usando métodos Bayesianos. Através da distribuição conjunta a posteriori dada por

$$
\pi(\boldsymbol{\mu}, \phi ; \boldsymbol{y})=L(\boldsymbol{\mu}, \phi ; \boldsymbol{y}) \pi(\phi) \pi(\boldsymbol{\mu})
$$


a estimativa de $\phi$ pode ser obtida usando as distribuições condicionais para $\phi$ e para $\boldsymbol{\mu}$, dadas respectivamente por

$$
\pi(\phi \mid \boldsymbol{\mu}, \boldsymbol{y})=L(\boldsymbol{\mu}, \phi ; \boldsymbol{y}) \pi(\phi)
$$

$\mathrm{e}$

$$
\pi(\boldsymbol{\mu} \mid \phi, \boldsymbol{y})=L(\boldsymbol{\mu}, \phi ; \boldsymbol{y}) \pi(\boldsymbol{\mu})
$$

\subsection{Modelos para dados com excesso de zeros}

Cobrindo aspectos de ajuste e inferência, Ridout et al. (1998) apresentam uma revisão de literatura e discutem uma metodologia geral para modelar dados de contagem inflacionados de zeros, com ênfase em aplicações em Agricultura e Horticultura, enquanto que Vieira (1998) e Vieira et al. (2000) apresentam alguns modelos para dados de proporções inflacionados de zeros.

No primeiro procedimento a distribuição sob hipótese, por exemplo, a binomial é modificada para aumentar a probabilidade de uma proporção ser igual a zero. Especificamente, assume-se que a população que está sendo amostrada é composta de uma mistura de indivíduos de uma distribuição sob hipótese e de indivíduos que dão origem a zeros. Isso leva a um parâmetro adicional no modelo indicando a proporção de indivíduos do segundo tipo na população. Lambert (1992) foi a pioneira no uso de modelos de regressão para contagens com distribuição inflacionada de zeros.

O segundo procedimento modela separadamente a proporção de zeros e a média das contagens positivas. A proporção de zeros pode ser modelada através de um modelo logístico, enquanto que as contagens positivas por uma distribuição truncada (Ridout et al., 1998). Heibron (1994) considera o ajuste do modelo inflacionado de zeros em duas partes, que na econometria (Mullahy, 1986, 1997) é chamado de modelo de barreira (hurdle model).

Outras alternativas incluem modelos de barreiras semi-paramétricos e modelos de processo de nascimento (Faddy, 1997, 1998). 
Em muitos casos, para dados de contagens, não é possível observar a ocorrência de algum valor ou o pesquisador não tem interesse nesse valor, em geral, o zero. Nesse caso, considera-se que a variável aleatória $Y_{i}$ tem uma distribuição truncada no zero, portanto a função densidade de probabilidade é dada por

$$
P\left(Y_{i}=y_{i}\right)=\frac{P\left(Z_{i}=z_{i}\right)}{1-P\left(Z_{i}=0\right)}, \quad z_{i}=y_{i}=1,2, \ldots, m_{i}
$$

sendo $Z$ uma variável aleatória que tem alguma distribuição discreta (Poisson, binomial negativa e binomial). Para a distribuição binomial, tem-se

$$
P\left(Z_{i}=z_{i}\right)=\left(\begin{array}{c}
m_{i} \\
z_{i}
\end{array}\right) p_{i}^{z_{i}}\left(1-p_{i}\right)^{m_{i}-z_{i}} \quad z_{i}=0,1,2, \ldots, m_{i}
$$

e portanto, a distribuição de probabilidade de $Y_{i}$ é dada por

$$
P\left(Y_{i}=y_{i}\right)=\frac{\left(\begin{array}{c}
m_{i} \\
y_{i}
\end{array}\right) p_{i}^{y_{i}}\left(1-p_{i}\right)^{m_{i}-y_{i}}}{1-\left(1-p_{i}\right)^{m_{i}}}, \quad y_{i}=1,2, \ldots, m_{i}
$$

em que $0<p_{i} \leq 1$, com média e variância dadas por

$$
E\left(Y_{i}\right)=\frac{m_{i} p_{i}}{1-\left(1-p_{i}\right)_{i}^{m}}=\mu_{i}
$$

e

$$
\operatorname{Var}\left(Y_{i}\right)=\mu_{i}\left[1-\mu_{i}+p_{i}\left(m_{i}-1\right)\right]
$$

As probabilidades de sucesso são associadas a covariáveis através da função de ligação logística, isto é,

$$
\log \left(\frac{p_{i}}{1-p_{i}}\right)=\boldsymbol{x}_{i}^{T} \boldsymbol{\beta}
$$

em que $\boldsymbol{x}_{i}^{T}$ é a matriz do delineamento e $\boldsymbol{\beta}$ o vetor dos parâmetros.

Os modelos truncados são mais comumente encontrados na literatura, usando a distribuiçõa Poisson. Ridout \& Demétrio (1992) utilizam essa distribuição para modelar número de raízes emitidas, dado que o explante regenerou, de um experimento de cultura de tecidos.

Grogger \& Carson (1991) ajustaram um modelo Poisson truncado no zero para dados simulados da distribuição binomial negativa truncada no zero, e como resultado encontraram um viés acima de $30 \%$ para os parâmetros estimados. 
Para os casos em que existe interesse do pesquisador em observar as contagens de zero, um modelo que pode ser usado é o modelo inflacionado de zeros, proposto por Lambert (1992). A autora propôs uma mistura de distribuições Bernoulli-Poisson para modelar o excesso de zeros para a contagem de defeitos de fabricação de placas de circuito impresso. Essa metodologia, aplicada a dados de contagem, mostrou-se de grande utilidade devido ao fato de ser possível associar regressores que influenciam no parâmetro de mistura.

No caso de dados na forma de proporções, especifica-se o modelo binomial inflacionado de zeros, supondo que alguns zeros ocorrem com probabilidade $w_{i}$, enquanto que a outra parte envolvendo uma distribuição de probabilidade ocorre com probabilidade $\left(1-w_{i}\right)$. Esse modelo retorna uma distribuição de mistura de dois componentes com função de probabilidade, dada por

$$
P\left(Y_{i}=y_{i}\right)= \begin{cases}w_{i}+\left(1-w_{i}\right)\left(1-p_{i}\right)^{m_{i}}, & y_{i}=0 \\
\left(1-w_{i}\right)\left(\begin{array}{c}
m_{i} \\
y_{i}
\end{array}\right) p_{i}^{y_{i}}\left(1-p_{i}\right)^{m_{i}-y_{i}}, & y_{i}=1,2, \ldots, m_{i}\end{cases}
$$

com esperança e variância dadas respectivamente por,

$$
E\left(Y_{i}\right)=\left(1-w_{i}\right) m_{i} p_{i}=\mu_{i}
$$

e

$$
\operatorname{Var}\left(Y_{i}\right)=\mu_{i}\left[1-p_{i}+\left(\frac{w_{i}}{1-w_{i}}\right) \mu_{i}\right] .
$$

O parâmetro $w_{i}$, nesse caso, é uma probabilidade, portanto, existe a restrição de que $0 \leq w_{i}<1$. Quando $w_{i}>0$, tem-se que $\operatorname{Var}\left(Y_{i}\right)$ está sendo inflacionada, ou seja, existe superdispersão, devido ao excesso de zeros. No caso em que $w_{i}=0, \operatorname{Var}\left(Y_{i}\right)$ reduz-se à variância do modelo binomial padrão.

Para o modelo dependendo de covariáveis, faz-se necessário o uso de funções de ligação para $\boldsymbol{p}$ e para $\boldsymbol{w}$. De acordo com Vieira (1998), elas podem ser representadas por

$$
\log \left(\frac{p_{i}}{1-p_{i}}\right)=\boldsymbol{x}_{i}^{T} \boldsymbol{\beta} \quad e \quad \log \left(\frac{w_{i}}{1-w_{i}}\right)=\boldsymbol{g}_{i}^{T} \boldsymbol{\gamma},
$$

em que $\boldsymbol{x}_{i}^{T}$ e $\boldsymbol{g}_{i}^{T}$ são as matrizes de delineamento e $\boldsymbol{\beta}$ e $\boldsymbol{\gamma}$ os vetores dos parâmetros. 
Os modelos inflacionados de zeros têm sido usados para a modelagem dos dados, em áreas tais como Epidemiologia, Sociologia, Psicologia, Engenharia, Odontologia, Agricultura entre outras. Gurmu \& Trivedi (1996) aplicaram esse modelo para dados de contagem do número de viagens em navios e utilizam o método de estimação semiparamétrica para os parâmetros do modelo, proposto por Gurmu et al. ${ }^{1}$

Para os casos em que os dados de contagem são analisados longitudinalmente, espera-se que exista correlação entre as observações medidas ao longo do tempo, portanto incluem-se efeitos aleatórios ao preditor linear para incorporar essa correlação no modelo. Aplicações, usando as distribuições binomial e Poisson, para dados de horticultura, são apresentados por Hall (2000). Seguindo essa mesma metodologia, Costa (2003) usa a distribuição de Poisson para o estudo do número de lagartas em milho de proveniência transgênica e convencional. Outro exemplo é apresentado por Yan \& Lee (2001), em um estudo cujo objetivo foi observar a eficiência de um programa de prevenção de lesões em funcionários expostos a serviços de risco.

Uma distribuição de contagem, que é definida de maneira similar ao modelo inflacionado de zeros é a Poisson generalizada inflacionada de zeros, apresentada por Gupta \& Tripathi (1996). Apesar dessa distribuição não ser aplicada para dados na forma de proporção é adequada para modelar dados com superdispersão e excesso de zeros.

A função de probabilidade para a variável aleatória $Z$ com distribuição Poisson generalizada é dada por

$$
P\left(Z_{i}=z_{i}\right)=\frac{\left(1+\alpha_{i} z_{i}\right)^{z_{i}-1}}{z_{i} !} \frac{\left(\lambda_{i} e^{-\alpha_{i} \lambda_{i}}\right)_{i}^{z}}{e^{\lambda_{i}}}
$$

com $0 \leq \alpha_{i} \leq 1$ e $\lambda_{i}>0$, em que o parâmetro $\alpha_{i}$ é usado para modelar a superdispersão dos dados. A distribuição Poisson generalizada inflacionada de zeros é dada

\footnotetext{
${ }^{1}$ GURMU, S.; RILSTONE, P.; STERN, S. Semiparametric estimation of count regression models. Trabalho apresentado ao North American Summer Meetings of the Econometric Society, Quebec, 1995.
} 
por

$$
P\left(Y_{i}=y_{i}\right)= \begin{cases}w_{i}+\left(1-w_{i}\right) e^{-\lambda_{i}}, & y_{i}=0 \\ \left(1-w_{i}\right) P\left(Z_{i}=z_{i}\right), & z_{i}=y_{i}=1,2, \ldots\end{cases}
$$

Considerando o caso particular em que $w_{i}=1$, a densidade de $Y_{i}$ é dada pela distribuição Poisson generalizada. Quando $\frac{e^{\lambda_{i}}}{e^{\lambda_{i}}-1}<w_{i}<1$ tem-se o modelo deflacionado de zeros.

Para associar as covariáveis ao modelo, a função de ligação para $\boldsymbol{\lambda}$ pode ser a função logarítmica, enquanto que $\boldsymbol{w}$ pode ser dada pela segunda expressão da equação (9), para o caso do modelo inflacionado de zeros. Para $\boldsymbol{\alpha}$, a função de ligação é dada por

$$
\log \left(\frac{\alpha_{i}}{1-\alpha_{i}}\right)=\boldsymbol{w}_{i}^{T} \boldsymbol{\gamma}
$$

Um modelo alternativo em que os componentes dos dois modelos são ajustados separadamente para o subconjunto de respostas positivas, considerando uma distribuição de contagens truncada e para o vetor de respostas dicotômicas, como uma variável indicadora, foi proposto por Heilbron (1994). Entretanto, a característica de ajustar o modelo separadamente é uma conveniência que pode ser útil para seleção de modelos e para a seleção de uma distribuição de mistura. Se os parâmetros do modelo para contagens positivas e do modelo para proporção de zeros não são correlacionados, então, podem ser estimados separadamente. Esse modelo pode ser definido através das seguintes partes:

(i) seja $p_{1}\left(. \mid \mu_{1}\right)$ o modelo para respostas positivas, ou seja, um modelo truncado no zero, então

$$
P\left(Y_{i}=y_{i} \mid Y_{i}>0\right)=\frac{p_{1}\left(y_{i} \mid \mu_{1}\right)}{1-p_{1}\left(0 \mid \mu_{1}\right)}
$$

(ii) e $p_{2}\left(. \mid \mu_{2}\right)$ o modelo para a variável dicotômica.

Portanto, a distribuição de probabilidade de $Y_{i}$ é dada por

$$
P\left(Y_{i}=y_{i}\right)= \begin{cases}P\left(Y_{i}=0\right)=p_{2}\left(0 \mid \mu_{2}\right), & y_{i}=0 \\ P(Y>0)=\left(1-p_{2}\left(0 \mid \mu_{2}\right)\right) \frac{p_{1}\left(y_{i} \mid \mu_{1}\right)}{1-p_{1}\left(0 \mid \mu_{1}\right)}, & y_{i}=1,2, \ldots, m_{i} .\end{cases}
$$


Considerando o caso particular em que o modelo $p_{1}\left(y_{i} \mid \mu_{1}\right)$ segue distribuição binomial e $p_{2}\left(0 \mid \mu_{2}\right)$ é a proporção de zeros sendo representada por $w_{0}$, a distribuição de $Y_{i}$ é dada por

$$
P\left(Y_{i}=y_{i}\right)= \begin{cases}w_{0}, & y_{i}=0 \\
\left(1-w_{0}\right) \frac{\left(\begin{array}{c}
m_{i} \\
y_{i}
\end{array}\right) p_{i}^{y_{i}}\left(1-p_{i}\right)^{m_{i}-y_{i}}}{1-\left(1-p_{i}\right)_{i}^{m}}, & y_{i}=1,2, \ldots, m_{i}\end{cases}
$$

que é somente uma reparametrização do modelo binomial inflacionado de zeros, com $w_{0}=w_{i}+\left(1-w_{i}\right)\left(1-p_{i}\right)_{i}^{m}$.

Quando as mesmas covariáveis estão associadas a $\boldsymbol{w}_{\mathbf{0}}$ e a $\boldsymbol{p}$, pode-se considerar um modelo mais simples que envolve a função de ligação complemento log-log para $\left(1-\boldsymbol{w}_{\mathbf{0}}\right)$ ou $\boldsymbol{w}_{+}=\left(1-\boldsymbol{w}_{\mathbf{0}}\right)$ e uma função de ligação logística para $\boldsymbol{p}$,

$$
\log \left(\frac{p_{i}}{1-p_{i}}\right)=\boldsymbol{x}_{i}^{T} \boldsymbol{\beta} \quad \text { e } \quad \log \left[-\log \left(1-\boldsymbol{w}_{+}\right)\right]=\boldsymbol{x}_{i}^{T} \boldsymbol{\gamma}
$$

Uma extensão desse modelo foi proposto por Gurmu (1998), baseandose nas distribuições Poisson e binomial negativa. A vantagem desse modelo é a inclusão de um parâmetro que ajusta a dispersão dos dados, sendo portanto, adequado para dados com superdispersão ou subdispersão. O autor ilustrou a teoria analisando um conjunto de dados da área médica, referentes a número de visitas ao médico feitas por pacientes.

Um outro tipo de modelo, proposto por Faddy (1997), baseia-se no modelo de processo de nascimento, definido por Cox \& Miller (1965). Esse processo é uma série de eventos que ocorrem em um tempo $t$. Quando a distribuição Poisson é usada nesse processo, a probabilidade não depende do número de ocorrências dos eventos no tempo $\mathrm{t}$, sendo dada por $\lambda_{i} \delta_{i} \mathrm{t}+\mathrm{o}\left(\delta_{i} \mathrm{t}\right)$. Se $Y_{i}(t)$ é o número de eventos que ocorre no intervalo $(0, \mathrm{t})$, então o modelo é dado por

$$
P\left\{Y_{i}\left(t+\delta_{i} t\right)=y_{i}+1 \mid Y_{i}(t)=y_{i}\right\}=\lambda_{i} \delta_{i} t+o\left(\delta_{i} t\right)
$$

em que $Y_{i}(t)$ tem distribuição de Poisson com média $\delta_{i} t$.

Para outras distribuições de contagem tem-se que o processo depende 
do número de eventos, ou seja,

$$
P\left\{Y_{i}\left(t+\delta_{i} t\right)=y_{i}+1 \mid Y_{i}(t)=y_{i}\right\}=\lambda_{y_{i}} \delta_{i} t+o\left(\delta_{i} t\right)
$$

Se a sequência $\lambda_{y_{i}}$ cresce linearmente com $y_{i}$, então a distribuição adequada a utilizar é a binomial negativa. Generalizando o uso da sequência $\lambda_{y_{i}}$, tem-se que alguma distribuição de contagem pode ser obtida pela escolha adequada da sequência $\lambda_{y_{i}}$. Um caso particular dessa sequência é,

$$
\lambda_{y_{i}}= \begin{cases}\lambda_{0} & y_{i}=0 \\ \lambda_{1} & y_{i}>0\end{cases}
$$

Considerando a distribuição Poisson, quando $\lambda_{1}>\lambda_{0}$ o modelo tornase bastante útil para a identificação de excesso de zeros. Para os modelos de regressão, as covariáveis podem ser associadas a $\lambda_{0}$ e $\lambda_{1}$ por meio da função de ligação log-linear.

\subsubsection{Métodos de Estimação}

\subsubsection{Clássicos}

\section{Máxima Verossimilhança}

Os modelos inflacionados de zeros podem ter seus parâmetros estimados através do método da máxima verossimilhança.

Para escrever a função de verossimilhança, considera-se o caso particular do modelo binomial inflacionado de zeros, supondo que valores iguais a zero podem ocorrer devido a um processo binomial ou a um estado zero, sendo $B_{i}$ uma variável aleatória indicadora para o estado de cada observação, tem-se

$$
B_{i}=\left\{\begin{array} { l l } 
{ 1 : } & { \text { estado zero } } \\
{ 0 : } & { \text { estado binomial } }
\end{array} \quad \text { e } \left\{\begin{array}{l}
\left(Y_{i} \mid B_{i}=1\right) \equiv 0 \\
\left(Y_{i} \mid B_{i}=0\right) \sim \operatorname{Bin}\left(m_{i}, p_{i}\right)
\end{array}\right.\right.
$$

Considera-se que $B_{i} \sim B\left(w_{i}\right)$, isto é

$$
P\left(B_{i}=b_{i}\right)=w_{i}^{b_{i}}\left(1-w_{i}\right)^{1-b_{i}} ; \quad b_{i}=0,1
$$


e incondicionalmente $Y_{i} \sim Z I B$. Então, o logaritmo da função de verossimilhança para o modelo ZIB é dado por

$$
\begin{aligned}
l(\boldsymbol{p}, \boldsymbol{w} ; \boldsymbol{y}, \boldsymbol{b})= & \log \left[\prod_{i=1}^{n} f\left(y_{i}, b_{i}, p_{i}, w_{i}\right)\right] \\
= & \sum_{i=1}^{n}\left[b_{i} \log \left(\frac{w_{i}}{1-w_{i}}\right)+\log \left(1-w_{i}\right)\right] \\
& +\sum_{i=1}^{n}\left(1-b_{i}\right)\left[y_{i} \log \left(\frac{p_{i}}{1-p_{i}}\right)+m_{i} \log \left(1-p_{i}\right)+\log \left(\begin{array}{c}
m_{i} \\
y_{i}
\end{array}\right)\right] \\
= & l(\boldsymbol{w} \mid \boldsymbol{b})+l(\boldsymbol{p} \mid \boldsymbol{y}, \boldsymbol{b}) .
\end{aligned}
$$

A modelagem de ambos os vetores do parâmetro da binomial, $\boldsymbol{p}$, e da probabilidade do excesso de zeros, $\boldsymbol{w}$, envolve covariáveis associadas ao modelo através de

$$
\log \left(\frac{p_{i}}{1-p_{i}}\right)=\boldsymbol{x}_{i}^{T} \boldsymbol{\beta} \quad e \quad \log \left(\frac{w_{i}}{1-w_{i}}\right)=\boldsymbol{g}_{i}^{T} \boldsymbol{\gamma}
$$

sendo $\boldsymbol{\beta}$ e $\boldsymbol{\gamma}$ os vetores dos parâmetros desconhecidos e, portanto, o logaritmo da função de verossimilhança para o modelo com covariáveis é dado por $l(\boldsymbol{\beta}, \boldsymbol{\gamma} ; \boldsymbol{y}, \boldsymbol{b})$. As estimativas de máxima verossimilhança para $\boldsymbol{\beta}$ e $\boldsymbol{\gamma}$ podem ser obtidas usando a função escore ou o método de Newton-Raphson.

\section{Método escore}

A função escore é mais apropriada do que o método de NewtonRaphson, pois a derivada de segunda ordem de $l(\boldsymbol{\beta}, \boldsymbol{\gamma} ; \boldsymbol{y}, \boldsymbol{b})$ em relação a $\boldsymbol{\beta}$ e $\boldsymbol{\gamma}$ pode ser simplificada usando as esperanças das derivadas de segunda ordem (Jansakul, 2001). O vetor da função escore é dado por

$$
s(\boldsymbol{\beta}, \gamma)=\left[\begin{array}{c}
s_{\beta}(\boldsymbol{\beta}, \boldsymbol{\gamma}) \\
s_{\gamma}(\boldsymbol{\beta}, \boldsymbol{\gamma})
\end{array}\right]=\left[\begin{array}{c}
\frac{\partial l(\boldsymbol{\beta}, \boldsymbol{\gamma} ; \boldsymbol{y}, \boldsymbol{b})}{\partial \boldsymbol{\beta}} \\
\frac{\partial l(\boldsymbol{\beta}, \boldsymbol{\gamma} ; \boldsymbol{y}, \boldsymbol{b})}{\partial \boldsymbol{\gamma}}
\end{array}\right]
$$

em que

$$
\frac{\partial l(\boldsymbol{\beta}, \boldsymbol{\gamma} ; \boldsymbol{y}, \boldsymbol{b})}{\partial \boldsymbol{\beta}_{j}}=\sum_{i=1}^{n}\left\{b_{i}\left[\frac{-\left(1-w_{i}\right)\left(1-p_{i}\right)^{m_{i}}}{w_{i}+\left(1-w_{i}\right)\left(1-p_{i}\right)^{m_{i}}}\right] m_{i} p_{i}+\left(1-b_{i}\right)\left(y_{i}-m_{i} p_{i}\right)\right\} x_{i j}
$$


e

$$
\frac{\partial l(\boldsymbol{\beta}, \boldsymbol{\gamma} ; \boldsymbol{y}, \boldsymbol{b})}{\partial \boldsymbol{\gamma}_{r}}=\sum_{i=1}^{n}\left\{b_{i}\left[\frac{w_{i}}{w_{i}+\left(1-w_{i}\right)\left(1-p_{i}\right)^{m_{i}}}\right]-\left(1-b_{i}\right) w_{i}\right\} g_{i r}
$$

A matriz informação de Fisher, $I(\boldsymbol{\beta}, \boldsymbol{\gamma})$, pode ser subdividida em

$$
I(\boldsymbol{\beta}, \boldsymbol{\gamma})=\left[\begin{array}{cc}
I_{\beta \beta}(\boldsymbol{\beta}, \boldsymbol{\gamma}) & I_{\beta \gamma}(\boldsymbol{\beta}, \boldsymbol{\gamma}) \\
I_{\gamma \beta}(\boldsymbol{\beta}, \boldsymbol{\gamma}) & I \gamma \gamma(\boldsymbol{\beta}, \boldsymbol{\gamma})
\end{array}\right]
$$

que é simétrica e cujos componentes são dados por

$$
\begin{gathered}
I_{\beta \beta}(\boldsymbol{\beta}, \boldsymbol{\gamma})=-E\left[\frac{\partial^{2} l(\boldsymbol{\beta}, \boldsymbol{\gamma} ; \boldsymbol{y}, \boldsymbol{b})}{\partial \boldsymbol{\beta} \partial \boldsymbol{\beta}^{\prime}}\right], I_{\beta \gamma}(\boldsymbol{\beta}, \boldsymbol{\gamma})=-E\left[\frac{\partial^{2} l(\boldsymbol{\beta}, \boldsymbol{\gamma} ; \boldsymbol{y}, \boldsymbol{b})}{\partial \boldsymbol{\beta} \partial \boldsymbol{\gamma}}\right], \\
I_{\gamma \gamma}(\boldsymbol{\beta}, \boldsymbol{\gamma})=-E\left[\frac{\partial^{2} l(\boldsymbol{\beta}, \boldsymbol{\gamma} ; \boldsymbol{y}, \boldsymbol{b})}{\partial \boldsymbol{\gamma} \partial \boldsymbol{\gamma}^{\prime}}\right]
\end{gathered}
$$

sendo

$$
\begin{aligned}
\frac{\partial^{2} l(\boldsymbol{\beta}, \boldsymbol{\gamma} ; \boldsymbol{y}, \boldsymbol{b})}{\partial \boldsymbol{\beta}_{j} \partial \boldsymbol{\beta}_{k}}= & \sum_{i=1}^{n}\left\{b_{i}\left[\frac{\left(1-p_{i}\right)^{m_{i}}\left[\left(1-m_{i} y_{i}\right) w_{i}+\left(1-w_{i}\right)\left(1-p_{i}\right)^{m_{i}}\right]\left(1-w_{i} m_{i} p_{i}\right)}{\left[w_{i}+\left(1-w_{i}\right)\left(1-p_{i}\right)^{m_{i}}\right]^{2}}\right]\right. \\
+ & \left.\left(1-b_{i}\right)\left(m_{i} p_{i}\right)\right\} x_{i j} x_{i k}, \\
\frac{\partial^{2} l(\boldsymbol{\beta}, \boldsymbol{\gamma} ; \boldsymbol{y}, \boldsymbol{b})}{\partial \boldsymbol{\gamma}_{r} \partial \boldsymbol{\gamma}_{s}} & =\sum_{i=1}^{n}\left\{b_{i}\left[\frac{\left(1-p_{i}\right)^{m_{i}} w_{i}\left(1-w_{i}\right)}{\left[w_{i}+\left(1-w_{i}\right)\left(1-p_{i}\right)^{m_{i}}\right]^{2}}\right]-w_{i}\left(1-w_{i}\right)\right\} g_{i r} g_{i s}
\end{aligned}
$$

e

$$
\frac{\partial^{2} l(\boldsymbol{\beta}, \boldsymbol{\gamma} ; \boldsymbol{y}, \boldsymbol{b})}{\partial \boldsymbol{\beta}_{j} \partial \boldsymbol{\gamma}_{r}}=\sum_{i=1}^{n}\left\{b_{i}\left[\frac{\left(1-p_{i}\right)^{m_{i}} m_{i} p_{i} w_{i}\left(1-w_{i}\right)}{\left[w_{i}+\left(1-w_{i}\right)\left(1-p_{i}\right)^{m_{i}}\right]^{2}}\right]\right\} x_{i j} g_{i r}
$$

Utilizando o fato de que,

$$
E\left[b_{i}\right]=w_{i}+\left(1-w_{i}\right)\left(1-p_{i}\right)^{m_{i}}
$$

e

$$
E\left[\left(1-b_{i}\right)\right]=\left(1-w_{i}\right)\left(1-\left(1-p_{i}\right)^{m_{i}}\right),
$$

tem-se que

$$
\begin{aligned}
-E\left[\frac{\partial^{2} l(\boldsymbol{\beta}, \boldsymbol{\gamma} ; \boldsymbol{y}, \boldsymbol{b})}{\partial \boldsymbol{\beta}_{j} \partial \boldsymbol{\beta}_{k}}\right] & =\sum_{i=1}^{n}\left\{\frac{-\left(1-p_{i}\right)^{m_{i}}\left[\left(1-m_{i} p_{i}\right) w_{i}+\left(1-w_{i}\right)\left(1-p_{i}\right)^{m_{i}}\right]\left(1-w_{i} m_{i} p_{i}\right)}{\left[w_{i}+\left(1-w_{i}\right)\left(1-p_{i}\right)^{m_{i}}\right]}\right. \\
& \left.+m_{i} p_{i}\left(1-w_{i}\right)\left(1-\left(1-p_{i}\right)^{m_{i}}\right)\right\} x_{i j} x_{i k},
\end{aligned}
$$




$$
-E\left[\frac{\partial^{2} l(\boldsymbol{\beta}, \boldsymbol{\gamma} ; \boldsymbol{y}, \boldsymbol{b})}{\partial \boldsymbol{\gamma}_{r} \partial \boldsymbol{\gamma}_{s}}\right]=\sum_{i=1}^{n} w_{i}\left(1-w_{i}\right)\left\{1-\frac{\left(1-p_{i}\right)^{m_{i}}}{w_{i}+\left(1-w_{i}\right)\left(1-p_{i}\right)^{m_{i}}}\right\} g_{i r} g_{i s}
$$

$\mathrm{e}$

$$
-E\left[\frac{\partial^{2} l(\boldsymbol{\beta}, \boldsymbol{\gamma} ; \boldsymbol{y}, \boldsymbol{b})}{\partial \boldsymbol{\beta}_{j} \partial \boldsymbol{\gamma}_{r}}\right]=-\sum_{i=1}^{n}\left\{\frac{\left(1-p_{i}\right)^{m_{i}} w_{i}\left(1-w_{i}\right) m_{i} p_{i}}{w_{i}+\left(1-w_{i}\right)\left(1-p_{i}\right)^{m_{i}}}\right\} x_{i j} g_{i s} .
$$

Consequentemente, as estimativas de $\boldsymbol{\beta}$ e $\boldsymbol{\gamma}$ são obtidas iterativamente através de

$$
\left[\begin{array}{l}
\boldsymbol{\beta}^{(t+1)} \\
\boldsymbol{\gamma}^{(t+1)}
\end{array}\right]=\left[\begin{array}{l}
\boldsymbol{\beta}^{(t)} \\
\boldsymbol{\gamma}^{(t)}
\end{array}\right]+\left[I\left(\boldsymbol{\beta}^{(t)}, \boldsymbol{\gamma}^{(t)}\right)\right]^{-1} s\left(\boldsymbol{\beta}^{(t)}, \boldsymbol{\gamma}^{(t)}\right)
$$

em que $t$ é o número de iterações.

Com estimativas iniciais para $\boldsymbol{\beta}$ e $\boldsymbol{\gamma}$ próximas do valor de convergência, o processo iterativo convergirá rapidamente, usando algum critério de parada, tal como $\left|l(\boldsymbol{\beta}, \boldsymbol{\gamma} ; \boldsymbol{y}, \boldsymbol{b})^{(t+1)}-l(\boldsymbol{\beta}, \boldsymbol{\gamma} ; \boldsymbol{y}, \boldsymbol{b})^{(t)}\right| \leq \varepsilon$, sendo que, em geral, assume-se o valor $10^{-6}$ para $\varepsilon$. Os erros padrões de $\hat{\boldsymbol{\beta}}$ e de $\hat{\boldsymbol{\gamma}}$ são obtidos diretamente de $\left[I\left(\boldsymbol{\beta}^{(\boldsymbol{t})}, \boldsymbol{\gamma}^{(\boldsymbol{t})}\right)\right]^{-1}$ no final das iterações.

De acordo com Lambert (1992) e Meilijson (1989), para valores iniciais adequados, esses métodos convergem mais rapidamente do que o algoritmo EM, com a vantagem de fornecer estimativas para a matriz de covariância dos parâmetros. Entretanto, valores iniciais que estejam próximos da região de convergência nem sempre é simples de serem obtidos para o modelo ZIB, principalmente quando existem muitos parâmetros.

\section{Algoritmo EM}

O algoritmo EM, desenvolvido por Dempster et al. (1977), pode ser implementado usando programas tais como GLIM, S-Plus ou SAS, com a vantagem de que os valores iniciais não influenciam diretamente no procedimento da análise. Esse algoritmo gera estimativas consistentes de máxima verossimilhança que são as mesmas obtidas pelo método de Newton-Raphson. Hall (2000) fornece detalhes da aplicação do algoritmo EM para ajustar o modelo ZIB com covariáveis. 
Esse algoritmo pode ser usado para maximizar $l(\boldsymbol{p}, \boldsymbol{w} ; \boldsymbol{y}, \boldsymbol{b})$, dado em (12), alternando entre o passo E, no qual os dados não observados, $\boldsymbol{b}$, são estimados através da esperança de $(\boldsymbol{p}, \boldsymbol{w})$ e o passo M, em que a estimativa $\boldsymbol{b}$ fixada é maximizada em relação a $\boldsymbol{p}$ e $\boldsymbol{w}$.

A última expressão de (12) mostra, no entanto, que a estimação de $\boldsymbol{p}$ e $\boldsymbol{w}$ pode ser independentemente obtida, maximizando-se os dois termos de $l(\boldsymbol{p}, \boldsymbol{w} ; \boldsymbol{y}, \boldsymbol{b})$ separadamente.

Para aplicar o algoritmo EM é necessário atribuir valores iniciais $\left(\boldsymbol{p}^{(0)}, \boldsymbol{w}^{(0)}\right)$ e proceder iterativamente. A iteração $(t+1)$ é realizada através da esperança condicional $b_{i}$ e da maximização para $\boldsymbol{p}$ e $\boldsymbol{w}$.

Passos do algoritmo EM:

Passo E: Estimar $b_{i}$ da média condicional $b_{i}^{(t)}=E\left(b_{i} \mid y_{i}, \boldsymbol{w}^{(t)}, \boldsymbol{p}^{(t)}\right)$, com as estimativas dos parâmetros de regressão. Essa esperança é determinada através do teorema de Bayes, obtendo-se

$$
\begin{aligned}
& \quad b_{i}^{(t)}=P\left(B_{i}=0 \mid y_{i}, \boldsymbol{w}^{(t)}, \boldsymbol{p}^{(t)}\right) \\
& \quad=\frac{P\left(Y_{i}=y_{i} \mid B_{i}=0, \boldsymbol{p}, \boldsymbol{w}\right) P\left(B_{i}=0\right)}{P\left(Y_{i}=y_{i} \mid B_{i}=0, \boldsymbol{p}, \boldsymbol{w}\right) P\left(B_{i}=0\right)+P\left(Y_{i}=y_{i} \mid B_{i}=1, \boldsymbol{p}, \boldsymbol{w}\right) P\left(B_{i}=1\right)}, \\
& \text { e, então, }
\end{aligned}
$$

$$
E\left(B_{i} \mid Y_{i}=y_{i}, \boldsymbol{p}, \boldsymbol{w}\right)=\left\{\begin{array}{ccc}
\frac{(1-\boldsymbol{p})^{m_{i}}(1-\boldsymbol{w})}{(1-\boldsymbol{p})^{m_{i}}(1-\boldsymbol{w})+\boldsymbol{w}} & \text { se } & y_{i}=0 \\
0 & \text { se } & y_{i}>0
\end{array}\right.
$$

Passo M para $\boldsymbol{w}$ : Deve-se maximizar o logaritmo da função de verossimilhança para o parâmetro $\boldsymbol{w}$, considerando o vetor estimado $b_{i}$ obtido no passo E. Como a verossimilhança foi dividida em dois termos, pode-se maximizar apenas o termo $l(\boldsymbol{w} \mid \boldsymbol{b})$. O termo $\boldsymbol{w}$ pode ser obtido considerando como preditor linear a média geral e $b_{i}$ como variável dependente, com distribuição binomial e denominador 1.

Passo M para $\boldsymbol{p}$ : O vetor $\boldsymbol{p}$ pode ser obtido maximizando $l(\boldsymbol{p} \mid \boldsymbol{y}, \boldsymbol{b})$, que é o mesmo que maximizar o logaritmo da função de verossimilhança para um modelo 
binomial considerando $b_{i}$ como peso a priori, $y_{i}$ como variável resposta e um preditor linear.

\subsubsection{Bayesianos}

Outra maneira para a estimação dos parâmetros dos modelos para dados na forma de proporções é através da utilização de métodos Bayesianos que permitem incorporar ao modelo informações prévias a respeito dos parâmetros de interesse para a análise, o que não é feito usando-se os métodos tradicionais.

Seja $\boldsymbol{y}$ o vetor das observações e $\boldsymbol{\mu} \in \Re^{n}$ o vetor de parâmetros. Os métodos Bayesianos consideram $\boldsymbol{\mu}$ um vetor aleatório, enquanto que os métodos clássicos consideram $\boldsymbol{\mu}$ fixo e desconhecido. Na inferência Bayesiana toda informação relacionada ao parâmetro $\boldsymbol{\mu}$ pode ser obtida de uma distribuição a posteriori, $\pi(\boldsymbol{\mu} \mid$ $\boldsymbol{y})$, definida por

$$
\pi(\boldsymbol{\mu} \mid \boldsymbol{y})=\frac{L(\boldsymbol{\mu} ; \boldsymbol{y}) \pi(\boldsymbol{\mu})}{\int L(\boldsymbol{\mu} ; \boldsymbol{y}) \pi(\boldsymbol{\mu}) d \boldsymbol{\mu}}
$$

em que $\pi(\boldsymbol{\mu})$ é a distribuição a priori de $\boldsymbol{\mu}, L(\boldsymbol{\mu} ; \boldsymbol{y})$ é a função de verossimilhança em relação a $\boldsymbol{\mu}$ e $\boldsymbol{y}$ e $\int L(\boldsymbol{\mu} ; \boldsymbol{y}) \pi(\boldsymbol{\mu}) d \boldsymbol{\mu}$ é uma constante normalizadora não tendo influência sobre a estimação dos parâmetros. Portanto, a distribuição a posteriori pode ser expressa como

$$
\pi(\boldsymbol{\mu} \mid \boldsymbol{y}) \propto L(\boldsymbol{\mu} ; \boldsymbol{y}) \pi(\boldsymbol{\mu})
$$

Algumas características dessa distribuição, tais como, momentos e quantis são necessárias e podem ser obtidas através da esperança a posteriori de uma função $g(\mu)$, isto é, usando-se

$$
E[g(\boldsymbol{\mu}) \mid \boldsymbol{y}] \propto \int g(\boldsymbol{\mu}) \pi(\boldsymbol{\mu} \mid \boldsymbol{y}) d \boldsymbol{\mu} .
$$

Entretanto, normalmente, a dimensão de $\boldsymbol{\mu}$ é grande tornando a integral em (13) complicada, e portanto, a aproximação de Laplace (Kass et al. 1989) e os métodos MCMC ("Markov chain Monte Carlo") podem ser usados. 
O método de Laplace é baseado na distribuição normal. Portanto, o integrando da expressão (13) precisa ser expresso na forma $\exp \{\log [g(\boldsymbol{\mu}) \pi(\boldsymbol{\mu} \mid \boldsymbol{y})]\}$ e, expandido para $\log [g(\boldsymbol{\mu}) \pi(\boldsymbol{\mu} \mid \boldsymbol{y})]$ como função de $\boldsymbol{\mu}$, utilizando essa expressão como uma aproximação de Taylor até o segundo grau, em torno da moda. Com isso, a aproximação resultante para $g(\boldsymbol{\mu}) \pi(\boldsymbol{\mu} \mid \boldsymbol{y})$ é proporcional à densidade da distribuição normal multivariada para $\boldsymbol{\mu}$, e então,

$$
E[g(\boldsymbol{\mu}) \mid \boldsymbol{y}] \approx g\left(\boldsymbol{\mu}_{0}\right) \pi\left(\boldsymbol{\mu}_{0} \mid \boldsymbol{Y}\right)(2 \pi)^{\frac{d}{2}}\left|-u^{\prime \prime}\left(\boldsymbol{\mu}_{0}\right)\right|^{\frac{1}{2}}
$$

sendo $d$ a dimensão de $\boldsymbol{\mu}, u(\boldsymbol{\mu})=\log [g(\boldsymbol{\mu}) \pi(\boldsymbol{\mu} \mid \boldsymbol{y})], u^{\prime \prime}\left(\boldsymbol{\mu}_{0}\right)$ a derivada de segunda ordem em relação a $\boldsymbol{\mu}$ e $\boldsymbol{\mu}_{0}$ o valor em que $u(\boldsymbol{\mu})$ é maximizado.

Para os casos em que $\pi(\boldsymbol{\mu} \mid \boldsymbol{y})$ não segue a distribuição normal a aproximação de Laplace torna-se complicada, e uma alternativa é usar o método MCMC (Gelman et al., 2003).

Método MCMC

Simulação de cadeias de Markov (MCMC) é um método geral baseado na obtenção de uma amostra de valores de $\boldsymbol{\mu}$, a partir de distribuições aproximadas, que é corrigida para melhor aproximar a distribuição a posteriori $\pi(\boldsymbol{\mu} \mid \boldsymbol{y})$. As amostras são obtidas seqüencialmente, com a distribuição dos valores dependendo apenas do último valor amostrado e, portanto, formando uma cadeia de Markov.

Essa simulação é utilizada somente quando não for possível amostrar $\boldsymbol{\mu}$ diretamente de $\pi(\boldsymbol{\mu} \mid \boldsymbol{y})$, com isso cria-se um processo de Markov em que a distribuição estacionária é $\pi(\boldsymbol{\mu} \mid \boldsymbol{y})$ e obtém-se um número suficiente de pontos, tal que o processo converge se esses pontos estão próximos de um valor constante.

O sucesso do método, entretanto, não está na propriedade de Markov mas sim nas distribuições aproximadas que melhoram a cada passo da simulação, no sentido de convergir para a distribuição de interesse. Para realizar esse processo é necessário usar algum algoritmo, tal como o amostrador de Gibbs (Gelfand \& 
Smith, 1990) ou o Metropolis-Hastings (Chib \& Greenberg, 1995).

Amostrador de Gibbs

O amostrador de Gibbs é um processo iterativo, no qual se utilizam subvetores de $\boldsymbol{\mu}$ para a estimação dos parâmetros, supondo que o vetor dos parâmetros é dividido em $(q+1)$ componentes ou subvetores, $\boldsymbol{\mu}=\left(\mu_{0}, \mu_{1}, \ldots, \mu_{q}\right)^{T}$. Através do processo iterativo são gerados novos valores para os subvetores de $\boldsymbol{\mu}$, sendo que cada subvetor é condicional a todos os outros subvetores, tendo assim $(q+1)$ passos em $t$ iterações. Para a estimação de cada ponto a cada iteração, $\mu_{i}^{t}$ é amostrado usando a distribuição condicional de $\mu_{i}$ dados todos os outros componentes de $\boldsymbol{\mu}$, isto é, usando-se $\pi\left(\mu_{i}^{t} \mid \boldsymbol{\mu}_{-i}^{t-1}, \boldsymbol{y}\right)$, sendo $\boldsymbol{\mu}_{-i}^{t-1}=\left(\mu_{0}^{t}, \ldots, \mu_{i-1}^{t}, \mu_{i+1}^{t-1}, \ldots, \mu_{q}^{t-1}\right)$.

Para os casos em que as distribuições condicionais a posteriori $\pi\left(\mu_{i} \mid\right.$ $\left.\boldsymbol{\mu}_{-i}\right)$ em que $\boldsymbol{\mu}_{-i}=\left(\mu_{0}, \mu_{1}, \ldots, \mu_{i-1}, \mu_{i+1}, \ldots, \mu_{q}\right)$, não apresentam formas de distribuições conhecidas, como por exemplo normal, binomial, entre outras, o amostrador de Gibbs não pode ser utilizado, e então um algoritmo alternativo para gerar amostras de $\mu_{i}$ é o Metropolis-Hastings.

\section{Algoritmo Metropolis-Hastings}

A idéia desse algoritmo é escrever a distribuição condicional a posteriori através do produto de duas funções, isto é,

$$
\pi\left(\mu_{i} \mid \mu_{0}, \mu_{1}, \ldots, \mu_{i-1}, \mu_{i+1}, \ldots, \mu_{q}\right) \propto \Psi\left(\mu_{i}\right) q\left(\mu_{i}\right)
$$

sendo que $q\left(\mu_{i}\right)$ tem a forma de uma distribuição conhecida para que através dela sejam gerados valores de $\mu_{i}$. A função $\Psi\left(\mu_{i}\right)$ é utilizada com a função $q\left(\mu_{i}\right)$ no teste de aceitação e rejeição dos valores gerados. O algoritmo Metropolis-Hastings leva em consideração as seguintes etapas:

(i) simulação de amostras $\mu_{i}$ através de $q\left(\mu_{i}\right)$; 
(ii) cálculo da probabilidade de aceitação do novo valor

$$
\alpha\left(\mu_{i}^{t}\right)=\min \left(1, \frac{\Psi\left(\mu_{i}^{t}\right)}{\Psi\left(\mu_{i}^{t-1}\right)}\right) ;
$$

(iii) geração de um valor $U$ a partir de uma distribuição Uniforme $(0,1)$;

(iv) aceitação do ponto candidato $\mu_{i}^{t}$ se $U \leq \alpha\left(\mu_{i}^{t}\right)$ ou rejeição do mesmo em caso contrário;

(v) retorno ao passo (i), até que a convergência seja alcançada, de acordo com algum critério estabelecido.

Alguns dos critérios para verificar a convergência de $\boldsymbol{\mu}$ é observar se foram aceitos de $30 \%$ a $60 \%$ dos valores gerados, se o traço (gráfico dos valores gerados) da distribuição a posteriori condicional se aproxima de um valor constante ao longo das iterações e usando os testes de diagnóstico de Raftery \& Lewis e Heidelberger \& Welch.

No método de Raftery \& Lewis (1992) determina-se um número mínimo de iterações necessárias para que as amostras da cadeia sejam independentes e a convergência alcançada. O número de iterações sugerido pelo método é baseado no valor da variância da distribuição binomial e a estatística do teste é dada por $I=\frac{N}{N_{\min }}$, sendo $N$ o número de iterações e $N_{\text {min }}$ o número de iterações sugerido pelo método. Se o valor de $I$ for próximo de 1 a cadeia converge. Segundo os autores se $I>5$ provavelmente a cadeia apresenta problemas de convergência.

Utilizando o método de Heidelberger \& Welch (1983) a convergência da cadeia é verificada através de dois testes simultaneamente. O primeiro teste usa a estatística de Cramer-von-Mises, a qual verifica a estacionaridade da cadeia. Se a cadeia for estacionária, calcula-se um intervalo de confiança de $95 \%$ de probabilidade para o valor médio da cadeia, verificando-se que a convergência ocorre para os casos em que a amplitude entre o valor médio da cadeia e o limite superior do intervalo de confiança for menor do que 0,1 . 
Considerando que a convergência foi satisfeita, as estimativas e os desvios padrões de $\boldsymbol{\mu}$ são obtidos através da média e do desvio padrão dos valores gerados que foram aceitos no algoritmo. Os intervalos de credibilidade são obtidos através dos percentis da amostra ordenada.

\subsection{Diagnósticos para modelos lineares generalizados}

Técnicas de diagnósticos são usadas para verificar se um determinado modelo é apropriado para os dados. Essas técnicas podem ser usadas para detectar uma falha sistemática do modelo ou falhas particulares como, por exemplo, presença de outliers. Uma análise fácil para verificar a qualidade do ajuste é observar se o valor da deviance residual está próximo do número de graus de liberdade do resíduo e quando isso ocorre, significa que o modelo está bem ajustado aos dados. Outras técnicas padrões para detectar a falta de ajuste dos modelos são discutidas, por exemplo, em McCullagh \& Nelder (1989) e Collett (1991).

Para verificar a adequação do preditor linear, da função de ligação e identificar outliers são usados gráficos para os resíduos padronizados. Para checar a especificação da função de variância $V(\mu)$, utiliza-se um gráfico com os valores ajustados que, entretanto, pode não ser útil para modelos com superdispersão, pois envolve o parâmetro de escala $\phi$. Se esse parâmetro for estimado, os valores da deviance residual ou da estatística $X^{2}$ generalizada de Pearson são obrigatoriamente próximos de 1, tornando-se essas estatísticas ineficientes para verificar a qualidade do ajuste do modelo.

Um método alternativo para verificar a adequação do modelo binomial foi proposto por Liang \& McCullagh (1993), que é baseado no uso de resíduos considerando o tamanho amostral. Verificou-se, entretanto, que os gráficos obtidos por esse método não são eficientes. Ganio \& Schafer (1992), por outro lado, consideram diagnósticos para modelos com superdispersão usando uma forma de gráfico da variável adicionada para comparar as funções de variâncias. No entanto, uma técnica 
que é bastante utilizada em modelos com superdispersão e excesso de zeros é o gráfico meio normal (half normal plot) com envelope simulado. Essa técnica, originalmente proposta por Atkinson (1985), é uma modificação do gráfico Q-Q plot padrão, usando valores absolutos dos resíduos do modelo com envelope simulado, tendo a utilidade de diminuir a subjetividade da análise, detectando falhas sistemáticas no modelo e também valores atípicos.

Segundo Atkinson (1985), o modelo adequado apresenta um padrão em que os pontos se distribuem aproximadamente em torno de uma reta. O procedimento para a construção desse gráfico é dado pelas seguintes etapas:

(i) ajustar um modelo e calcular os valores absolutos ordenados de alguma medida de diagnóstico adequada;

(ii) extrair amostras aleatórias simples com reposição de tamanho $n$ para a variável resposta usando como parâmetro as estimativas obtidas pelo modelo ajustado em (i);

(iii) ajustar o mesmo modelo usando as mesmas covariáveis para cada amostra simulada e calcular os valores absolutos ordenados do diagnóstico de interesse, $d_{(j) i}^{*}, j=1, \cdots, n, i=1, \cdots, m$;

(iv) obter para cada $i$, os valores mínimo, máximo e a média do diagnóstico de interesse $d_{(j) i}^{*} \mathrm{e}$

(v) fazer o gráfico desses valores, $d_{(j) i}^{*}$, e dos observados, em que os valores mínimo e máximo são os limites inferior e superior do intervalo.

O mínimo e o máximo de $d_{(j) i}^{*}$ geram um envelope, o qual pode ser usado para verificar se os valores observados são consistentes com o modelo ajustado. Na literatura, sugere-se o uso do resíduo de Pearson padronizado ou a deviance residual padronizada, como medida de diagnóstico. Considerando um modelo geral, o resíduo de Pearson padronizado é dado por

$$
r_{P_{i}}=\frac{y_{i}-\hat{\mu}_{i}}{\sqrt{V\left(\hat{\mu}_{i}\right)\left(1-h_{i i}\right)}}
$$


em que $h_{i i}$ é o i-ésimo elemento da diagonal da matriz estimada $H=\hat{W}^{\frac{1}{2}} X\left(X^{\prime} \hat{W} X\right)^{-1} X^{\prime} \hat{W}^{\frac{1}{2}}$, sendo $W$ a matriz diagonal ponderada e $X$ a matriz de delineamento. Outra medida de diagnóstico é a deviance residual padronizada que é dada por

$$
r_{D_{i}}=\frac{\operatorname{sinal}\left(y_{i}-\hat{\mu}_{i}\right)\left\{\frac{2 w_{i}}{\phi}\left[y_{i}\left(\tilde{y}_{i}-\hat{\mu}_{i}\right)+b\left(\tilde{y}_{i}\right)-b\left(\hat{\mu}_{i}\right)\right]\right\}^{\frac{1}{2}}}{\sqrt{\hat{\phi}\left(1-h_{i i}\right)}}
$$

sendo $\tilde{y}_{i}$ e $\hat{\mu}_{i}$ as estimativas do parâmetro canônico sob os modelos saturados e corrente, respectivamente.

Demétrio \& Hinde (1997) desenvolveram uma macro para obter o gráfico meio normal com envelope simulado usando o programa GLIM, no ajuste dos modelos binomial, Poisson e para suas versões superdispersas. Uma outra macro é proposta por Friendly (2000), usando o "Procedure Genmod" do SAS para o ajuste de modelos com qualquer distribuição pertencente à família exponencial na forma canônica e para as versões com superdispersão constante dos modelos discretos e, Paula (2002) apresenta macros do envelope simulado usando o S-Plus ou o R, sendo que o autor utiliza o gráfico normal (normal plot) com envelope simulado. Para os outros modelos, como, por exemplo, os inflacionados de zeros, é necessária a construção de macros. 


\section{MATERIAL E MÉTODOS}

\subsection{Material}

Em geral, ensaios de controle biológico são conduzidos com a finalidade de identificar as condições ideais para a produção de um grande número de ovos parasitados, que serão utilizados em liberações inundativas no campo.

Os dados a serem utilizados são provenientes de um experimento realizado no Laboratório de Biologia do Departamento de Entomologia da ESALQ/USP, pela $\operatorname{Dr}^{a}$ Marta Rossi, sob coordenação do Dr. José Roberto P. Parra, cujo objetivo foi estudar o processo de competição de fêmeas de T. galloi (parasita) parasitando ovos de A. kuehniella, hospedeiro alternativo (mais econômico) a D. saccharalis, praga da cana-de-açúcar.

Os ensaios foram conduzidos em estufas sob a temperatura de $25 \pm 1^{\circ} \mathrm{C}$, umidade relativa do ar de $70 \pm 10 \%$ e fotofase de $16: 8$ (16 horas com luz e 8 horas sem luz).

As fêmeas do parasita com menos de 24 horas de idade foram isoladas em tubos de vidro de $1 \mathrm{~cm}$ de diâmetro por $7 \mathrm{~cm}$ de comprimento, tampados com algodão, tendo sido os parasitóides alimentados com mel puro. Os ovos do hospedeiro alternativo, A. kuehniella, tinham menos de 24 horas de idade e foram esterelizados em lâmpada germicida por 45 minutos.

Diferentes números $(2,4,8,16,32,64$ e 128) de fêmeas de T. galloi foram colocados em tubos de ensaio com 128 ovos de A. kuehniella de acordo com um delineamento inteiramente casualizado com 10 repetições. Os parasitóides foram deixados 24 horas e, então, retirados. Após 6 dias, foi contado o número de ovos 
de A. kuehniella escurecidos (indicativo de que o ovo do hospedeiro foi parasitado). O objetivo desse experimento foi estudar o comportamento da proporção de ovos parasitados em relação ao número de fêmeas (parasitas) e determinar o número (ideal) de fêmeas que dá a maior proporção de ovos parasitados.

Uma análise exploratória dos dados, através de um diagrama de dispersão da proporção de ovos parasitados de A. kuehniella em relação ao número de fêmeas (ver Figura 1) de T. galloi mostra que existe uma grande variabilidade dos dados e um excesso de zeros. Esses aspectos, portanto, devem ser levados em consideração nos modelos a serem utilizados na análise dos dados.

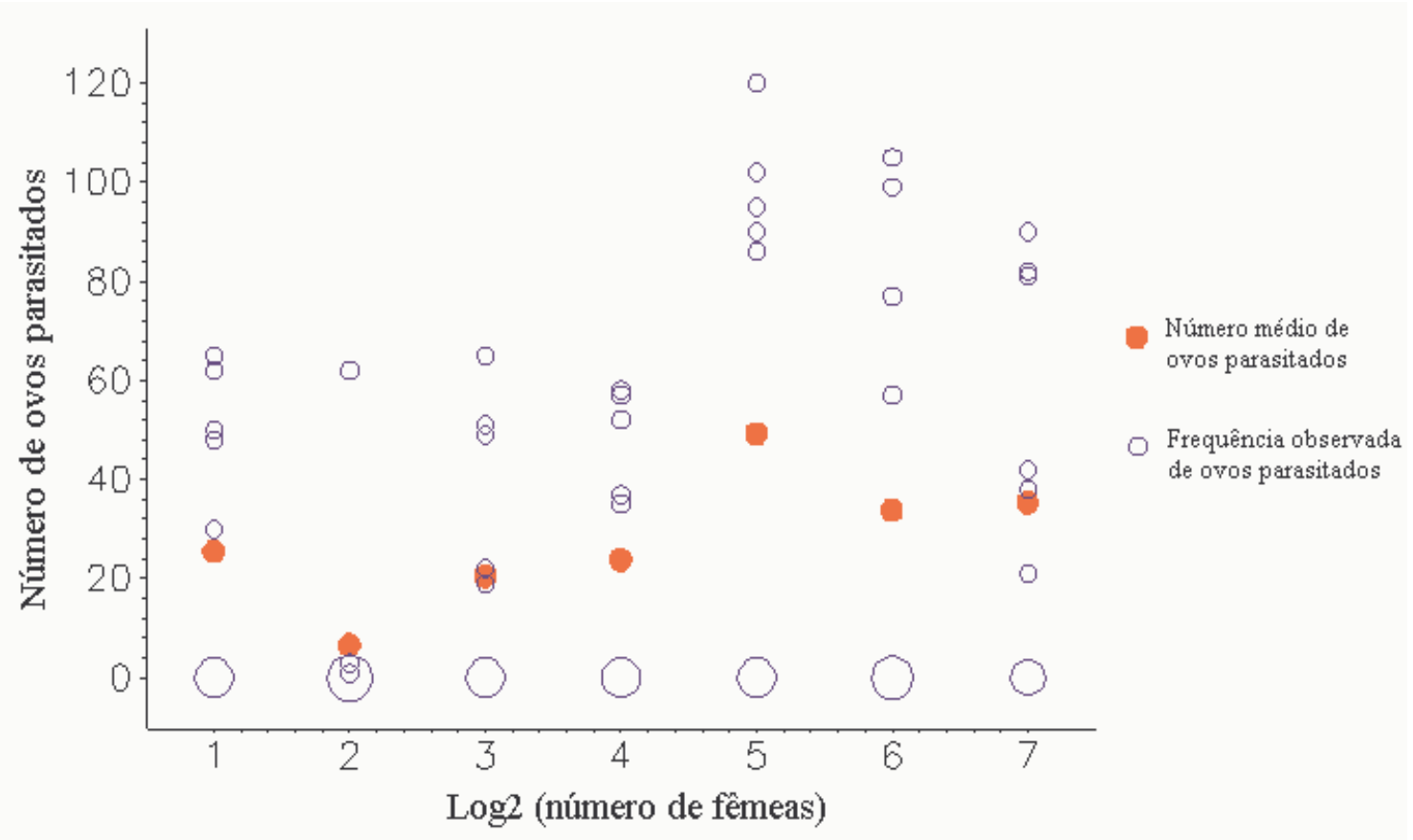

Figura 1 - Proporção de ovos de A. kuehniella parasitados, em função do número de fêmeas do T. galloi (o diâmetro do ponto reflete a grandeza da frequência) 


\subsection{Métodos}

Em um contexto geral, considere $Y_{i}$ como a variável aleatória "número

de ovos parasitados" em $m_{i}$ ovos, com observações $\left(y_{i}, m_{i}\right)$ e $p_{i}=\frac{y_{i}}{m_{i}}$ representando a proporção de ovos parasitados, $i=1,2, \ldots, 70$, sendo 70 o número total de observações no experimento. Assume-se inicialmente um modelo em que a variável $Y_{i}$ tem distribuição binomial, o preditor linear envolve um delineamento inteiramente casualizado com 7 tratamentos e 10 repetições e a função de ligação é a logística. Esse e os demais modelos utilizados nesse trabalho serão descritos nos itens (3.2.1), (3.2.2) e (3.2.3).

É interessante salientar que esse conjunto de dados foi analisado por Vieira (1998) usando o enfoque clássico para os modelos binomial, binomial truncado, ZIB e suas versões superdispersas. O autor verificou através do gráfico meio normal com envelope simulado que o melhor modelo foi o binomial truncado superdisperso com preditor linear dado por um polinômio de terceiro grau o que orientou até certo ponto o uso de polinômios com até quarto grau para a análise usando a metodologia Bayesiana, neste trabalho.

\subsubsection{Modelo binomial com superdispersão}

Uma escolha natural para a modelagem da proporção de ovos parasitados é o modelo de regressão binomial. Portanto, a função de probabilidade da variável $Y_{i}$, dados $m_{i}$ e $p_{i}$, é dada por

$$
f\left(y_{i} \mid m_{i}, p_{i}\right)=\left(\begin{array}{c}
m_{i} \\
y_{i}
\end{array}\right) p_{i}^{y_{i}}\left(1-p_{i}\right)^{m_{i}-y_{i}}, \quad 0 \leq p_{i} \leq 1
$$

em que $p_{i}$ é a probabilidade de um ovo ser parasitado, além disso, a esperança e a variância de $Y_{i}$ dado $m_{i}$ e $p_{i}$ são dadas, respectivamente, por

$$
E\left(Y_{i} \mid m_{i}, p_{i}\right)=m_{i} p_{i}
$$

e

$$
\operatorname{Var}\left(Y_{i} \mid m_{i}, p_{i}\right)=m_{i} p_{i}\left(1-p_{i}\right)
$$


O modelo linear generalizado permite modelar as proporções esperadas em termos de variáveis explanatórias $\boldsymbol{x}_{i}^{T}$ (efeitos de tratamentos e covariáveis) através de

$$
g\left(\mu_{i}\right)=\boldsymbol{x}_{i}^{T} \boldsymbol{\beta},
$$

sendo $\boldsymbol{\beta}$ um vetor de parâmetros a ser estimado, $\boldsymbol{x}_{i}^{T}$ a matriz de variáveis explanatórias e $g($.$) uma função de ligação adequada. No conjunto considerado de$ dados utiliza-se função de ligação logística, isto é,

$$
g\left(p_{i}\right)=\log \left(\frac{p_{i}}{1-p_{i}}\right)=\beta_{0}+\beta_{1} x_{i 1}+\beta_{2} x_{i 2}^{2}+\beta_{3} x_{i 3}^{3}+\beta_{4} x_{i 4}^{4},
$$

sendo $\beta_{j}$ o coeficiente do modelo de regressão, para $j=0,1,2,3,4, x_{i}=\left(z_{i}-\bar{z}\right)$ com $i=1,2, \ldots, 70, z_{i}=\log _{2}$ (número de fêmeas) e $\bar{z}=\frac{\log _{2} \text { (número de fêmeas) }}{\text { número de fêmeas }}$.

O modelo maximal, para os dados considerados, será aquele em que o número de fêmeas é usado como fator qualitativo, isto é, com sete níveis de tratamento.

Para a estimação de $\beta_{j}$ usando o método Bayesiano, assumem-se as seguintes distribuições a priori para os parâmetros do modelo de regressão:

$$
\beta_{j} \sim N\left(\mu_{j}, \sigma_{j}^{2}\right) ; \mu_{j}, \sigma_{j}^{2} \text { conhecidos }, \quad j=0,1, \ldots, q .
$$

em que $q$ é o número de parâmetros do modelo e considerando-se que os parâmetros $\beta_{j}$ são independentes, a função distribuição a priori conjunta para $\beta_{0}, \beta_{1}, \ldots, \beta_{q}$ pode ser dada por

$$
\begin{gathered}
\pi\left(\beta_{0}, \beta_{1}, \ldots, \beta_{q}\right) \propto \exp \left\{\frac{-1}{2 \sigma_{0}}\left(\beta_{0}-\mu_{0}\right)^{2}\right\} \exp \left\{\frac{-1}{2 \sigma_{1}}\left(\beta_{1}-\mu_{1}\right)^{2}\right\} \\
\ldots \exp \left\{\frac{-1}{2 \sigma_{q}}\left(\beta_{q}-\mu_{q}\right)^{2}\right\} .
\end{gathered}
$$

Além disso, a função de verossimilhança para o modelo binomial, dado em (14), é dada por

$$
L(\boldsymbol{p} ; \boldsymbol{y}, \boldsymbol{m})=\prod_{i=1}^{n}\left[\left(\begin{array}{c}
m_{i} \\
y_{i}
\end{array}\right) p_{i}^{y_{i}}\left(1-p_{i}\right)^{m_{i}-y_{i}}\right]
$$


$\operatorname{com} p_{i}=\frac{e^{\sum_{i=1}^{n} x_{i} \beta}}{1+e^{\sum_{i=1}^{n} x_{i} \beta}}$.

Logo,

$$
\begin{aligned}
L\left(\boldsymbol{\beta} ; \boldsymbol{y}, \boldsymbol{x}_{i}, \boldsymbol{m}\right) & \propto \prod_{i=1}^{n}\left[\left(\frac{e^{x_{i} \beta}}{1+e^{x_{i} \beta}}\right)^{y_{i}}\left(\frac{1}{1+e^{x_{i} \beta}}\right)^{m_{i}-y_{i}}\right] \\
& =\prod_{i=1}^{n}\left[\frac{e^{y_{i} x_{i} \beta}}{\left(1+e^{x_{i} \beta}\right)^{m_{i}}}\right]
\end{aligned}
$$

em que $\boldsymbol{x}_{i}=\left(x_{i 1}, x_{i 2}, \ldots, x_{i q}\right)^{T}, \boldsymbol{y}=\left(y_{1}, y_{2}, \ldots, y_{n}\right), \boldsymbol{m}=\left(m_{1}, m_{2}, \ldots, m_{n}\right)$ e $\boldsymbol{\beta}=\left(\beta_{0}, \beta_{1}, \ldots, \beta_{q}\right)$.

Multiplicando-se as expressões (17) e (18), encontra-se a distribuição a posteriori conjunta para $\beta_{0}, \beta_{1}, \ldots, \beta_{q}$, isto é,

$$
\begin{array}{r}
\pi\left(\boldsymbol{\beta} \mid \boldsymbol{x}_{i}, \boldsymbol{y}, \boldsymbol{m}\right) \propto \exp \left\{\frac{-1}{2 \sigma_{0}}\left(\beta_{0}-\mu_{0}\right)^{2}\right\} \exp \left\{\frac{-1}{2 \sigma_{1}}\left(\beta_{1}-\mu_{1}\right)^{2}\right\} \\
\ldots \exp \left\{\frac{-1}{2 \sigma_{q}}\left(\beta_{q}-\mu_{q}\right)^{2}\right\} \prod_{i=1}^{n}\left[\frac{e^{y_{i} x_{i} \beta}}{\left(1+e^{x_{i} \beta}\right)^{m_{i}}}\right] .
\end{array}
$$

Logo, a distribuição a posteriori condicional completa para $\beta_{j}$ com $j=0,1,2, \ldots, q$, necessária para o uso dos métodos MCMC, é dada por

$$
\pi\left(\beta_{j} \mid \boldsymbol{\beta}_{-j}, \boldsymbol{x}_{i}, \boldsymbol{y}, \boldsymbol{m}\right) \propto \exp \left\{\frac{-1}{2 \sigma_{j}}\left(\beta_{j}-\mu_{j}\right)^{2}\right\} \prod_{i=1}^{n}\left[\frac{e^{y_{i} x_{i} \beta}}{\left(1+e^{x_{i} \beta}\right)^{m_{i}}}\right],
$$

sendo $\boldsymbol{\beta}_{-\boldsymbol{j}}=\left(\beta_{0}, \ldots, \beta_{j-1}, \beta_{j+1}, \ldots, \beta_{q}\right)$.

Observando-se a distribuição a posteriori condicional completa de $\beta_{j}$, verifica-se que elas não apresentam formas de distribuições conhecidas. Portanto, utiliza-se o método MCMC usando o algoritmo Metropolis-Hastings para realizar as simulações dos parâmetros e obter as estimativas de $\beta_{0}, \beta_{1}, \ldots, \beta_{q}$.

Para fazer a análise dos dados, para cada modelo de regressão, gerase através da equação (16) uma cadeia com 100.000 valores para cada parâmetro, desprezando os primeiros 2.000 valores e selecionado 1 em cada 21 dos 98.000 valores restantes, formando uma amostra com 4.500 valores.

Entretanto, em muitas aplicações, ocorre variabilidade observada nos dados maior do que a esperada pelo modelo probabilístico, sendo essa característica 
bastante comum em estudos de Entomologia. A análise visual dos dados apresentados na seção (3.1), em que a variável resposta (y) é a proporção de ovos parasitados, indica que existe um excesso de zeros e uma variabilidade grande, provocados, provavelmente, pela variabilidade natural dos ovos dentro de cada grupo e pela variabilidade entre fêmeas.

Uma maneira simples de modelar a superdispersão é multiplicar a variância do modelo binomial por uma constante, isto é,

$$
\operatorname{Var}\left(Y_{i} \mid m_{i}, p_{i}\right)=\phi m_{i} p_{i}\left(1-p_{i}\right) .
$$

O fator de superdispersão, como é chamada a constante $\phi$, indica que a variabilidade extra-binomial ocasionada pelas observações $\boldsymbol{y}$ não depende do tamanho amostral $m_{i}$ e das probabilidades $p_{i}$. Se $\phi>1$, indica que a variabilidade dos dados é maior do que a variabilidade do modelo (superdispersão) enquanto que para $0<\phi<1$ tem-se que a variabilidade dos dados é menor do que a variabilidade do modelo (subdispersão), sendo a segunda situação mais difícil de ser encontrada na prática.

Para estimar a superdispersão usando a metodologia Bayesiana, assumem-se as distribuições a priori para $\beta_{j}$ dada pela equação (16) e para $\phi$ dada por

$$
\phi \sim \operatorname{Gama}(a, b)
$$

com $a$ e $b$ conhecidos. Nota-se ainda, que valores pequenos para $b$ resultam em uma distribuição a priori não informativa.

Com isso, a distribuição a posteriori conjunta para $\beta_{j}$ e $\phi$, incluindo o parâmetro de dispersão na função de verossimilhança dada em (18), é dada por

$$
\begin{gathered}
\pi\left(\boldsymbol{\beta}, \phi ; \boldsymbol{x}_{i}, \boldsymbol{y}, \boldsymbol{m}\right) \propto \phi^{a-1} \exp \{-b \phi\} \exp \left\{\frac{-1}{2 \sigma_{0}}\left(\beta_{0}-\mu_{0}\right)^{2}\right\} \exp \left\{\frac{-1}{2 \sigma_{1}}\left(\beta_{1}-\mu_{1}\right)^{2}\right\} \\
\ldots \exp \left\{\frac{-1}{2 \sigma_{q}}\left(\beta_{q}-\mu_{q}\right)^{2}\right\} \prod_{i=1}^{n}\left[\frac{e^{y_{i} x_{i} \beta}}{\left(1+e^{x_{i} \beta}\right)^{m_{i}}}\right]^{\phi} .
\end{gathered}
$$


Para obter a estimativa de $\phi$ é necessário usar as distribuições a posteriori condicionais completas para $\phi$ e $\beta_{j}$ dadas, respectivamente, por

$$
\pi\left(\phi \mid \boldsymbol{\beta}, \boldsymbol{x}_{i}, \boldsymbol{y}, \boldsymbol{m}\right) \propto \phi^{a-1} \exp \{-b \phi\} \prod_{i=1}^{n}\left[\frac{e^{y_{i} x_{i} \beta}}{\left(1+e^{x_{i} \beta}\right)^{m_{i}}}\right]^{\phi}
$$

e

$$
\pi\left(\beta_{j} \mid \boldsymbol{\beta}_{-j}, \phi, \boldsymbol{x}_{i}, \boldsymbol{y}, \boldsymbol{m}\right) \propto \exp \left\{\frac{-1}{2 \sigma_{j}}\left(\beta_{j}-\mu_{j}\right)^{2}\right\} \prod_{i=1}^{n}\left[\frac{e^{y_{i} x_{i} \beta}}{\left(1+e^{x_{i} \beta}\right)^{m_{i}}}\right]^{\phi} .
$$

Nota-se porém, que as distribuições a posteriori condicinais completas não apresentam formas de distribuições conhecidas, sendo necessário utilizar o método MCMC através do algoritmo Metropolis-Hastings para realizar as simulações dos parâmetros e obter a estimativa de $\phi$. Os valores de $\phi$ serão gerados através da equação (20) e será obtida uma amostra de 100.000 valores, desprezando os primeiros 2.000 valores e selecionando 1 em cada 21 dos 98.000 valores restantes.

Considerando-se, então, o modelo de regressão dado em (15) e fixandose o valor de $\hat{\hat{\phi}}$ nas distribuições a posteriori condicionais para que se obtenha a função de variância dada em (19), os valores de $\beta_{j}$ serão gerados por

$$
\pi\left(\beta_{j} \mid \boldsymbol{\beta}_{-j}, \boldsymbol{x}_{i}, \boldsymbol{y}, \boldsymbol{m}\right) \exp \left\{\frac{-1}{2 \sigma_{j}}\left(\beta_{j}-\mu_{j}\right)^{2}\right\} \prod_{i=1}^{n}\left[\frac{e^{y_{i} x_{i} \beta}}{\left(1+e^{x_{i} \beta}\right)^{m_{i}}}\right]^{\hat{\hat{\phi}}^{-1}}
$$

e através do DIC (Spiegelhalter et al., 1998) e do fator de Bayes (Kass \& Raftery, 1995) serão comparados os modelos. Além disso, serão apresentados gráficos dos valores preditos versus número de fêmeas para uma visualização dos diferentes modelos ajustados.

O critério de informação da deviance (DIC), para o caso particular do modelo binomial, definido por Spiegelhalter et al. (1998) é dado por:

$$
D I C=\bar{D}(\boldsymbol{p}, \phi ; \boldsymbol{y})+p_{D},
$$

sendo $\bar{D}(\boldsymbol{p}, \phi ; \boldsymbol{y})=E \boldsymbol{p} \mid \boldsymbol{y}(\boldsymbol{p}, \phi ; \boldsymbol{y})$ e $p_{D}$ obtidos através do desenvolvimento dado a 
seguir. O logaritmo da função de verossimilhança do modelo binomial é dado por

$$
\begin{aligned}
l(\boldsymbol{p}, \phi ; \boldsymbol{y}, \boldsymbol{m}) & =\log \left(\prod_{i=1}^{n} L\left(p_{i}, \phi, y_{i}, m_{i}\right)\right) \\
& \propto \frac{1}{\phi} \sum_{i=1}^{n}\left[y_{i} \log \left(p_{i}\right)+\left(m_{i}-y_{i}\right) \log \left(1-p_{i}\right)\right],
\end{aligned}
$$

e, portanto, a deviance residual fica

$$
D(\boldsymbol{p}, \phi ; \boldsymbol{y}) \propto \frac{-2}{\phi} \sum_{i=1}^{n}\left[y_{i} \log \left(p_{i}\right)+\left(m_{i}-y_{i}\right) \log \left(1-p_{i}\right)\right] .
$$

Logo,

$$
\begin{gathered}
\bar{D}(\boldsymbol{p}, \phi ; \boldsymbol{y})=E_{p \mid y}(D(\boldsymbol{p}, \phi ; \boldsymbol{y}))=E_{\boldsymbol{p} \mid \boldsymbol{y}}\left\{\frac{-2}{\phi} \sum_{i=1}^{n}\left[y_{i} \log \left(p_{i}\right)+\left(m_{i}-y_{i}\right) \log \left(1-p_{i}\right)\right]\right\} \\
D(\bar{p}, \phi ; \boldsymbol{y})=\frac{-2}{\phi} \sum_{i=1}^{n}\left[y_{i} \log \left(\bar{p}_{i}\right)\left(m_{i}-y_{i}\right) \log \left(1-\bar{p}_{i}\right)\right]
\end{gathered}
$$

e

$$
D I C=E_{p \mid y}\left\{\frac{-2}{\phi} \sum_{i=1}^{n}\left[y_{i} \log \left(p_{i}\right)+\left(m_{i}-y_{i}\right) \log \left(1-p_{i}\right)\right]\right\}+p_{D}
$$

em que $p_{i}=\frac{e^{x_{i} \beta}}{1+e^{x_{i} \beta}}$ e $p_{D}=\bar{D}(\boldsymbol{p}, \phi ; \boldsymbol{y})-D(\overline{\boldsymbol{p}}, \phi ; \boldsymbol{y})$.

O fator de Bayes será utilizado conjuntamente com o DIC. Para isso é necessário encontrar a função de verossimilhança marginal, utilizando métodos MCMC. No modelo binomial, essa função é dada por

$$
\hat{\pi}(\boldsymbol{y} \mid M)=\frac{1}{T} \sum_{t=1}^{T}\left\{\prod_{i=1}^{n}\left[\frac{e^{y_{i} x_{i} \beta^{(t)}}}{\left(1+e^{x_{i} \beta^{(t)}}\right)^{m_{i}}}\right]\right\},
$$

sendo $M$ o modelo corrente e $T$ o tamanho da amostra obtida usando o método MCMC.

Para determinar o fator de Bayes é necessário fazer a razão entre dois modelos, isto é,

$$
B=\frac{\hat{\pi}\left(\boldsymbol{y} \mid M_{1}\right)}{\hat{\pi}\left(\boldsymbol{y} \mid M_{2}\right)}
$$

Dessa forma, o fator de Bayes é um valor numérico baseado nos dados que determina o melhor modelo. Uma interpretação do fator de Bayes sugerido por Kass \& Raftery (1995), é dada na Tabela 1. 
Tabela 1. Interpretação do fator de Bayes

\begin{tabular}{ccc}
\hline$B$ & $2 \log (B)$ & Evidência a favor de $M_{1}$ \\
\hline$<1$ & $<0$ & Negativa (apoia $\left.M_{2}\right)$ \\
$1 \vdash 3$ & $0 \vdash 2$ & Fraca \\
$3 \vdash 20$ & $2 \vdash 6$ & Positiva \\
$20 \vdash 150$ & $6 \vdash 10$ & Forte \\
$>50$ & $>10$ & Muito Forte
\end{tabular}

\subsubsection{Modelo binomial truncado superdisperso}

Considerando que o pesquisador não tem interesse no número de ovos que não foram parasitados, pode-se utilizar um modelo probabilístico que exclua os valores nulos, isto é, o modelo binomial truncado com $y_{i}>0$. A função de probabilidade da variável $Y_{i}$ é dada por

$$
f\left(y_{i} \mid m_{i}, p_{i}\right)=\frac{\left(\begin{array}{c}
m_{i} \\
y_{i}
\end{array}\right) p_{i}^{y_{i}}\left(1-p_{i}\right)^{m_{i}-y_{i}}}{1-\left(1-p_{i}\right)^{m_{i}}} \quad 0<p_{i} \leq 1
$$

sendo que

$$
E\left(Y_{i} \mid p_{i}, m_{i}\right)=\frac{m_{i} p_{i}}{1-\left(1-p_{i}\right)^{m_{i}}}=\mu_{i}
$$

$\mathrm{e}$

$$
\operatorname{Var}\left(Y_{i} \mid p_{i}, m_{i}\right)=\mu_{i}\left[1-\mu_{i}+p_{i}\left(m_{i}-1\right)\right] .
$$

O modelo binomial truncado permite modelar dados ignorando o excesso de zeros existentes mas, eventualmente, superdispersão devida a outras causas (probabilidade desigual de sucesso ou dados agrupados) podem estar presentes. Um modelo simples que pode ser eficiente para modelar fontes de dispersão é um modelo binomial truncado superdisperso com um fator de heterocedasticidade, isto é, com

$$
\operatorname{Var}\left(Y_{i} \mid p_{i}, m_{i}, \phi\right)=\mu_{i}\left[1-\mu_{i}+p_{i}\left(m_{i}-1\right)\right] \phi
$$

A modelagem das proporções esperadas $p_{i}$ será feita de forma semelhante ao proposto para o modelo binomial, isto é, com ligação logística e preditores lineares com polinômios até de quarto grau, de acordo com (15). 
Tem-se, então que

$$
L(\boldsymbol{p} ; \boldsymbol{y}, \boldsymbol{m})=\prod_{i=1}^{n}\left[\frac{\left(\begin{array}{c}
m_{i} \\
y_{i}
\end{array}\right) p_{i}^{y_{i}}\left(1-p_{i}\right)^{m_{i}-y_{i}}}{1-\left(1-p_{i}\right)^{m_{i}}}\right]
$$

$\operatorname{com} p_{i}=\frac{e^{\sum_{i=1}^{n} x_{i} \beta}}{1+e^{\sum_{i=1}^{n} x_{i} \beta}}$

Logo,

$$
\begin{aligned}
L\left(\boldsymbol{\beta} ; \boldsymbol{x}_{i}, \boldsymbol{y}, \boldsymbol{m}\right) & \propto \prod_{i=1}^{n}\left[\frac{\left(\frac{e^{x_{i} \beta}}{1+e^{x_{i} \beta}}\right)^{y_{i}}\left(\frac{1}{1+e^{x_{i} \beta}}\right)^{m_{i}-y_{i}}}{1-\left(\frac{1}{1+e^{x_{i} \beta}}\right)^{m_{i}}}\right] \\
& =\prod_{i=1}^{n}\left[\frac{\frac{e^{y_{i} x_{i} \beta}}{\left(1+e^{x_{i} \beta}\right)^{m_{i}}}}{\frac{\left(1+e^{x_{i} \beta}\right)^{m_{i}}-1}{\left(1+e^{x_{i} \beta}\right)^{m_{i}}}}\right]=\prod_{i=1}^{n} \frac{e^{y_{i} x_{i} \beta}}{\left(1+e^{x_{i} \beta}\right)^{m_{i}}-1} .
\end{aligned}
$$

Para a estimação dos parâmetros do modelo pelo método Bayesiano, as distribuições a priori escolhidas para $\beta_{0}, \beta_{1}, \ldots, \beta_{q}$ são dadas da mesma forma utilizada para o modelo binomial, conforme expressão (16). Portanto, considerando independência entre os parâmetros $\beta_{0}, \beta_{1}, \ldots, \beta_{q}$, a função distribuição a priori conjunta é dada pela expressão (17).

Para encontrar a distribuição a posteriori conjunta para $\beta_{0}, \beta_{1}, \ldots, \beta_{q}$, multiplicam-se as expressões (17) e (23), obtendo-se assim

$$
\begin{aligned}
\pi\left(\boldsymbol{\beta} \mid \boldsymbol{x}_{i}, \boldsymbol{y}, \boldsymbol{m}\right) \propto & \exp \left\{\frac{-1}{2 \sigma_{0}}\left(\beta_{0}-\mu_{0}\right)^{2}\right\} \exp \left\{\frac{-1}{2 \sigma_{1}}\left(\beta_{1}-\mu_{1}\right)^{2}\right\} \\
\ldots & \exp \left\{\frac{-1}{2 \sigma_{q}}\left(\beta_{q}-\mu_{q}\right)^{2}\right\} \prod_{i=1}^{n}\left[\frac{e^{y_{i} x_{i} \beta}}{\left(1+e^{x_{i} \beta}\right)^{m_{i}}-1}\right] .
\end{aligned}
$$

Logo a distribuição a posteriori condicional completa para $\beta_{j}$, em que $j=0,1, \ldots, q$, é dada por

$$
\pi\left(\beta_{j} \mid \boldsymbol{\beta}_{-j}, \boldsymbol{x}_{i}, \boldsymbol{y}, \boldsymbol{m}\right) \propto \exp \left\{\frac{-1}{2 \sigma_{j}}\left(\beta_{j}-\mu_{j}\right)^{2}\right\} \prod_{i=1}^{n}\left[\frac{e^{y_{i} x_{i} \beta}}{\left(1+e^{x_{i} \beta}\right)^{m_{i}}-1}\right] .
$$

A exemplo do modelo binomial, nota-se que a distribuição a posteriori condicional completa para $\beta_{j}$ não têm uma forma conhecida. Portanto, utiliza-se o método MCMC usando o algoritmo Metropolis-Hastings para realizar as simulações dos parâmetros e obter as estimativas $\beta_{0}, \beta_{1}, \ldots, \beta_{q}$. 
Para a análise dos dados, usando o modelo binomial truncado, gerase uma cadeia com 100.000 valores para cada parâmetro, desprezando os primeiros 2.000 valores e selecionado 1 em cada 21 dos 98.000 valores restantes, formando uma amostra com 4.500 valores, sendo esses gerados através da distribuição a priori dada pela equação (16).

Para a estimação do parâmetro de dispersão, utilizam-se as distribuições a priori para $\phi$ dada em (20) e para $\boldsymbol{\beta}$ dada em (16) e a função de verossimilhança dada em (23), incluindo o parâmetro de dispersão. Com isso, a distribuição a posteriori conjunta é dada por

$$
\begin{array}{r}
\pi\left(\boldsymbol{\beta}, \phi ; \boldsymbol{x}_{i}, \boldsymbol{y}, \boldsymbol{m}\right) \propto \phi^{a-1} \exp \{-b \phi\} \exp \left\{\frac{-1}{2 \sigma_{0}}\left(\beta_{0}-\mu_{0}\right)^{2}\right\} \exp \left\{\frac{-1}{2 \sigma_{1}}\left(\beta_{1}-\mu_{1}\right)^{2}\right\} \\
\ldots \exp \left\{\frac{-1}{2 \sigma_{q}}\left(\beta_{q}-\mu_{q}\right)^{2}\right\} \prod_{i=1}^{n}\left[\frac{e^{y_{i} x_{i} \beta}}{\left(1+e^{x_{i} \beta}\right)^{m_{i}}-1}\right]^{\phi} .
\end{array}
$$

Para obter a estimativa de $\phi$ é necessário usar as distribuições a posteriori condicionais para $\phi$ e $\beta_{j}$ dadas, respectivamente, por

$$
\pi\left(\phi \mid \boldsymbol{\beta}, \boldsymbol{x}_{i}, \boldsymbol{y}, \boldsymbol{m}\right) \propto \phi^{a-1} \exp \{-b \phi\} \prod_{i=1}^{n}\left[\frac{e^{y_{i} x_{i} \beta}}{\left(1+e^{x_{i} \beta}\right)^{m_{i}}-1}\right]^{\phi}
$$

e

$$
\pi\left(\beta_{j} \mid \boldsymbol{\beta}_{-j}, \phi, \boldsymbol{x}_{i}, \boldsymbol{y}, \boldsymbol{m}\right) \propto \exp \left\{\frac{-1}{2 \sigma_{j}}\left(\beta_{j}-\mu_{j}\right)^{2}\right\} \prod_{i=1}^{n}\left[\frac{e^{y_{i} x_{i} \beta}}{\left(1+e^{x_{i} \beta}\right)^{m_{i}}-1}\right]^{\phi} .
$$

Nota-se que as distribuições a posteriori condicionais completas não apresentam formas de distribuições conhecidas, sendo necessário utilizar métodos MCMC através do algoritmo Metropolis-Hastings para realizar as simulações de $\phi$.

Considerando-se, então, o modelo de regressão dado em (15) e fixandose o valor de $\hat{\hat{\phi}}$ nas distribuições a posteriori condicionais para que se obtenha a função de variância dada em (22), os valores de $\beta_{j}$ serão gerados por

$$
\pi\left(\beta_{j}, \mid \boldsymbol{\beta}_{-j}, \boldsymbol{x}_{i}, \boldsymbol{y}, \boldsymbol{m}\right) \propto \exp \left\{\frac{-1}{2 \sigma_{j}}\left(\beta_{j}-\mu_{j}\right)^{2}\right\} \prod_{i=1}^{n}\left[\frac{e^{y_{i} x_{i} \beta}}{\left(1+e^{x_{i} \beta}\right)^{m_{i}}-1}\right]^{\hat{\hat{\phi}}^{-1}} .
$$

A expressão do DIC usada para escolher o polinômio do modelo de regressão que melhor se ajusta aos dados é dada por

$$
D I C=\bar{D}(\boldsymbol{p}, \phi ; \boldsymbol{y})+p_{D},
$$


sendo que o logaritmo da função de verossimilhança desse modelo é dado por

$$
\begin{aligned}
l(\boldsymbol{p}, \phi ; \boldsymbol{y}, \boldsymbol{m}) & =\log \left(\prod_{i=1}^{n} L\left(p_{i}, \phi ; y_{i}, m_{i}\right)\right) \\
& \propto \frac{1}{\phi} \sum_{i=1}^{n}\left\{y_{i} \log \left(p_{i}\right)+\left(m_{i}-y_{i}\right) \log \left(1-p_{i}\right)-\log \left[1-\left(1-p_{i}\right)^{m_{i}}\right]\right\},
\end{aligned}
$$

e, portanto,

$$
D(\boldsymbol{p}, \phi ; \boldsymbol{y}) \propto \frac{-2}{\phi} \sum_{i=1}^{n}\left\{y_{i} \log \left(p_{i}\right)+\left(m_{i}-y_{i}\right) \log \left(1-p_{i}\right)-\log \left[1-\left(1-p_{i}\right)^{m_{i}}\right]\right\} .
$$

Logo,

$$
\begin{aligned}
& \bar{D}(\boldsymbol{p}, \phi ; \boldsymbol{y})=E_{p \mid y}(D(\boldsymbol{p}, \phi ; \boldsymbol{y}))= E_{p \mid y}\left\{\frac { - 2 } { \phi } \sum _ { i = 1 } ^ { n } \left[y_{i} \log \left(p_{i}\right)+\left(m_{i}-y_{i}\right) \log \left(1-p_{i}\right)\right.\right. \\
&\left.\left.-\log \left[1-\left(1-p_{i}\right)^{m_{i}}\right]\right]\right\}, \\
& D(\overline{\boldsymbol{p}}, \phi ; y)=\frac{-2}{\phi} \sum_{i=1}^{n}\left\{y_{i} \log \left(p_{i}\right)+\left(m_{i}-y_{i}\right) \log \left(1-p_{i}\right)-\log \left[1-\left(1-p_{i}\right)^{m_{i}}\right]\right\}
\end{aligned}
$$

$\mathrm{e}$

$$
D I C=E_{p \mid y}\left\{\frac{-2}{\phi} \sum_{i=1}^{n}\left[y_{i} \log \left(p_{i}\right)+\left(m_{i}-y_{i}\right) \log \left(1-p_{i}\right)-\log \left[1-\left(1-p_{i}\right)^{m_{i}}\right]\right]\right\}+p_{D}
$$

em que $p_{i}=\frac{e^{x_{i} \beta}}{1+e^{x_{i} \beta}}$ e $p_{D}=\bar{D}(\boldsymbol{p}, \phi ; \boldsymbol{y})-D(\overline{\boldsymbol{p}}, \phi ; \boldsymbol{y})$.

A função de verossimilhança marginal, necessária para estimar o fator de Bayes, no modelo binomial truncado é dada por

$$
\hat{\pi}(\boldsymbol{y} \mid M)=\frac{1}{T} \sum_{t=1}^{T}\left\{\prod_{i=1}^{n}\left[\frac{e^{y_{i} x_{i} \beta^{(t)}}}{\left(1+e^{x_{i} \beta^{(t)}}\right)^{m_{i}}-1}\right]\right\},
$$

sendo $M$ o modelo corrente e $T$ o tamanho da amostra obtida usando o método MCMC.

\subsubsection{Modelo binomial inflacionado de zeros superdisperso}

Um caso particular de distribuição de mistura Bernoulli-binomial são os modelos inflacionados de zeros. Segundo Ghosh et al. (2002), a variável aleatória 
$Y_{i}$ pode ser representada por $Y_{i}=V_{i}\left(1-B_{i}\right)$, sendo $B_{i}$ uma variável aleatória Bernoulli, isto é,

$$
B_{i} \sim B\left(w_{i}\right)
$$

sendo $w_{i}$ a probabilidade de uma fêmea parasitar e $V_{i}$ uma variável aleatória com distribuição binomial, isto é, $V_{i} \sim \operatorname{Bin}\left(m_{i}, p_{i}\right)$.

Tem-se, então, que $Y_{i}$ tem uma distribuição binomial inflacionada de zeros (ZIB), com função de probabilidades dada por

$$
f\left(y_{i} \mid m_{i}, p_{i}, w_{i}\right)= \begin{cases}w_{i}+\left(1-w_{i}\right)\left(1-p_{i}\right)^{m_{i}}, & y_{i}=0 \\
\left(1-w_{i}\right)\left(\begin{array}{c}
m_{i} \\
y_{i}
\end{array}\right) p_{i}^{y_{i}}\left(1-p_{i}\right)^{m_{i}-y_{i}}, & y_{i}=1,2, \cdots, m_{i},\end{cases}
$$

sendo que $0 \leq w_{i}<1$ e $0 \leq p_{i}<1$. Para o caso particular em que $w_{i}=0$, o modelo reduz-se à distribuição binomial $\left(m_{i}, p_{i}\right)$.

Considerando $B_{i}$ uma variável latente, pois não é possível saber se a fêmea não parasitou por sua incapacidade de parasitar devido ao resultado aleatório de não ter ocorrido o contato com o ovo, tem-se que $b_{i}=1$ se $y_{i}=0$ e $b_{i}=0$ se $y_{i}>0$. Então, a função de probabilidade de $Y_{i}$, também pode ser escrita como

$$
f\left(y_{i} \mid m_{i}, p_{i}, w_{i}, b_{i}\right) \propto b_{i}\left[w_{i}+\left(1-w_{i}\right)\left(1-p_{i}\right)^{m_{i}}\right]+\left(1-b_{i}\right)\left[\left(1-w_{i}\right) p_{i}^{y_{i}}\left(1-p_{i}\right)^{m_{i}-y_{i}}\right]
$$

e, portanto,

$$
E\left(Y_{i} \mid m_{i}, p_{i}, w_{i}\right)=\left(1-w_{i}\right) m_{i} p_{i}=\mu_{i}
$$

e

$$
\operatorname{Var}\left(Y_{i} \mid m_{i}, p_{i}, w_{i}\right)=\mu_{i}\left[1-p_{i}+\left(\frac{w_{i}}{1-w_{i}}\right) \mu_{i}\right] .
$$

$\mathrm{Na}$ versão superdispersa desse modelo, com fator de heterogeneidade constante, assume-se que

$$
\operatorname{Var}\left(Y_{i} \mid m_{i}, p_{i}, w_{i}, \phi\right)=\mu_{i}\left[1-p_{i}+\left(\frac{w_{i}}{1-w_{i}}\right) \mu_{i}\right] \phi
$$

O modelo ZIB tem dois vetores de parâmetros a serem estimados, sendo então, necessárias, para o ajuste do modelo, duas funções de ligação. A função de 
ligação das proporções positivas, $\boldsymbol{p}$, é dada em (15). Para as proporções de zeros, $\boldsymbol{w}$, o modelo de regressão proposto é dado por

$$
g\left(w_{i}\right)=\log \left(\frac{w_{i}}{1-w_{i}}\right)=\gamma_{0}+\gamma_{1} g_{i}+\gamma_{2} g_{i}^{2}+\gamma_{3} g_{i}^{3}+\gamma_{4} g_{i}^{4},
$$

sendo $\gamma_{k}$ o coeficiente do modelo de regressão para $k=0,1,2,3,4, g_{i}=\left(z_{i}-\bar{z}\right)$, $i=1,2, \ldots, 70, z_{i}=\log _{2}$ (número de fêmeas) e $\bar{z}=\frac{\log _{2} \text { (número de fêmeas) }}{\text { número de fêmeas }}$.

Para se obterem as estimativas de $\boldsymbol{\beta}$ e $\boldsymbol{\gamma}$ usando a metodologia Bayesiana, consideram-se que $\boldsymbol{\beta}$ e $\boldsymbol{\gamma}$ são variáveis aleatórias, com distribuições a priori, dadas por

(i) $\beta_{j} \sim N\left(\mu_{j}, \sigma_{j}^{2}\right) ; \mu_{j}, \sigma_{j}^{2}$ conhecidos $, j=1, \ldots, q_{1}$

(ii) $\gamma_{k} \sim N\left(\eta_{k}, \nu_{k}^{2}\right) ; \eta_{k}, \nu_{k}^{2}$ conhecidos $, k=1, \ldots, q_{2}$.

Além disso, assumindo independência entre as distribuições a priori para os parâmetros, a função distribuição a priori conjunta para $\beta_{0}, \beta_{1}, \ldots, \beta_{q_{1}}$ e $\gamma_{0}, \gamma_{1}, \ldots, \gamma_{q_{2}}$ é dada por

$$
\begin{aligned}
\pi(\boldsymbol{\beta}, \boldsymbol{\gamma}) \propto & \exp \left\{\frac{-1}{2 \sigma_{0}}\left(\beta_{0}-\mu_{0}\right)^{2}\right\} \exp \left\{\frac{-1}{2 \sigma_{1}}\left(\beta_{1}-\mu_{1}\right)^{2}\right\} \ldots \\
& \exp \left\{\frac{-1}{2 \sigma_{q_{1}}}\left(\beta_{q_{1}}-\mu_{q_{1}}\right)^{2}\right\} \exp \left\{\frac{-1}{2 \nu_{0}}\left(\gamma_{0}-\eta_{0}\right)^{2}\right\} \\
& \exp \left\{\frac{-1}{2 \nu_{1}}\left(\gamma_{1}-\eta_{1}\right)^{2}\right\} \ldots \exp \left\{\frac{-1}{2 \nu_{q_{2}}}\left(\gamma_{q_{2}}-\eta_{q_{2}}\right)^{2}\right\} .
\end{aligned}
$$

A função de verossimilhança, para o modelo ZIB com densidade dada em (25), em que $p_{i}=\frac{e^{x_{i} \beta}}{1+e^{x_{i} \beta}}$ e $w_{i}=\frac{e^{g_{i} \gamma}}{1+e^{g_{i} \gamma}}$ é representada por

$$
\begin{aligned}
L\left(\boldsymbol{\beta}, \boldsymbol{\gamma}, \boldsymbol{x}_{i}, \boldsymbol{g}_{i}, \boldsymbol{y}, \boldsymbol{m}, \boldsymbol{b}\right) & \propto \prod_{i=1}^{n} b_{i}\left[\frac{e^{g_{i} \gamma}}{\left(1+e^{g_{i} \gamma}\right)}+\left(\frac{1}{1+e^{g_{i} \gamma}}\right)\left(\frac{1}{1+e^{x_{i} \beta}}\right)^{m_{i}}\right]+ \\
& +\prod_{i=1}^{n}\left(1-b_{i}\right)\left[\frac{1}{\left(1+e^{g_{i} \gamma}\right)}\left(\frac{e^{x_{i} \beta}}{1+e^{x_{i} \beta}}\right)^{y_{i}}\left(\frac{1}{1+e^{x_{i} \beta}}\right)^{m_{i}-y_{i}}\right] \\
& =\prod_{i=1}^{n} b_{i}\left[\frac{1}{1+e^{g_{i} \gamma}}\left(e^{g_{i} \gamma}+\frac{1}{\left(1+e^{x_{i} \beta}\right)^{m_{i}}}\right)\right]+ \\
& +\prod_{i=1}^{n}\left(1-b_{i}\right)\left[\left(\frac{1}{1+e^{g_{i} \gamma}}\right) \frac{e^{y_{i} x_{i} \beta}}{\left(1+e^{x_{i} \beta}\right)^{m_{i}}}\right]
\end{aligned}
$$


sendo $\boldsymbol{x}_{i}=\left(x_{i 1}, x_{i 2}, \ldots, x_{i q_{1}}\right)^{T}$ e $\boldsymbol{g}_{i}=\left(g_{i 1}, g_{i 2}, \ldots, g_{i q_{2}}\right)^{T}$.

Multiplicando-se as expressões (29) e (30) obtém-se a distribuição a posteriori conjunta para $\beta_{0}, \beta_{1}, \ldots, \beta_{q_{1}}$ e $\gamma_{0}, \gamma_{1}, \ldots, \gamma_{q_{2}}$, dada por

$$
\begin{aligned}
\pi\left(\boldsymbol{\beta}, \boldsymbol{\gamma} \mid \boldsymbol{x}_{i}, \boldsymbol{g}_{i}, \boldsymbol{y}, \boldsymbol{m}, \boldsymbol{b}\right) \propto & \exp \left\{\frac{-1}{2 \sigma_{0}}\left(\beta_{0}-\mu_{0}\right)^{2}\right\} \exp \left\{\frac{-1}{2 \sigma_{1}}\left(\beta_{1}-\mu_{1}\right)^{2}\right\} \\
& \ldots \exp \left\{\frac{-1}{2 \sigma_{q_{1}}}\left(\beta_{q_{1}}-\mu_{q_{1}}\right)^{2}\right\} \exp \left\{\frac{-1}{2 \nu_{0}}\left(\gamma_{0}-\eta_{0}\right)^{2}\right\} \\
& \exp \left\{\frac{-1}{2 \nu_{1}}\left(\gamma_{1}-\eta_{1}\right)^{2}\right\} \ldots \exp \left\{\frac{-1}{2 \nu_{q_{2}}}\left(\gamma_{q_{2}}-\eta_{q_{2}}\right)^{2}\right\} \\
& \left\{\prod_{i=1}^{n} b_{i}\left[\frac{1}{1+e^{g_{i} \gamma}}\left(e^{g_{i} \gamma}+\frac{1}{\left(1+e^{x_{i} \beta}\right)^{m_{i}}}\right)\right]+\right. \\
& \left.+\prod_{i=1}^{n}\left(1-b_{i}\right)\left[\left(\frac{1}{1+e^{g_{i} \gamma}}\right) \frac{e^{y_{i} x_{i} \beta}}{\left(1+e^{x_{i} \beta}\right)^{m_{i}}}\right]\right\} .
\end{aligned}
$$

Logo, as distribuições a posteriori condicionais completas para $\beta_{j}$ e $\gamma_{k}$, com $j=1,2, \ldots, q_{1}$ e $k=1,2, \ldots, q_{2}$, necessárias para o uso dos métodos MCMC, são dadas por

$$
\begin{aligned}
\pi\left(\beta_{j} \mid \boldsymbol{\beta}_{-j}, \boldsymbol{\gamma}, \boldsymbol{x}_{i}, \boldsymbol{g}_{i}, \boldsymbol{y}, \boldsymbol{m}, \boldsymbol{b}\right) \propto & \exp \left\{\frac{-1}{2 \sigma_{j}}\left(\beta_{j}-\mu_{j}\right)^{2}\right\} \\
& \left\{\prod_{i=1}^{n} b_{i}\left[\frac{1}{1+e^{g_{i} \gamma}}\left(e^{g_{i} \gamma}+\frac{1}{\left(1+e^{x_{i} \beta}\right)^{m_{i}}}\right)\right]+\right. \\
+ & \left.+\prod_{i=1}^{n}\left(1-b_{i}\right)\left[\left(\frac{1}{1+e^{g_{i} \gamma}}\right) \frac{e^{y_{i} x_{i} \beta}}{\left(1+e^{x_{i} \beta}\right)^{m_{i}}}\right]\right\},
\end{aligned}
$$

e

$$
\begin{aligned}
\pi\left(\gamma_{k} \mid \boldsymbol{\gamma}_{-k}, \boldsymbol{\beta}, \boldsymbol{x}_{i}, \boldsymbol{g}_{i}, \boldsymbol{y}, \boldsymbol{m}, \boldsymbol{b}\right) \propto & \exp \left\{\frac{-1}{2 \nu_{k}}\left(\gamma_{k}-\eta_{k}\right)^{2}\right\} \\
& \left\{\prod_{i=1}^{n} b_{i}\left[\frac{1}{1+e^{g_{i} \gamma}}\left(e^{g_{i} \gamma}+\frac{1}{\left(1+e^{x_{i} \beta}\right)^{m_{i}}}\right)\right]+\right. \\
+ & \left.\prod_{i=1}^{n}\left(1-b_{i}\right)\left[\left(\frac{1}{1+e^{g_{i} \gamma}}\right) \frac{e^{y_{i} x_{i} \beta}}{\left(1+e^{x_{i} \beta}\right)^{m_{i}}}\right]\right\} .
\end{aligned}
$$

Nota-se, através das distribuições a posteriori condicionais, que os parâmetros $\beta_{j}$ e $\gamma_{k}$ não apresentam formas de distribuições conhecidas. Portanto, 
utiliza-se o método MCMC usando o algoritmo Metropolis-Hastings para realizar as simulações dos parâmetros e obter as estimativas de $\boldsymbol{\beta}$ e $\boldsymbol{\gamma}$.

Para fazer a análise dos dados usando o modelo ZIB, será gerada uma cadeia com 100.000 valores para cada parâmetro, desprezando os primeiros 2.000 valores e selecionando um a cada 21 dos 98.000 valores restantes, formando uma amostra de 4.500 valores, os quais foram gerados usando as distribuições a priori dadas pelas equações (i) e (ii) em (28).

Para a estimação do parâmetro de dispersão, utilizam-se as distribuições a priori para $\phi$ dada em (20), para $\boldsymbol{\beta}$ e $\boldsymbol{\gamma}$ dadas em (28) e a função de verossimilhança dada em (30), incluindo o parâmetro de dispersão. Com isso, a distribuição a posteriori conjunta é dada por

$$
\begin{aligned}
\pi\left(\boldsymbol{\beta}, \phi ; \boldsymbol{x}_{i}, \boldsymbol{g}_{i}, \boldsymbol{y}, \boldsymbol{m}\right) \propto & \phi^{a-1} \exp \{-b \phi\} \exp \left\{\frac{-1}{2 \sigma_{0}}\left(\beta_{0}-\mu_{0}\right)^{2}\right\} \exp \left\{\frac{-1}{2 \sigma_{1}}\left(\beta_{1}-\mu_{1}\right)^{2}\right\} \\
& \ldots \exp \left\{\frac{-1}{2 \sigma_{q_{1}}}\left(\beta_{q_{1}}-\mu_{q_{1}}\right)^{2}\right\} \exp \left\{\frac{-1}{2 \nu_{0}}\left(\gamma_{0}-\eta_{0}\right)^{2}\right\} \\
& \exp \left\{\frac{-1}{2 \nu_{1}}\left(\gamma_{1}-\eta_{1}\right)^{2}\right\} \ldots \exp \left\{\frac{-1}{2 \nu_{q_{2}}}\left(\gamma_{q_{2}}-\eta_{q_{2}}\right)^{2}\right\} \\
& \left\{\prod_{i=1}^{n} b_{i}\left[\frac{1}{1+e^{g_{i} \gamma}}\left(e^{g_{i} \gamma}+\frac{1}{\left(1+e^{x_{i} \beta}\right)^{m_{i}}}\right)\right]+\right. \\
& \left.+\prod_{i=1}^{n}\left(1-b_{i}\right)\left[\left(\frac{1}{1+e^{g_{i} \gamma}}\right) \frac{e^{y_{i} x_{i} \beta}}{\left(1+e^{x_{i} \beta}\right)^{m_{i}}}\right]^{\phi}\right\} .
\end{aligned}
$$

Para obter a estimativa de $\phi$ é necessário usar as distribuições a posteriori condicionais completas para $\phi, \boldsymbol{\beta}$ e $\boldsymbol{\gamma}$ dadas, respectivamente, por

$$
\begin{aligned}
\pi\left(\phi \mid \boldsymbol{\beta}, \boldsymbol{\gamma}, \boldsymbol{x}_{i}, \boldsymbol{g}_{i}, \boldsymbol{y}, \boldsymbol{m}\right) \propto & \phi^{a-1} \exp \{-b \phi\}\left\{\prod_{i=1}^{n} b_{i}\left[\frac{1}{1+e^{g_{i} \gamma}}\left(e^{g_{i} \gamma}+\frac{1}{\left(1+e^{x_{i} \beta}\right)^{m_{i}}}\right)\right]+\right. \\
& \left.+\prod_{i=1}^{n}\left(1-b_{i}\right)\left[\left(\frac{1}{1+e^{g_{i} \gamma}}\right) \frac{e^{y_{i} x_{i} \beta}}{\left(1+e^{x_{i} \beta}\right)^{m_{i}}}\right]^{\phi}\right\}
\end{aligned}
$$




$$
\begin{aligned}
\pi\left(\beta_{j} \mid \boldsymbol{\beta}_{-j}, \phi, \gamma, \boldsymbol{x}_{i}, \boldsymbol{g}_{i}, \boldsymbol{y}, \boldsymbol{m}\right) \propto & \exp \left\{\frac{-1}{2 \sigma_{j}}\left(\beta_{j}-\mu_{j}\right)^{2}\right\} \\
& \left\{\prod_{i=1}^{n} b_{i}\left[\frac{1}{1+e^{g_{i} \gamma}}\left(e^{g_{i} \gamma}+\frac{1}{\left(1+e^{x_{i} \beta}\right)^{m_{i}}}\right)\right]+\right. \\
& \left.+\prod_{i=1}^{n}\left(1-b_{i}\right)\left[\left(\frac{1}{1+e^{g_{i} \gamma}}\right) \frac{e^{y_{i} x_{i} \beta}}{\left(1+e^{x_{i} \beta}\right)^{m_{i}}}\right]^{\phi}\right\}
\end{aligned}
$$

$\mathrm{e}$

$$
\begin{aligned}
\pi\left(\gamma_{k} \mid \gamma_{-k}, \phi, \boldsymbol{\beta}, \boldsymbol{x}_{i}, \boldsymbol{g}_{i}, \boldsymbol{y}, \boldsymbol{m}\right) \propto & \exp \left\{\frac{-1}{2 \nu_{k}}\left(\gamma_{k}-\eta_{k}\right)^{2}\right\} \\
& \left\{\prod_{i=1}^{n} b_{i}\left[\frac{1}{1+e^{g_{i} \gamma}}\left(e^{g_{i} \gamma}+\frac{1}{\left(1+e^{x_{i} \beta}\right)^{m_{i}}}\right)\right]+\right. \\
& \left.+\prod_{i=1}^{n}\left(1-b_{i}\right)\left[\left(\frac{1}{1+e^{g_{i} \gamma}}\right) \frac{e^{y_{i} x_{i} \beta}}{\left(1+e^{x_{i} \beta}\right)^{m_{i}}}\right]^{\phi}\right\} .
\end{aligned}
$$

Nota-se que as distribuições a posteriori condicionais completas não apresentam formas de distribuições conhecidas, sendo necessário utilizar métodos MCMC através do algoritmo Metropolis-Hastings para realizar as simulações e obter a estimativa de $\phi$.

Considerando-se, então, os modelos de regressões dados em (15) e (27) e fixando-se o valor de $\hat{\hat{\phi}}$ nas distribuições a posteriori condicionais para que se obtenha a função de variância dada em (26), os valores de $\beta_{j}$ serão gerados por

$$
\begin{aligned}
\pi\left(\beta_{j} \mid \boldsymbol{\beta}_{-j}, \boldsymbol{\gamma}, \boldsymbol{x}_{i}, \boldsymbol{g}_{i}, \boldsymbol{y}, \boldsymbol{m}, \boldsymbol{b}\right) \propto & \exp \left\{\frac{-1}{2 \sigma_{j}}\left(\beta_{j}-\mu_{j}\right)^{2}\right\} \\
& \left\{\prod_{i=1}^{n} b_{i}\left[\frac{1}{1+e^{g_{i} \gamma}}\left(e^{g_{i} \gamma}+\frac{1}{\left(1+e^{x_{i} \beta}\right)^{m_{i}}}\right)\right]+\right. \\
& \left.+\prod_{i=1}^{n}\left(1-b_{i}\right)\left[\left(\frac{1}{1+e^{g_{i} \gamma}}\right) \frac{e^{y_{i} x_{i} \beta}}{\left(1+e^{x_{i} \beta}\right)^{m_{i}}}\right]^{\hat{\phi}^{-1}}\right\},
\end{aligned}
$$


e

$$
\begin{aligned}
\pi\left(\gamma_{k} \mid \boldsymbol{\gamma}_{-k}, \boldsymbol{\beta}, \boldsymbol{x}_{i}, \boldsymbol{g}_{i}, \boldsymbol{y}, \boldsymbol{m}, \boldsymbol{b}\right) \propto & \exp \left\{\frac{-1}{2 \nu_{k}}\left(\gamma_{k}-\eta_{k}\right)^{2}\right\} \\
& \left\{\prod_{i=1}^{n} b_{i}\left[\frac{1}{1+e^{g_{i} \gamma}}\left(e^{g_{i} \gamma}+\frac{1}{\left(1+e^{x_{i} \beta}\right)^{m_{i}}}\right)\right]+\right. \\
& \left.+\prod_{i=1}^{n}\left(1-b_{i}\right)\left[\left(\frac{1}{1+e^{g_{i} \gamma}}\right) \frac{e^{y_{i} x_{i} \beta}}{\left(1+e^{x_{i} \beta}\right)^{m_{i}}}\right]^{\hat{\phi}^{-1}}\right\} .
\end{aligned}
$$

Nota-se, através das expressões (31) e (32), que o parâmetro de dispersão é considerado apenas para $Y_{i}>0$, pois esse ocorre apenas para o caso em que pelo menos um ovo foi parasitado, dentre os 128 ovos.

A expressão do DIC usada para escolher o polinômio do modelo de regressão que melhor se ajusta aos dados é dada por

$$
D I C=\bar{D}(\boldsymbol{p}, \boldsymbol{w}, \phi ; \boldsymbol{y}, \boldsymbol{b})+p_{D}
$$

em que o logaritmo da função de verossimilhança é dado por

$$
\begin{aligned}
l(\boldsymbol{w}, \boldsymbol{p}, \phi ; \boldsymbol{y}, \boldsymbol{b}, \boldsymbol{m}) & =\log \left(L \prod_{i=1}^{n}\left(y_{i}, b_{i}, w_{i}, p_{i}, \phi, m_{i}\right)\right) \\
& \propto \frac{1}{\phi}\left\{\sum_{i=1}^{n}\left[b_{i} \log \left(\frac{w_{i}}{1-w_{i}}\right) \log \left(1-w_{i}\right)\right]\right. \\
& \left.+\sum_{i=1}^{n}\left(1-b_{i}\right)\left[y_{i} \log \left(\frac{p_{i}}{1-p_{i}}\right)+m_{i} \log \left(1-p_{i}\right)\right]\right\},
\end{aligned}
$$

e, portanto,

$$
\begin{aligned}
D(\boldsymbol{p}, \boldsymbol{w}, \phi ; \boldsymbol{y}, \boldsymbol{b}) & \propto \frac{-2}{\phi}\left\{\sum_{i=1}^{n}\left[b_{i} \log \left(\frac{w_{i}}{1-w_{i}}\right) \log \left(1-w_{i}\right)\right]\right. \\
& \left.+\sum_{i=1}^{n}\left(1-b_{i}\right)\left[y_{i} \log \left(\frac{p_{i}}{1-p_{i}}\right)+m_{i} \log \left(1-p_{i}\right)\right]\right\} .
\end{aligned}
$$

Logo,

$$
\begin{aligned}
\bar{D}(\boldsymbol{p}, \boldsymbol{w}, \phi ; \boldsymbol{y}, \boldsymbol{b}) & =E_{p \mid y}[D(\boldsymbol{p}, \boldsymbol{w}, \phi ; \boldsymbol{y}, \boldsymbol{b})]=E_{p \mid y}\left\{\frac { - 2 } { \phi } \left\{\sum_{i=1}^{n}\left[b_{i} \log \left(\frac{w_{i}}{1-w_{i}}\right) \log \left(1-w_{i}\right)\right]\right.\right. \\
& \left.\left.+\sum_{i=1}^{n}\left(1-b_{i}\right)\left[y_{i} \log \left(\frac{p_{i}}{1-p_{i}}\right)+m_{i} \log \left(1-p_{i}\right)\right]\right\}\right\}
\end{aligned}
$$




$$
\begin{aligned}
D(\overline{\boldsymbol{p}}, \overline{\boldsymbol{w}}, \phi ; \boldsymbol{y}, \boldsymbol{b}) & =\frac{-2}{\phi}\left\{\sum_{i=1}^{n}\left[b_{i} \log \left(\frac{\bar{w}_{i}}{1-\bar{w}_{i}}\right) \log \left(1-\bar{w}_{i}\right)\right]\right. \\
& \left.+\sum_{i=1}^{n}\left(1-b_{i}\right)\left[y_{i} \log \left(\frac{\bar{p}_{i}}{1-\bar{p}_{i}}\right)+m_{i} \log \left(1-\bar{p}_{i}\right)\right]\right\}
\end{aligned}
$$

e

$$
\begin{aligned}
D I C & =E_{p \mid y}\left\{\frac { - 2 } { \phi } \left\{\sum_{i=1}^{n}\left[b_{i} \log \left(\frac{w_{i}}{1-w_{i}}\right) \log \left(1-w_{i}\right)\right]\right.\right. \\
& \left.\left.+\sum_{i=1}^{n}\left(1-b_{i}\right)\left[y_{i} \log \left(\frac{p_{i}}{1-p_{i}}\right)+m_{i} \log \left(1-p_{i}\right)\right]\right\}\right\}+p_{D}
\end{aligned}
$$

em que $p_{i}=\frac{e^{x_{i} \beta}}{1+e^{x_{i} \beta}}, w_{i}=\frac{e^{g_{i} \gamma}}{1+e^{g_{i} \gamma}}$ e $p_{D}=\bar{D}(\boldsymbol{p}, \boldsymbol{w}, \phi ; \boldsymbol{y}, \boldsymbol{b})-D(\overline{\boldsymbol{p}}, \overline{\boldsymbol{w}}, \phi ; \boldsymbol{y}, \boldsymbol{b})$.

A verossimilhança marginal, necessária para obter o fator de Bayes, para o modelo ZIB é dada por,

$$
\begin{aligned}
\hat{\pi}(\boldsymbol{y} \mid M)=\frac{1}{T} & \sum_{t=1}^{T}\left\{\prod_{i=1}^{n} b_{i}\left[\frac{1}{1+e^{g_{i} \gamma^{(t)}}}\left(e^{g_{i} \gamma^{(t)}}+\frac{1}{\left(1+e^{x_{i} \beta^{(t)}}\right)^{m_{i}}}\right)\right]\right\}+ \\
& +\frac{1}{T} \sum_{t=1}^{T}\left\{\prod_{i=1}^{n}\left(1-b_{i}\right)\left[\left(\frac{1}{1+e^{g_{i} \gamma^{(t)}}}\right) \frac{e^{y_{i} x_{i} \beta^{(t)}}}{\left(1+e^{x_{i} \beta^{(t)}}\right)^{m_{i}}}\right]\right\},
\end{aligned}
$$

sendo $M$ o modelo corrente e $T$ o tamanho da amostra obtida pelo método MCMC. 


\section{RESULTADOS E DISCUSSÃO}

A seguir são apresentados os resultados obtidos para os modelos binomial superdisperso, binomial truncado superdisperso e ZIB superdisperso, discutidos em (3.2) e ajustados aos dados apresentados no Anexo A do ensaio de controle biológico.

\subsection{Modelo binomial superdisperso}

O ajuste do modelo maximal, considerando o número de fêmeas como variável qualitativa, teve como resultado uma deviance residual igual a 4.045,87 com 63 graus de liberdade, mostrando que existe variabilidade maior do que aquela esperada pelo modelo binomial padrão. Utilizando-se métodos MCMC ajustou-se o modelo com superdispersão constante e verificou-se que $\hat{\hat{\phi}}=57,063$. Observou-se que a convergência de $\phi$ foi satisfeita, através dos testes de diagnósticos de Raftery \& Lewis e de Heidelberger \& Welch e usando a análise gráfica dos valores gerados e da aproximação da densidade a posteriori condicional, apresentada na Figura 2.
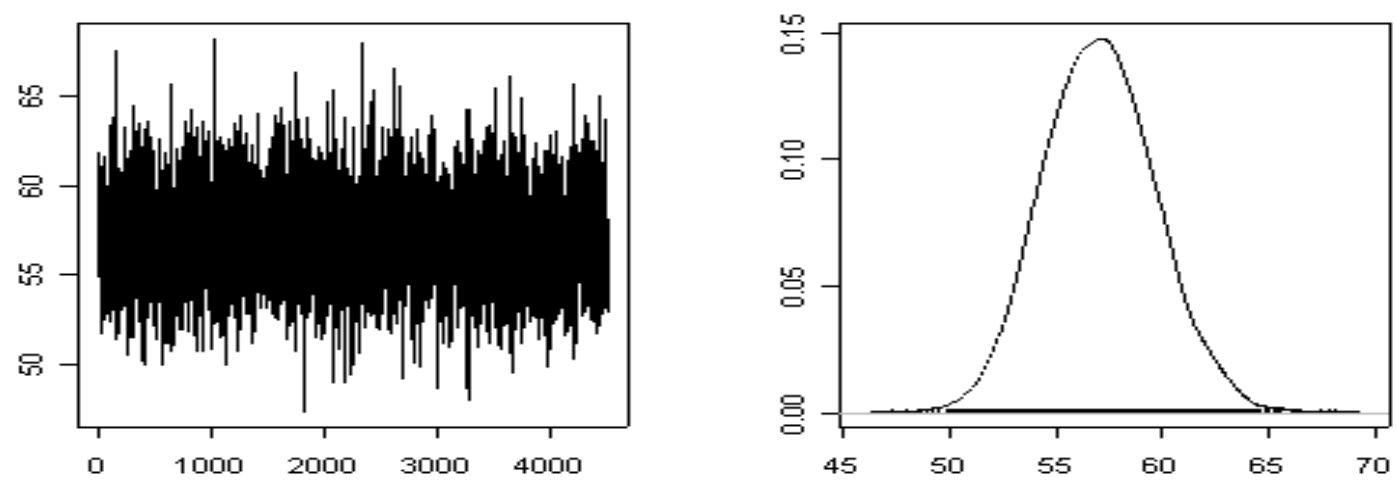

Figura 2 - Trajetória da cadeia ao longo das iterações e a aproximação da densidade a posteriori de $\phi$ para o modelo binomial 
Na Tabela 2 podem ser vistos os resultados obtidos para o DIC e para o número efetivo estimado de parâmetros (Spiegelhalter, 1998) para os diversos modelos ajustados aos dados do ensaio do controle biológico, considerando-se a distribuição binomial e como preditores lineares polinômios até o quarto grau, usando-se Metropolis-Hastings.

Tabela 2. Valores do DIC e respectivos números de parâmetros efetivos estimados para o modelo binomial

\begin{tabular}{lcc}
\hline Modelos & DIC & $p_{D}$ \\
\hline$\eta_{i}=\log \left(\frac{p_{i}}{1-p_{i}}\right)=\beta_{0}+\beta_{1} x_{i}$ & $4.538,610$ & 2,003 \\
$\eta_{i}=\log \left(\frac{p_{i}}{1-p_{i}}\right)=\beta_{0}+\beta_{1} x_{i}+\beta_{2} x_{i}^{2}$ & $4.538,570$ & 3,004 \\
$\eta_{i}=\log \left(\frac{p_{i}}{1-p_{i}}\right)=\beta_{0}+\beta_{1} x_{i}+\beta_{2} x_{i}^{2}+\beta_{3} x_{i}^{3}$ & & 4,104 \\
$\eta_{i}=\log \left(\frac{p_{i}}{1-p_{i}}\right)=\beta_{0}+\beta_{1} x_{i}+\beta_{2} x_{i}^{2}+\beta_{3} x_{i}^{3}+\beta_{4} x_{i}^{4}$ & $4.301,870$ & 8,403
\end{tabular}

Verifica-se que o valor do DIC é sempre decrescente. Observa-se, ainda, que o valor para o número efetivo de parâmetros $\left(p_{D}\right)$ no caso de polinômio de quarto grau é maior $(8,403)$ do que o número de parâmetros (5). Entretanto, Spiegelhalter (1998) observa que quanto maior o número de parâmetros no modelo, menos precisa pode-se tornar a estimativa do número efetivo de parâmetros. Além disso, salienta-se que não foi possível obter convergência para modelos com polinômio de grau maior do que 4. Na análise clássica desse conjunto de dados, Vieira (1998) verificou que o polinômio de terceiro grau ajustou-se melhor aos dados.

Uma outra forma de verificar o preditor linear para o modelo binomial é através do fator de Bayes, o qual utiliza a verossimilhança marginal da distribuição, apresentada na Tabela 3.

Comparando as estimativas da verossimilhança marginal encontradas 
Tabela 3. Valores da verossimilhança marginal para o modelo binomial

\begin{tabular}{lc}
\hline Modelos & $\hat{\pi}(y \mid M)$ \\
\hline$\eta_{i}=\beta_{0}+\beta_{1} x_{i}$ & $1,45.10^{-275}$ \\
$\eta_{i}=\beta_{0}+\beta_{1} x_{i}+\beta_{2} x_{i}^{2}$ & $1,53.10^{-281}$ \\
$\eta_{i}=\beta_{0}+\beta_{1} x_{i}+\beta_{2} x_{i}^{2}+\beta_{3} x_{i}^{3}$ & $3,49.10^{-282}$ \\
$\eta_{i}=\beta_{0}+\beta_{1} x_{i}+\beta_{2} x_{i}^{2}+\beta_{3} x_{i}^{3}+\beta_{4} x_{i}^{4}$ & $1,14.10^{-259}$ \\
\hline
\end{tabular}

para os preditores lineares do modelo binomial, verifica-se que o modelo com preditor linear de quarto grau apresentou maior valor da verossimilhança marginal, indicando que esse modelo ajusta-se melhor aos dados. Se os modelos polinomiais forem comparados dois a dois, notou-se que a razão do polinômio de quarto grau em relação aos demais polinômios é superior a 50. De acordo com a Tabela 1, existe uma forte evidência de que o modelo com polinômio de quarto grau ajusta-se melhor aos dados.

Ajustando a distribuição a posteriori condicional completa, dada em (21), obtiveram-se as estimativas a posteriori das médias, erros-padrões para $\hat{\beta}_{j}$ e intervalos de credibilidade para $\beta_{j}$ a $95 \%$ de probabilidade do modelo binomial superdisperso tendo como preditor linear um polinômio de quatro grau, conforme apresentados na Tabela 4.

Tabela 4. Sumários a posteriori do modelo binomial superdisperso, considerando como preditor linear como um polinômio de quarto grau

\begin{tabular}{ccccc}
\hline & & & \multicolumn{2}{c}{ Intervalo de credibilidade } \\
\cline { 4 - 5 } Parâmetros & Médias & Erro Padrão & $2,5 \%$ & $97,5 \%$ \\
\hline$\beta_{0}$ & $-1,4170$ & 0,2081 & $-1,8292$ & $-1,0203$ \\
$\beta_{1}$ & $-8,4476$ & 0,3055 & $-9,0577$ & $-7,8424$ \\
$\beta_{2}$ & 3,6147 & 0,0542 & 3,5079 & 3,7268 \\
$\beta_{3}$ & $-0,5806$ & 0,0063 & $-0,5940$ & $-0,5680$ \\
$\beta_{4}$ & 0,0318 & 0,0007 & 0,0303 & 0,0333 \\
\hline
\end{tabular}


Tem-se, então, que

$$
g\left(p_{i}\right)=\log \left(\frac{p_{i}}{1-p_{i}}\right)=-1,417-8,4476 x_{i}+3,6147 x_{i}^{2}-0,5806 x_{i}^{3}+0,0318 x_{i}^{4} .
$$

Observando o gráfico dos valores preditos versus o logaritmo do número de fêmeas (Figura 3), usando como preditor linear o polinômio de quarto grau, vêse que os valores observados estão dentro do intervalo de credibilidade de $95 \%$ de probabilidade.

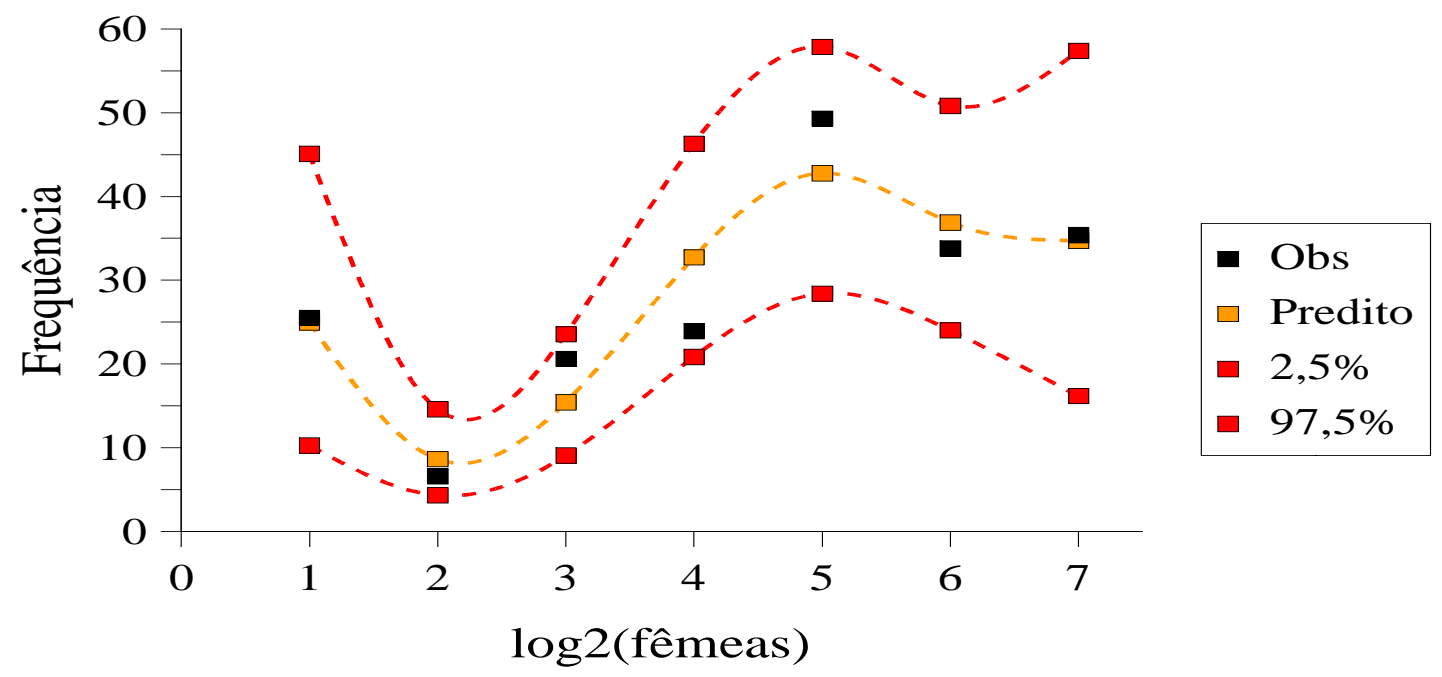

Figura 3 - Valores observados, preditos e intervalos de credibilidade a $95 \%$ de probabilidade para o modelo binomial superdisperso considerando o preditor linear como um polinômio de quarto grau

Verificou-se, ainda, que as estimativas de $\beta_{j}$, para $j=0,1,2,3,4$ tiveram uma taxa de aceitação, em torno de 30\% para o algoritmo MetropolisHastings. Além disso, através da análise gráfica dos valores gerados de $\beta_{j}$ e usando os diagnósticos de Raftery \& Lewis e de Heidelberger \& Welch, implementado no módulo CODA do programa $\mathrm{R}$, foi observado que as condições para convergência de $\beta_{j}$ foram satisfeitas. Os valores gerados de $\beta_{j}$ ao longo das 4.500 iterações dos parâmetros e as aproximações das densidades a posteriori condicionais de $\beta_{0}, \beta_{1}, \beta_{2}$, 
$\beta_{3}$ e $\beta_{4}$ estão apresentadas nas Figuras 4, 5, 6, 7 e 8 respectivamente, observando-se que as diferentes cadeias se aproximam de um valor constante ao longo das iterações e as densidades a posteriori condicionais apresentam a forma da distribuição normal, tendo assim, indicativos de convergência.
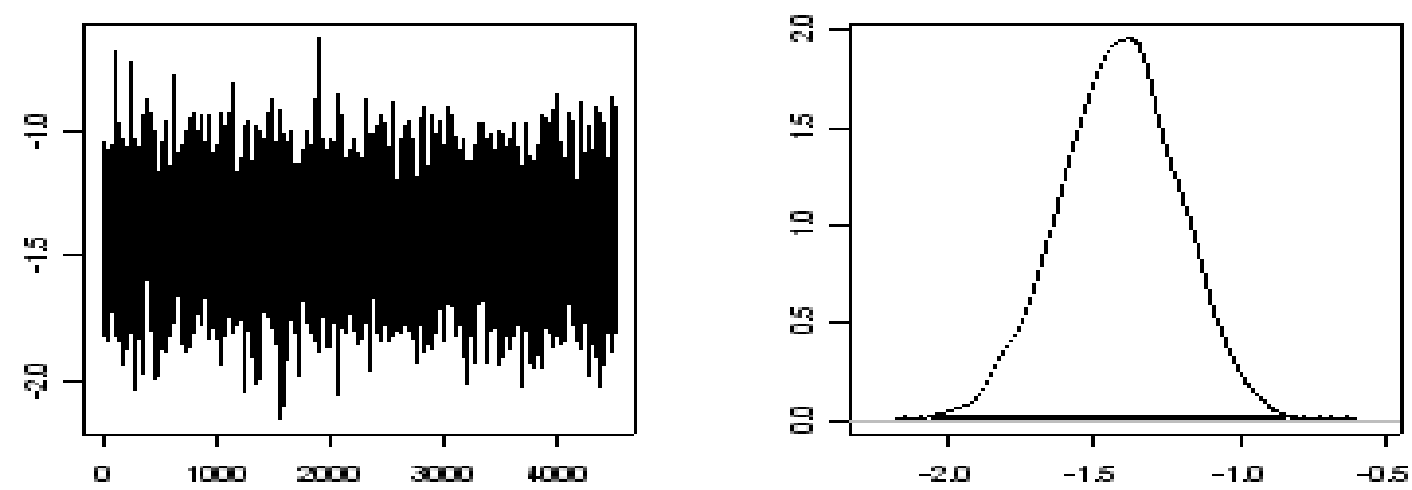

Figura 4 - Trajetória da cadeia ao longo das iterações e a aproximação da densidade a posteriori de $\beta_{0}$ para o modelo binomial
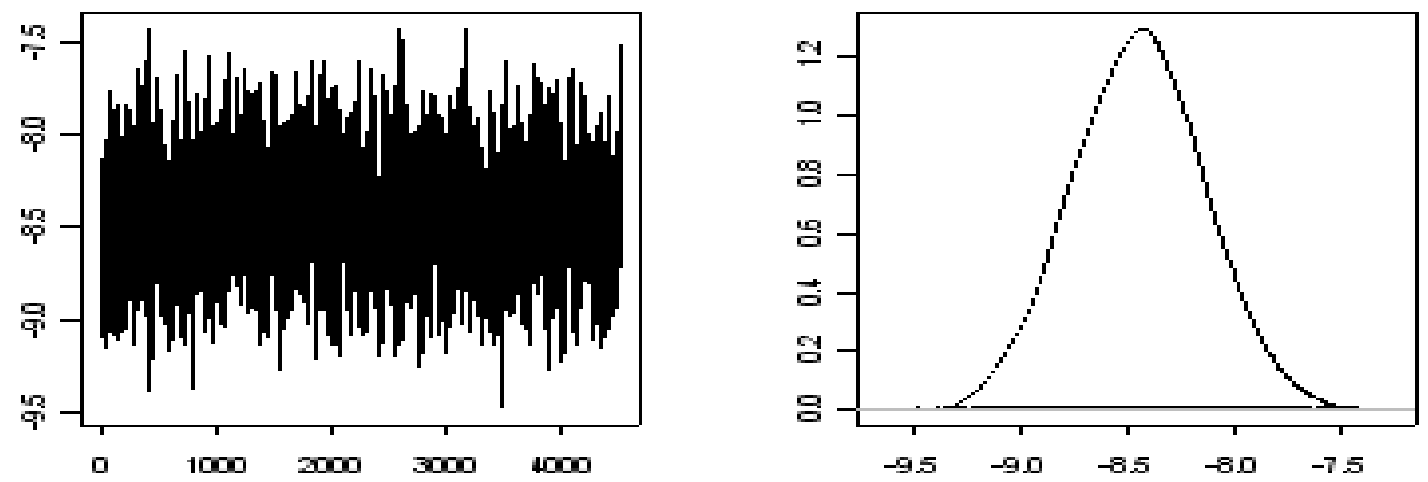

Figura 5 - Trajetória da cadeia ao longo das iterações e a aproximação da densidade a posteriori de $\beta_{1}$ para o modelo binomial 

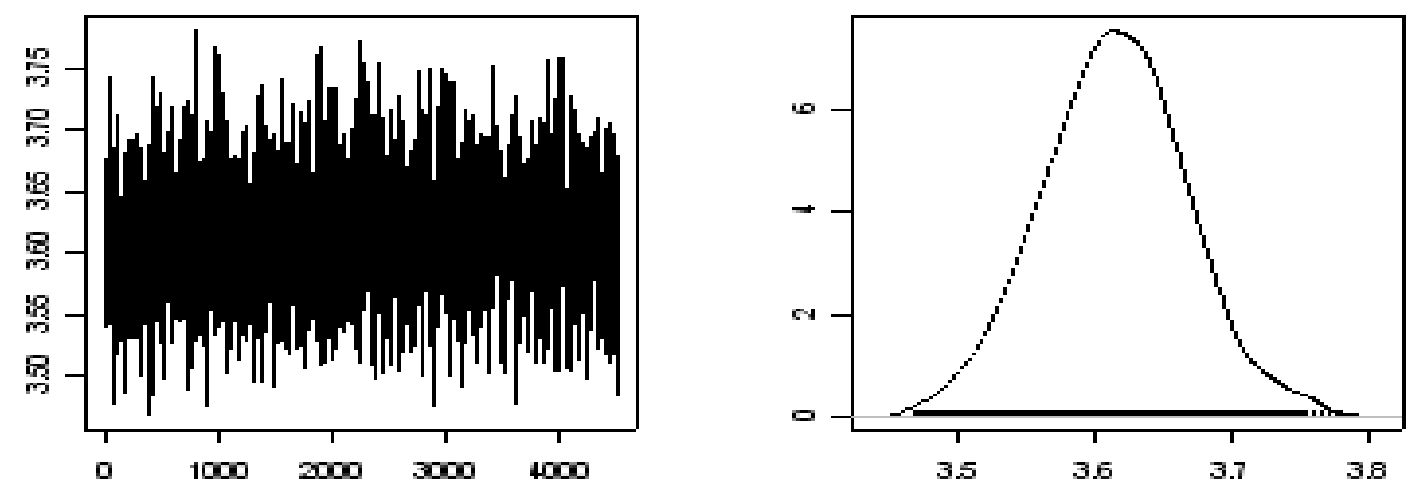

Figura 6 - Trajetória da cadeia ao longo das iterações e a aproximação da densidade a posteriori de $\beta_{2}$ para o modelo binomial
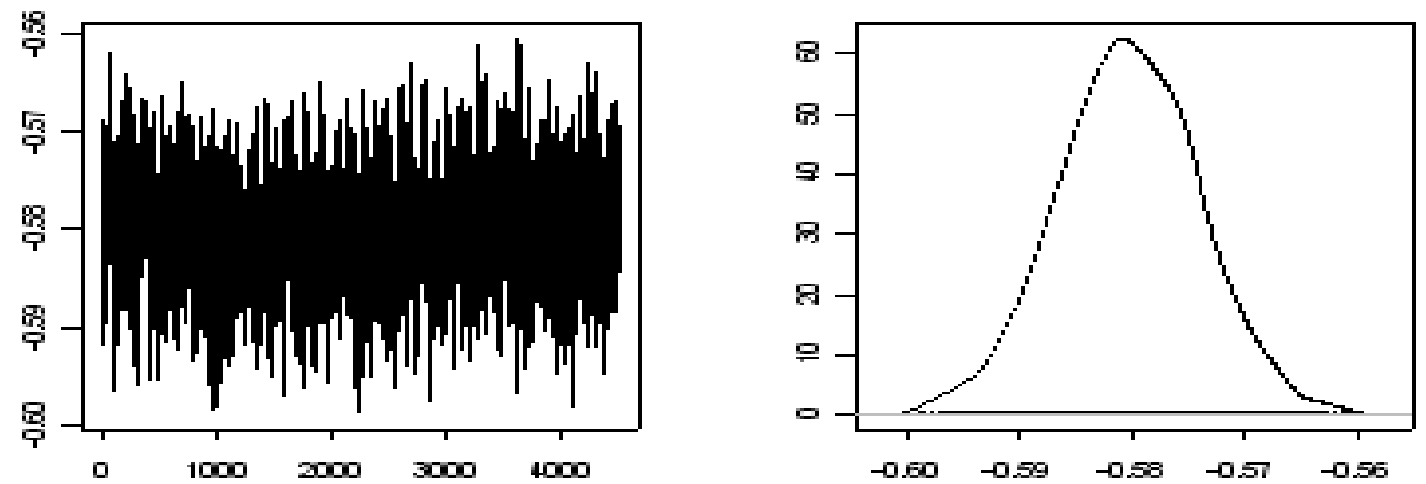

Figura 7 - Trajetória da cadeia ao longo das iterações e a aproximação da densidade a posteriori de $\beta_{3}$ para o modelo binomial
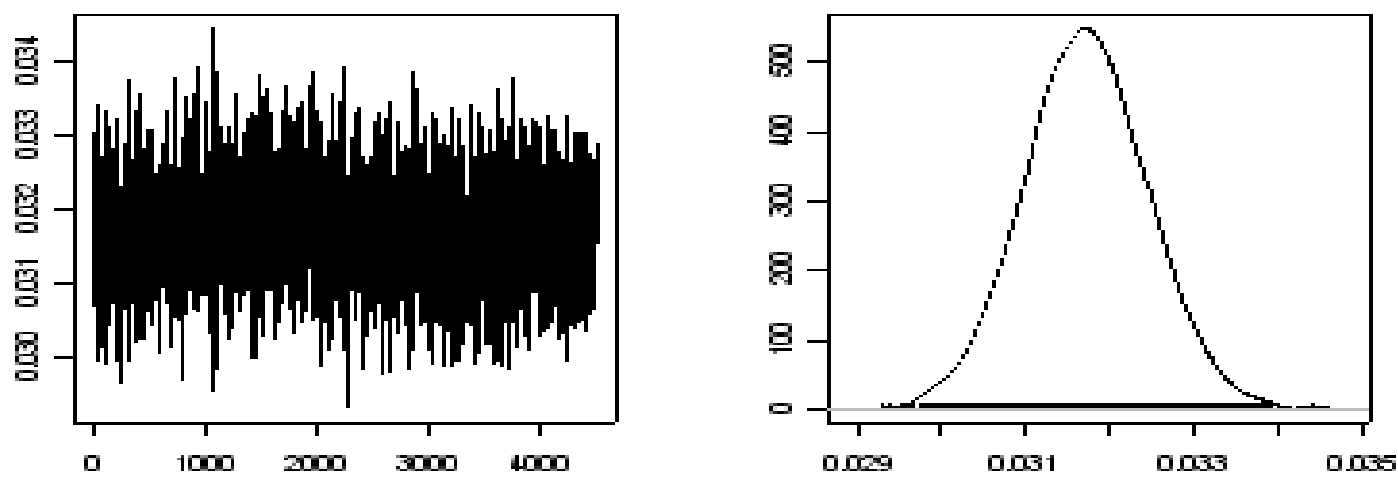

Figura 8 - Trajetória da cadeia ao longo das iterações e a aproximação da densidade a posteriori de $\beta_{4}$ para o modelo binomial 


\subsection{Modelo binomial truncado superdisperso}

O ajuste do modelo maximal teve como resultado uma deviance residual igual a 463, 2 com 26 graus de liberdade, mostrando que a variabilidade dos dados é maior do que aquela esperada pelo modelo binomial truncado. Usando o modelo binomial truncado superdisperso, com variância dada em (22), obteve-se através dos métodos MCMC a estimativa $\hat{\hat{\phi}}=19,465$. Verificou-se que a convergência de $\phi$ foi satisfeita usando os testes de diagnósticos de Raftery \& Lewis e Heidelberger \& Welch e através da análise gráfica dos valores gerados e da aproximação da densidade a posteriori condicional, apresentadas na Figura 9.
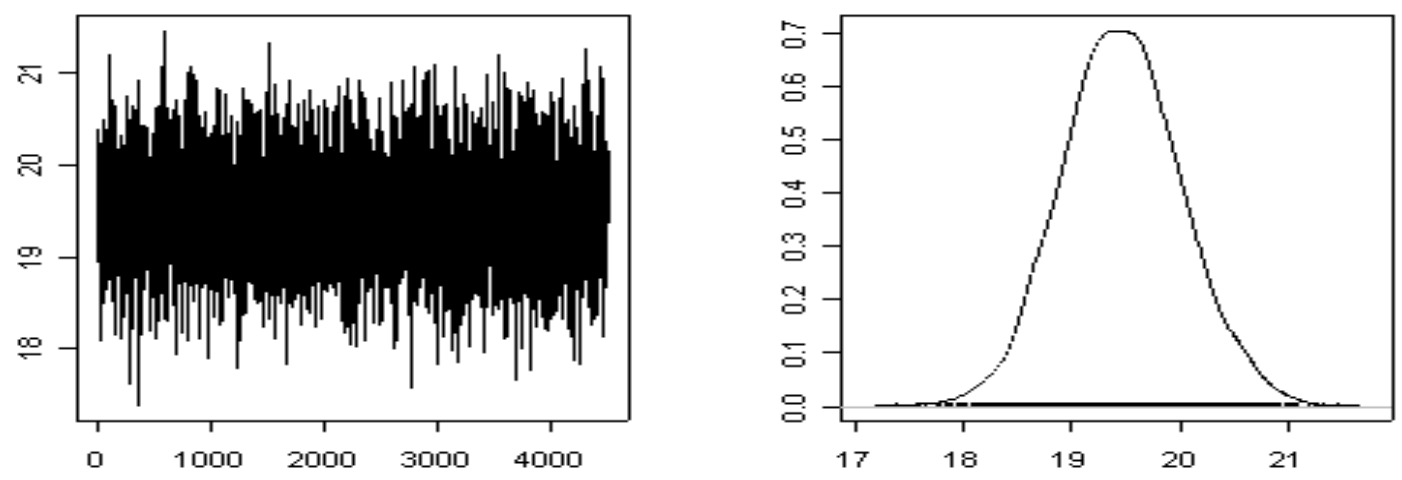

Figura 9 - Trajetória da cadeia ao longo das iterações e a aproximação da densidade a posteriori de $\phi$ para o modelo binomial truncado

Na Tabela 5 podem ser vistos os resultados obtidos para o DIC e para o número efetivo de parâmetros dos diversos modelos polinomiais ajustados considerando-se a distribuição binomial truncada e como preditores lineares polinômios até o quarto grau, usando-se Metropolis-Hastings. É importante salientar que para o ajuste desses modelos, supõe-se que o pesquisador não tem interesse nos ovos que não foram parasitados, com isso as observações nulas foram eliminadas do modelo.

Nota-se que o menor valor do DIC foi dado pelo modelo polinomial de terceiro grau. Observa-se, ainda, que a exemplo do modelo binomial, não foi possível obter convergência para modelos com polinômios de grau maior do que 4. Vieira 
Tabela 5. Valores do DIC e respectivos números efetivos de parâmetros estimados para o modelo binomial truncado

\begin{tabular}{lcc}
\hline Modelos & DIC & $p_{D}$ \\
\hline$\eta_{i}=\log \left(\frac{p_{i}}{1-p_{i}}\right)=\beta_{0}+\beta_{1} x_{i}$ & $1.027,926$ & 2,007 \\
$\eta_{i}=\log \left(\frac{p_{i}}{1-p_{i}}\right)=\beta_{0}+\beta_{1} x_{i}+\beta_{2} x_{i}^{2}$ & $1.016,992$ & 3,019 \\
$\eta_{i}=\log \left(\frac{p_{i}}{1-p_{i}}\right)=\beta_{0}+\beta_{1} x_{i}+\beta_{2} x_{i}^{2}+\beta_{3} x_{i}^{3}$ & 704,697 & 4,025 \\
$\eta_{i}=\log \left(\frac{p_{i}}{1-p_{i}}\right)=\beta_{0}+\beta_{1} x_{i}+\beta_{2} x_{i}^{2}+\beta_{3} x_{i}^{3}+\beta_{4} x_{i}^{4}$ & $1.062,138$ & 4,589 \\
\hline
\end{tabular}

(1998) observou, usando a análise clássica dos dados, que o polinômio de terceiro grau para o modelo truncado superdisperso, ajustou-se melhor aos dados.

Na Tabela 6 podem ser encontrados os valores da verossimilhança marginal para os diversos modelos polinomiais ajustados com preditores lineares, polinômios de até quarto grau.

Tabela 6. Valores da verossimilhança marginal para o modelo binomial truncado

\begin{tabular}{lc}
\hline Modelos & $\hat{\pi}(y \mid M)$ \\
\hline$\eta_{i}=\beta_{0}+\beta_{1} x_{i}$ & $8,05.10^{-81}$ \\
$\eta_{i}=\beta_{0}+\beta_{1} x_{i}+\beta_{2} x_{i}^{2}$ & $1,97.10^{-81}$ \\
$\eta_{i}=\beta_{0}+\beta_{1} x_{i}+\beta_{2} x_{i}^{2}+\beta_{3} x_{i}^{3}$ & $1,117.10^{-62}$ \\
$\eta_{i}=\beta_{0}+\beta_{1} x_{i}+\beta_{2} x_{i}^{2}+\beta_{3} x_{i}^{3}+\beta_{4} x_{i}^{4}$ & $1,08.10^{-62}$ \\
\hline
\end{tabular}

Comparando as estimativas da verossimilhança marginal dos preditores lineares do modelo binomial truncado, verificou-se que os modelos com polinômios de terceiro e de quarto graus tiveram estimativas próximas uma da outra, sendo a razão de 1,03 , tendo indicativos de que esses modelos tiveram comportamento semelhante 
no ajuste dos dados e melhores do que os demais modelos polinomiais. Portanto, para o ajuste do modelo escolhe-se aquele com menor número de parâmetros, ou seja, opta-se pelo modelo com preditor linear de terceiro grau. É importante observar que usando o DIC o modelo com polinômio de quarto grau é menos eficiente do que o modelo com polinômio de terceiro grau, entretanto esse resultado não ficou caracterizado usando o fator de Bayes.

Ajustando a distribuição a posteriori condicional completa, dada em (24), obtiveram-se as estimativas a posteriori das médias, erros-padrões para $\hat{\beta}_{j}$ e intervalos de credibilidade de $\beta_{j}$ a $95 \%$ de probabilidade para o modelo binomial truncado superdisperso, tendo como preditor linear um polinômio de terceiro grau, conforme apresentados na Tabela 7.

Tabela 7. Sumários a posteriori do modelo binomial truncado superdisperso, considerando o preditor linear como um polinômio de terceiro grau

\begin{tabular}{ccccc}
\hline & & & \multicolumn{2}{c}{ Intervalo de credibilidade } \\
\cline { 4 - 5 } Parâmetros & Médias & Erro Padrão & $2,5 \%$ & $97,5 \%$ \\
\hline$\beta_{0}$ & $-0,1716$ & 0,1426 & $-0,4633$ & 0,1051 \\
$\beta_{1}$ & $-3,8459$ & 0,2323 & $-4,2973$ & $-3,4027$ \\
$\beta_{2}$ & 1,2343 & 0,0453 & 1,1503 & 1,3292 \\
$\beta_{3}$ & $-0,1050$ & 0,0044 & $-0,1138$ & $-0,0965$ \\
\hline
\end{tabular}

Tem-se, então, que

$$
g\left(p_{i}\right)=\log \left(\frac{p_{i}}{1-p_{i}}\right)=-0,1716-3,8459 x_{i}+1,2343 x_{i}^{2}-0,105 x_{i}^{3} .
$$

Nota-se que o gráfico dos valores preditos versus o logaritmo do número de fêmeas (Figura 10), usando como preditor linear o polinômio de terceiro grau, vêse que os valores observados estão dentro do intervalo de credibilidade de $95 \%$ de probabilidade.

Observou-se que as estimativas de $\beta_{j}$, para $j=0,1,2,3$ tiveram uma taxa de aceitação em torno de $35 \%$ para o algoritmo Metropolis-Hastings através 


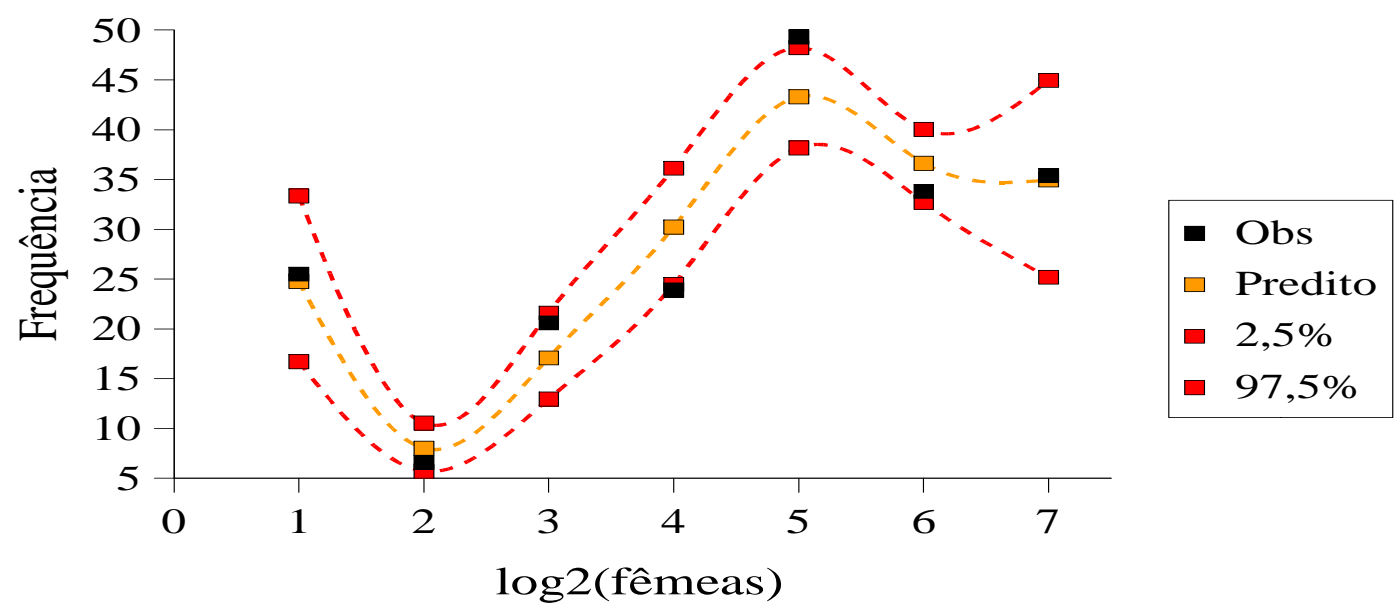

Figura 10 - Valores observados, preditos e intervalos de credibilidade a $95 \%$ de probabilidade para o modelo binomial truncado superdisperso considerando o preditor linear como um polinômio de terceiro grau

da análise gráfica dos valores gerados de $\beta_{j}$ e usando os testes de diagnósticos de Raftery \& Lewis e de Heidelberger \& Welch, implementado no módulo CODA do programa $\mathrm{R}$, notou-se que as condições para convergência de $\beta_{j}$ foram atendidas. As Figuras 11, 12, 13 e 14 apresentam os traços das cadeias ao longo das 4.500 iterações dos parâmetros e as aproximações das densidades a posteriori condicionais de $\beta_{0}$, $\beta_{1}, \beta_{2}$ e $\beta_{3}$ respectivamente, observando-se que os valores gerados aproximam-se de um valor constante e as densidades a posteriori condicionais apresentam a forma da distribuição normal, tendo assim, indicativos de convergência.
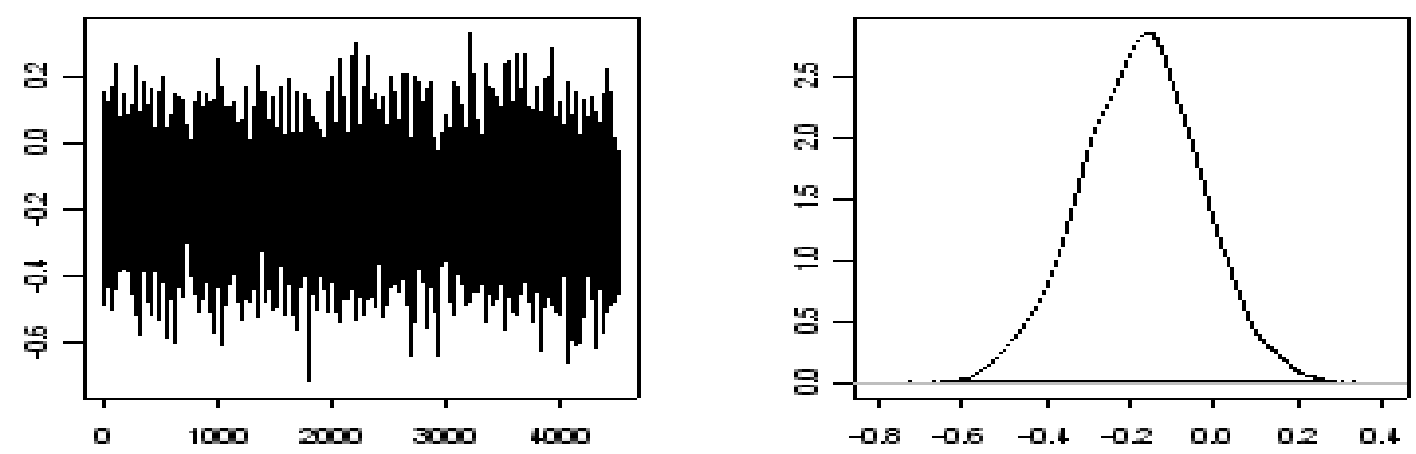

Figura 11 - Trajetória da cadeia ao longo das iterações e a aproximação da densidade a posteriori de $\beta_{0}$ para o modelo binomial truncado 

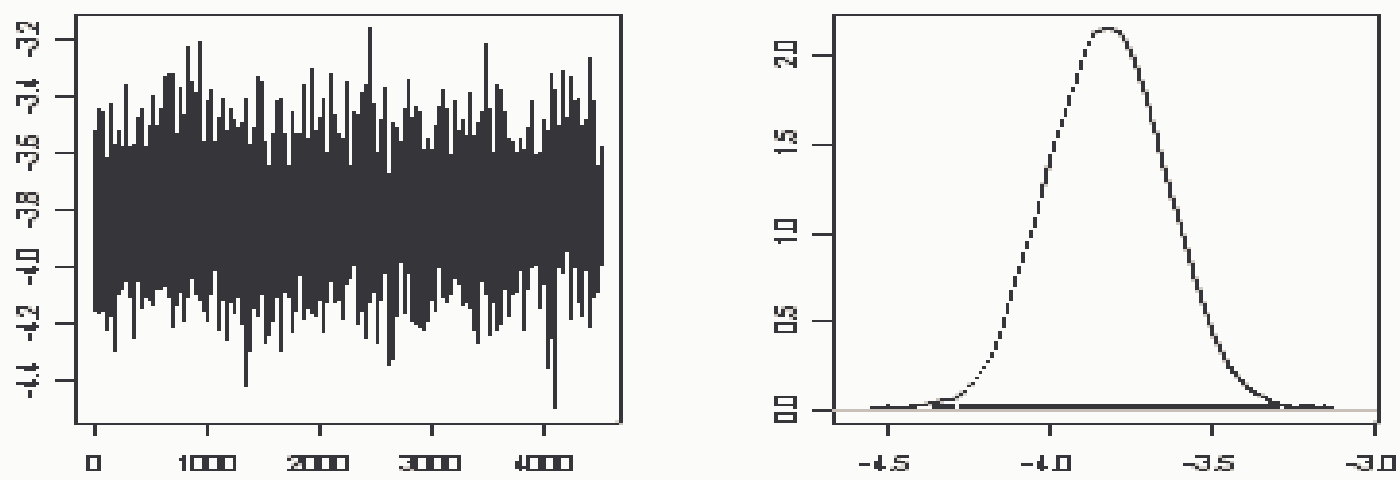

Figura 12 - Trajetória da cadeia ao longo das iterações e a aproximação da densidade a posteriori de $\beta_{1}$ para o modelo binomial truncado
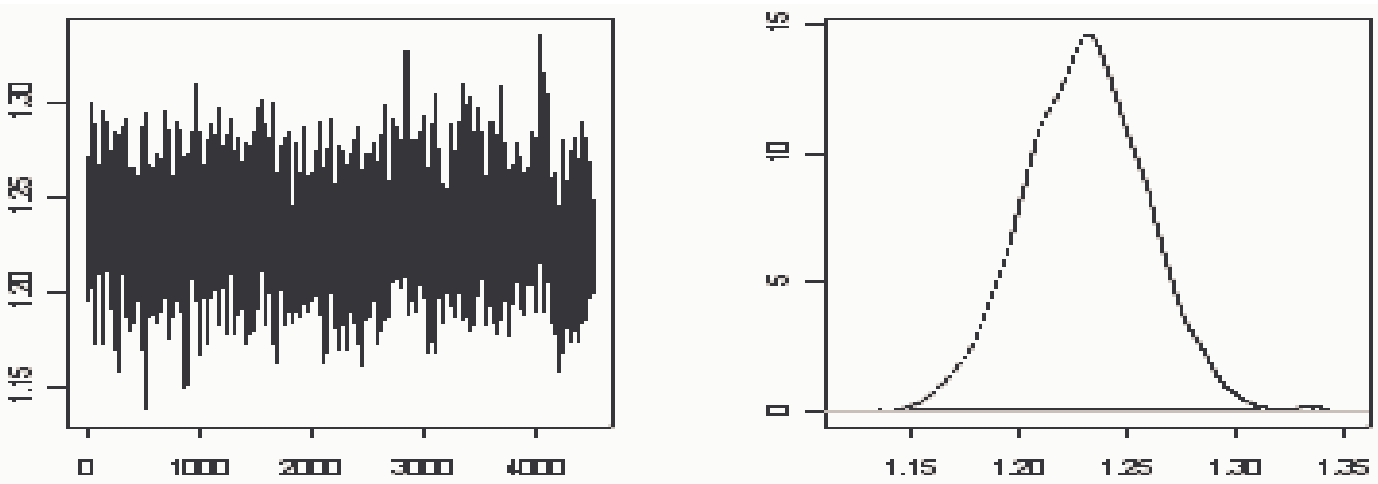

Figura 13 - Trajetória da cadeia ao longo das iterações e a aproximação da densidade a posteriori de $\beta_{2}$ para o modelo binomial truncado
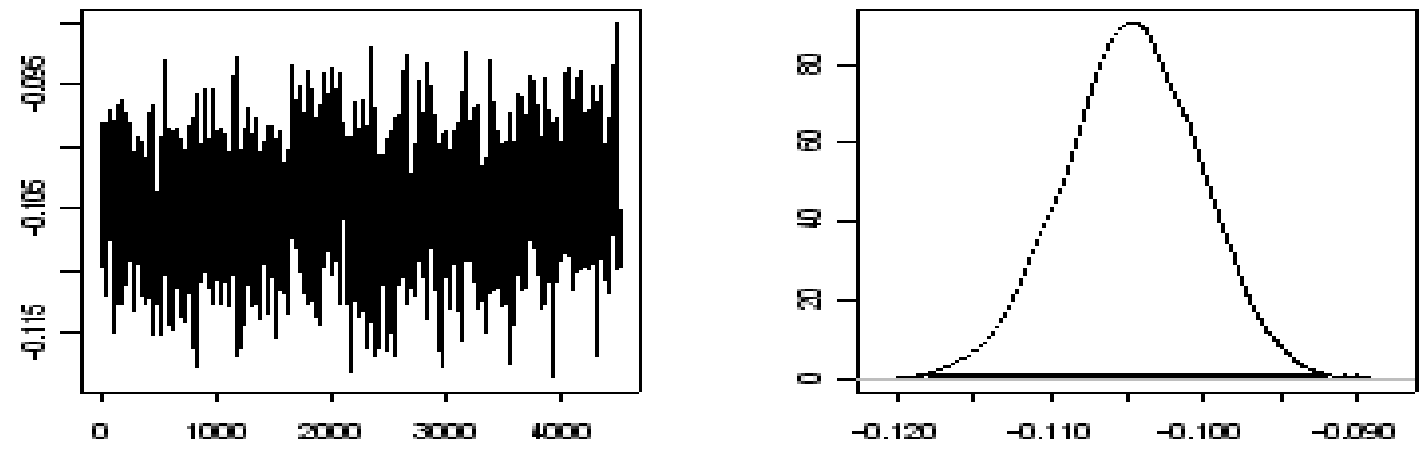

Figura 14 - Trajetória da cadeia ao longo das iterações e a aproximação da densidade a posteriori de $\beta_{3}$ para o modelo binomial truncado 


\subsection{Modelo binomial inflacionado de zeros superdisperso}

O ajuste do modelo maximal teve como resultado uma deviance residual igual a 461,2 com 62 graus de liberdade, mostrando que existe variabilidade maior do que a observada no modelo ZIB padrão.

Usando os métodos MCMC, estimou-se o valor do parâmetro de dispersão do modelo ZIB superdisperso, com variância dada em (26), e obteve-se $\hat{\hat{\phi}}=12,116$. Usando os testes de diagnósticos de Raftery \& Lewis e de Heidelberger \& Wech e a análise gráfica através dos valores gerados ao longo das iterações e da aproximação da densidade a posteriori condicional, apresentadas na Figura 15, verificou-se que a convergência de $\phi$ foi satisfeita.
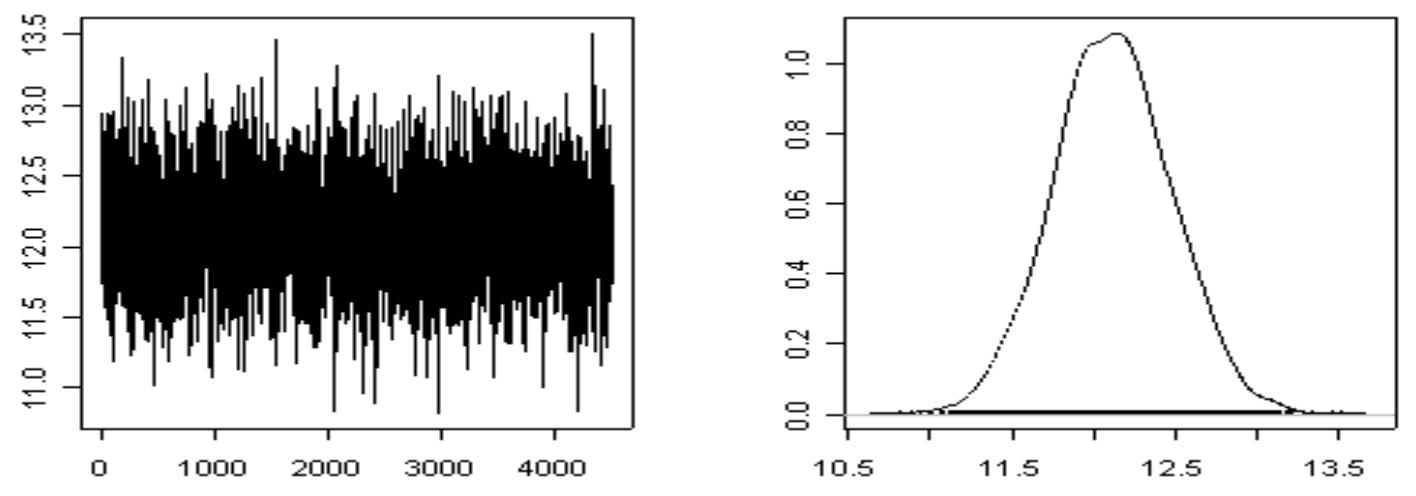

Figura 15 - Trajetória da cadeia ao longo das iterações e a aproximação da densidade a posteriori de $\phi$ para o modelo ZIB

Para o ajuste do modelo ZIB superdisperso, consideraram-se vários modelos polinomiais e observou-se que o preditor linear com o polinômio de terceiro grau para as proporções positivas $(\boldsymbol{p})$ foi o que melhor se ajustou aos dados. Portanto, para a escolha do polinômio para as proporções de zeros $(\boldsymbol{w})$ fixou-se $\boldsymbol{p}$.

Na Tabela 8 são apresentados os resultados obtidos para o DIC e para o número efetivo estimado de parâmetros para os modelos polinomiais considerando-se a distribuição binomial inflacionada de zeros e como preditores lineares, polinômios até o quarto grau para a proporção de zeros, $\boldsymbol{w}$ e fixando-se o polinômio de terceiro 
grau para as proporções positivas, $\boldsymbol{p}$. O programa usado para obter esses resultados foi implementado no WinBugs, sendo apresentado no Anexo B.

Tabela 8. Valores do DIC e respectivos números efetivos de parâmetros estimados para o modelo binomial inflacionado de zeros

\begin{tabular}{lcc}
\hline Modelos & DIC & $p_{D}$ \\
\hline Fixando-se: $\eta_{1 i}=\log \left(\frac{p_{i}}{1-p_{i}}\right)=\beta_{0}+\beta_{1} x_{i}+\beta_{2} x_{i}^{2}+\beta_{3} x_{i}^{3}$ & & \\
$\eta_{2 i}=\log \left(\frac{w_{i}}{1-w_{i}}\right)=\gamma_{0}$ & 803,7 & 5,135 \\
$\eta_{2 i}=\log \left(\frac{w_{i}}{1-w_{i}}\right)=\gamma_{0}+\gamma_{1} g_{i}$ & 805,1 & 6,01 \\
$\eta_{2 i}=\log \left(\frac{w_{i}}{1-w_{i}}\right)=\gamma_{0}+\gamma_{1} g_{i}+\gamma_{2} g_{i}^{2}$ & & \\
$\eta_{2 i}=\log \left(\frac{w_{i}}{1-w_{i}}\right)=\gamma_{0}+\gamma_{1} g_{i}+\gamma_{2} g_{i}^{2}+\gamma_{3} g_{i}^{3}$ & 807,5 & \\
$\eta_{2 i}=\log \left(\frac{w_{i}}{1-w_{i}}\right)=\gamma_{0}+\gamma_{1} g_{i}+\gamma_{2} g_{i}^{2}+\gamma_{3} g_{i}^{3}+\gamma_{4} g_{i}^{4}$ & 809,4 & \\
\end{tabular}

Verificou-se que o menor valor do DIC foi dado pelo modelo constante para $\boldsymbol{w}$ e pelo modelo de terceiro grau para $\boldsymbol{p}$. A exemplo dos modelos citados nas seções anteriores, os modelos de graus superiores a 4 apresentaram problemas de convergência.

Na Tabela 9 podem ser encontrados os valores da verossimilhança marginal para os modelos polinomiais considerando-se a distribuição ZIB e como preditores lineares, polinômios até o quarto grau para a proporção de zeros, $\boldsymbol{w}$ e fixando-se o polinômio de terceiro grau para as proporções positivas, $\boldsymbol{p}$.

Comparando as estimativas da verossimilhança marginal dos preditores lineares do modelo ZIB, verifica-se que todos os modelos apresentaram estimativas próximas uma da outra, sendo que a razão entre elas não é maior do que 3, indicando que esses modelos tiveram comportamento semelhante no ajuste dos dados. 
Tabela 9. Valores da verossimilhança marginal para o modelo ZIB

\begin{tabular}{ll}
\hline Modelos & $\hat{\pi}(y \mid M)$ \\
\hline Fixando-se: $\eta_{1}=\beta_{0}+\beta_{1} x_{i}+\beta_{2} x_{i}^{2}+\beta_{3} x_{i}^{3}$ & \\
$\eta_{2}=\gamma_{0}$ & $7,995.10^{-71}$ \\
$\eta_{2}=\gamma_{0}+\gamma_{1} x_{i}$ & $7,144.10^{-71}$ \\
$\eta_{2}=\gamma_{0}+\gamma_{1} x_{i}+\gamma_{2} x_{i}^{2}$ & $6,99.10^{-71}$ \\
$\eta_{2}=\gamma_{0}+\gamma_{1} x_{i}+\gamma_{2} x_{i}^{2}+\gamma_{3} x_{i}^{3}$ & $6,741.10^{-71}$ \\
$\eta_{2}=\gamma_{0}+\gamma_{1} x_{i}+\gamma_{2} x_{i}^{2}+\gamma_{3} x_{i}^{3}+\gamma_{4} x_{i}^{4}$ & $3,657.10^{-71}$ \\
\hline
\end{tabular}

Portanto, para o ajuste do modelo ZIB escolhe-se aquele com menor número de parâmetros, ou seja, opta-se pelo modelo com preditor linear de terceiro grau para as proporções positivas $\boldsymbol{p}$ e constante para $\boldsymbol{w}$.

Ajustando as distribuições a posteriori condicionais completas, dadas em (31) e (32), usando o programa apresentado no Anexo C, obtiveram-se estimativas a posteriori das médias, erros-padrões de $\hat{\beta}_{j}$ e intervalos de credibilidade de $\beta_{j}$ a $95 \%$ de probabilidade do modelo ZIB superdisperso tendo como preditores lineares os polinômios de terceiro grau para $\boldsymbol{p}$ e constante para $\boldsymbol{w}$, conforme apresentados na Tabela 10 .

Tabela 10. Sumários a posteriori do modelo binomial inflacionado de zeros superdisperso, considerando os preditors linear como um polinômio de terceiro grau para $\boldsymbol{p}$ e constante para $\boldsymbol{w}$

\begin{tabular}{ccccc}
\hline & & & \multicolumn{2}{c}{ Intervalo de credibilidade } \\
\cline { 4 - 5 } Parâmetros & Médias & Erro Padrão & $2,5 \%$ & $97,5 \%$ \\
\hline$\beta_{0}$ & $-0,2026$ & 0,1160 & $-0,4330$ & 0,0233 \\
$\beta_{1}$ & $-3,8362$ & 0,1976 & $-4,2113$ & $-3,4506$ \\
$\beta_{2}$ & 1,2312 & 0,0405 & 1,1495 & 1,3093 \\
$\beta_{3}$ & $-0,1047$ & 0,0039 & $-0,1123$ & $-0,0969$ \\
$\gamma_{0}$ & 0,1070 & 0,2345 & $-0,3501$ & 0,5661 \\
\hline
\end{tabular}


Tem-se, então, para as proporções positivas, que

$$
g\left(p_{i}\right)=\log \left(\frac{p_{i}}{1-p_{i}}\right)=-0,2026-3,8362 x_{i}+1,2312 x_{i}^{2}-0,1047 x_{i}^{3}
$$

e para as proporções de zeros

$$
g\left(w_{i}\right)=\log \left(\frac{w_{i}}{1-w_{i}}\right)=0,1070 .
$$

Fazendo o gráfico dos valores preditos versus o logaritmo do número de fêmeas (Figura 16), usando os preditores lineares como polinômios de terceiro grau para $\boldsymbol{p}$ e constante para $\boldsymbol{w}$, vê-se que os valores observados estão dentro do intervalo de credibilidade de $95 \%$ de probabilidade.

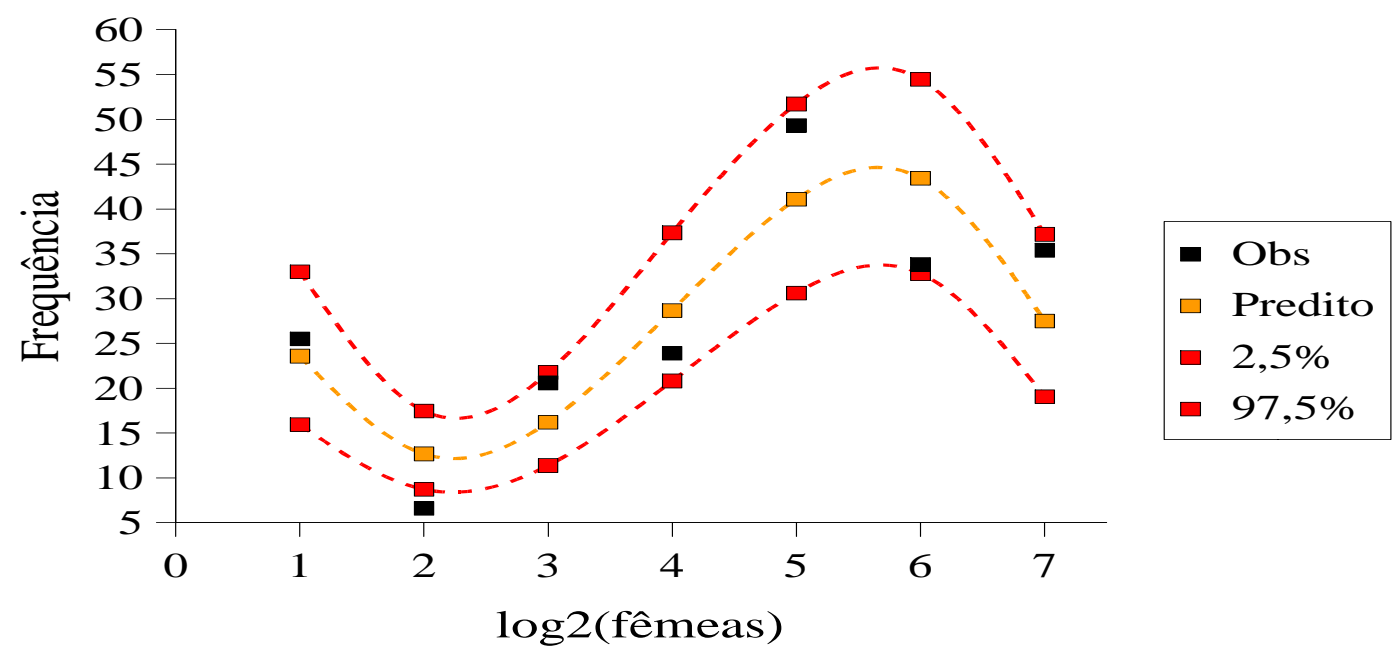

Figura 16 - Valores observados, preditos e intervalos de credibilidade a $95 \%$ de probabilidade para o modelo ZIB superdisperso considerando os preditores lineares como polinômios de terceiro grau para $\boldsymbol{p}$ e constante para $\boldsymbol{w}$

Verificou-se, ainda, que as estimativas de $\beta_{j}$, para $j=0,1,2,3$ e $\gamma_{0}$ tiveram uma taxa de aceitação variando de $30 \%$ a $40 \%$ para o algoritmo MetropolisHastings. Através da análise gráfica dos valores gerados de $\beta_{j}$ e $\gamma_{0}$ e dos testes de diagnósticos de Raftery \& Lewis e de Heidelberger \& Welch, observou-se que as condições para a convergência de $\beta_{j}$ e $\gamma_{0}$ foram satisfeitas. As Figuras 17, 18, 
19, 20 e 21 apresentam os traços das cadeias ao longo das iterações dos parâmetros e as aproximações das densidades a posteriori condicionais de $\beta_{0}, \beta_{1}, \beta_{2}, \beta_{3}$ e $\gamma_{0}$ respectivamente, notando-se que as diferentes cadeias se aproximam de um valor constante ao longo das iterações e as densidades a posteriori condicionais apresentam a forma da distribuição normal, tendo assim, indicativos de convergência.
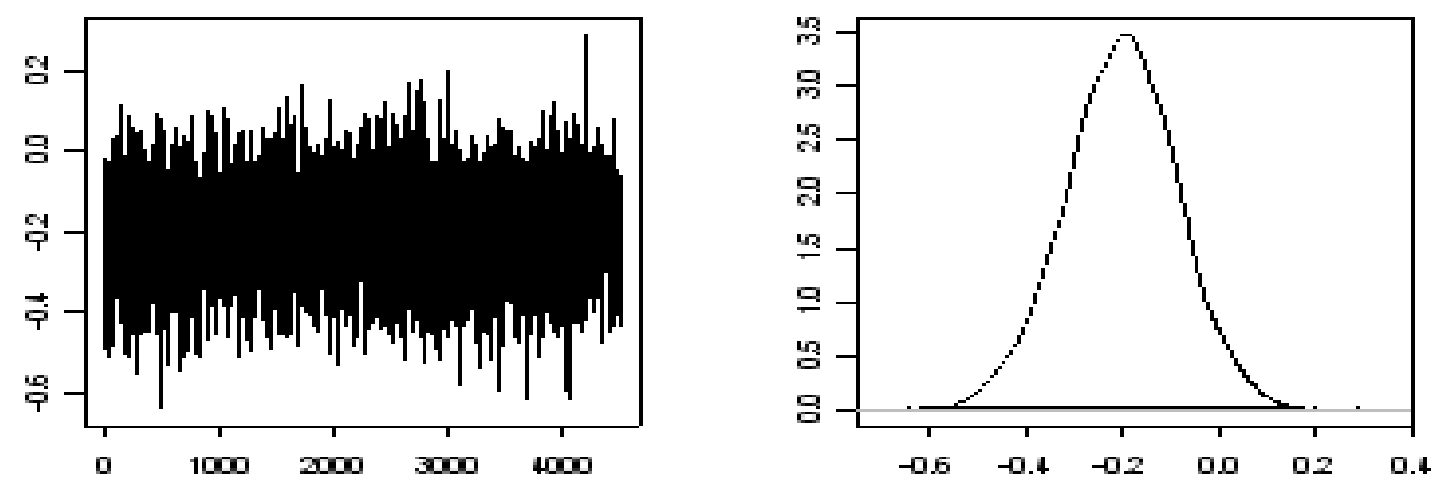

Figura 17 - Trajetória da cadeia ao longo das iterações e a aproximação da densidade a posteriori de $\beta_{0}$ para o modelo ZIB
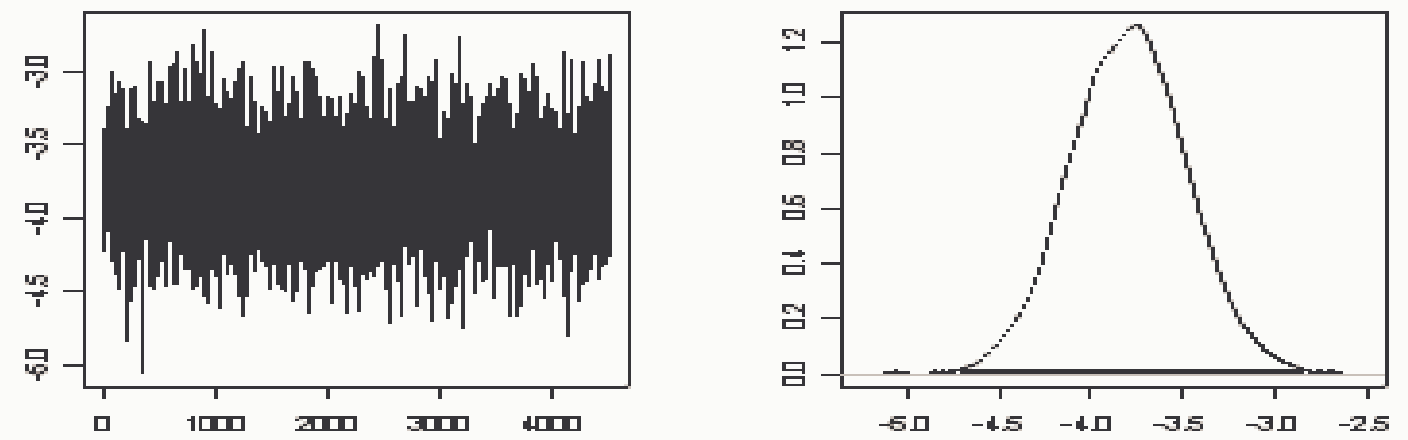

Figura 18 - Trajetória da cadeia ao longo das iterações e a aproximação da densidade a posteriori de $\beta_{1}$ para o modelo ZIB 

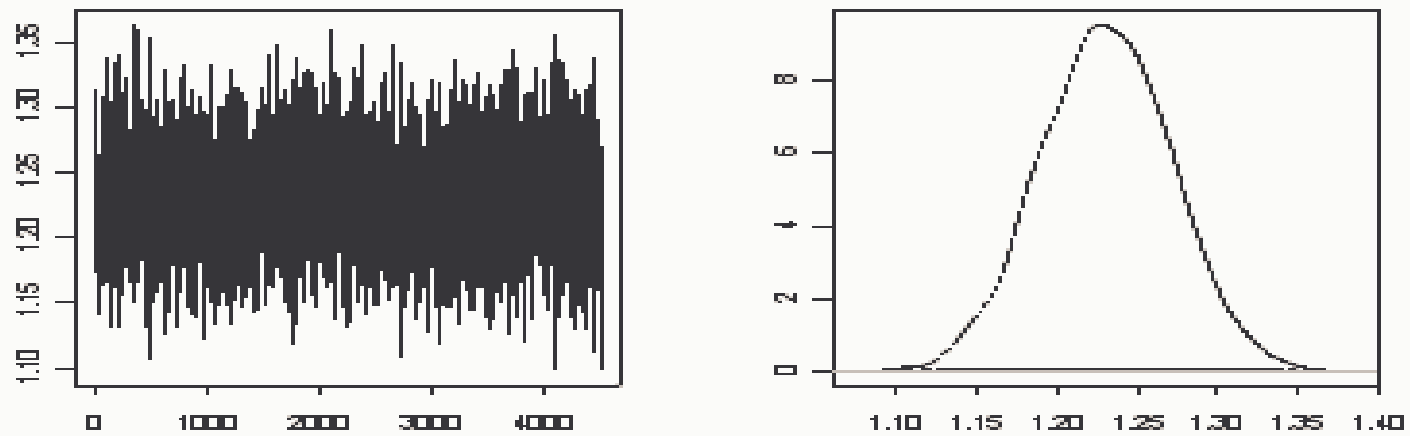

Figura 19 - Trajetória da cadeia ao longo das iterações e a aproximação da densidade a posteriori de $\beta_{2}$ para o modelo ZIB
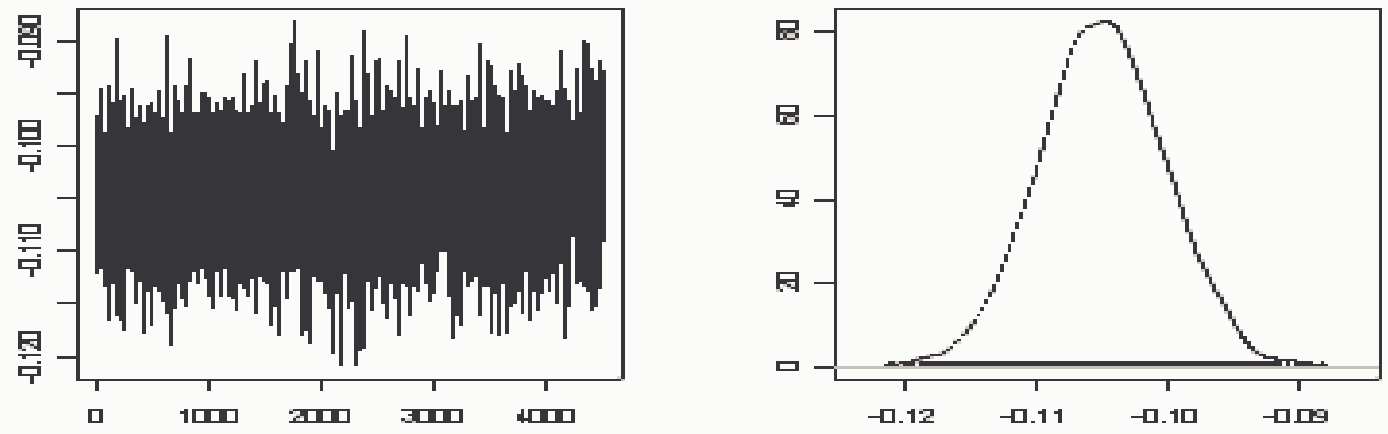

Figura 20 - Trajetória da cadeia ao longo das iterações e a aproximação da densidade a posteriori de $\beta_{3}$ para o modelo ZIB
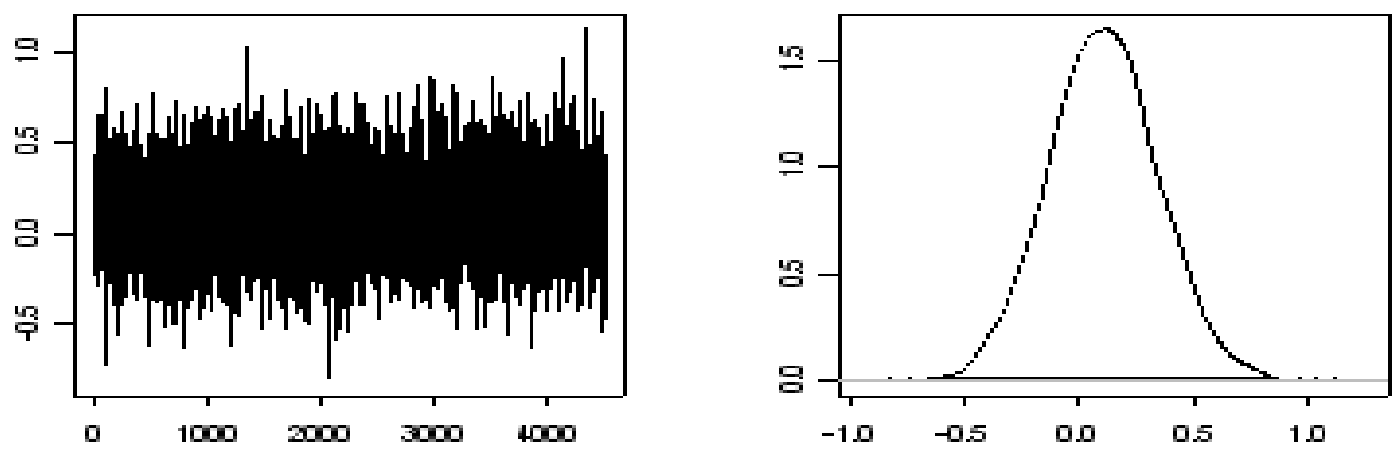

Figura 21 - Trajetória da cadeia ao longo das iterações e a aproximação da densidade a posteriori de $\gamma_{0}$ para o modelo ZIB 


\subsection{Comparação entre os modelos binomial, binomial trun- cado e ZIB}

Com base nos resultados do DIC e do fator de Bayes apresentados nas seções 4.1, 4.2 e 4.3 foram escolhidos, dentre os modelos ajustados, aqueles com preditores lineares polinomiais que melhor ajustaram-se aos dados e, então, obtidos os valores estimados para o número de ovos parasitados para os diferentes números de fêmeas conforme apresentado na Tabela 11 e na Figura 22.

Tabela 11. Número médio esperado de ovos parasitados usando diferentes números de fêmeas

\begin{tabular}{ccccc}
\hline $\log _{2}$ (número de fêmeas) & Observados & binomial & binomial truncado & ZIB \\
\hline 1 & 25,5 & 23,7 & 24,6 & 22,0 \\
2 & 6,6 & 8,2 & 7,9 & 12,0 \\
3 & 20,6 & 15,0 & 17,0 & 15,9 \\
4 & 23,9 & 32,3 & 30,2 & 28,9 \\
5 & 49,3 & 42,4 & 43,4 & 41,6 \\
6 & 33,8 & 36,5 & 36,7 & 44,1 \\
7 & 35,4 & 33,6 & 34,9 & 28,1 \\
\hline
\end{tabular}

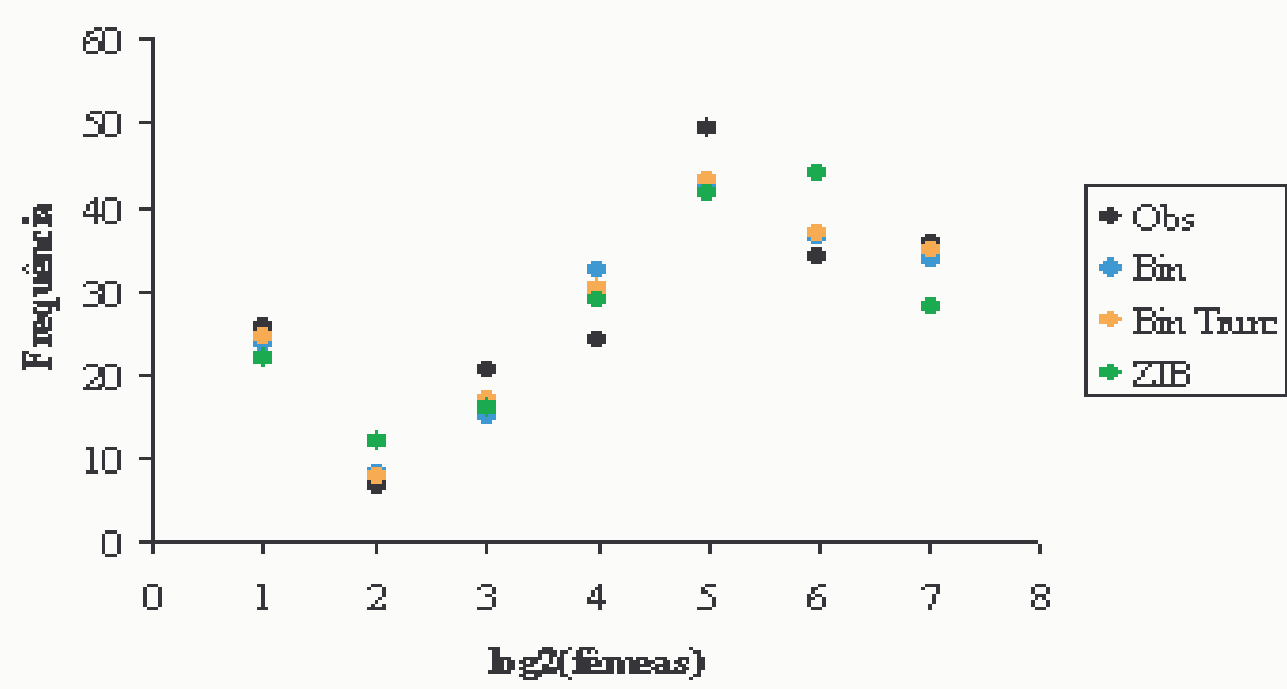

Figura 22 - Comparação entre os valores preditos pelas distribuições e o valor observado 
Nota-se que as distribuições binomial e binomial truncada apresentam valores preditos semelhantes e próximos dos valores observados considerando os diferentes número de fêmeas. Entretanto, para o logaritmo de 4 e 64 fêmeas o modelo ZIB superestima o número médio observado de ovos parasitados, e para o logaritmo de 128 fêmeas, esse modelo subestima o número médio observado de ovos parasitados. Vieira (1998) mostra, usando a inferência clássica, através do half normal plot com envelope simulado, que o modelo ZIB não se ajusta bem aos dados.

O número esperado de ovos parasitados, nesse conjunto de dados é de aproximadamente 27 ovos, portanto, se a distribuição binomial for usada é esperada uma probabilidade pequena de ovos não parasitados. Entretanto, foi observado que em 37 repetições $(52,9 \%$ ) nenhum ovo foi parasitado, tornando-se assim a distribuição binomial inadequada para esse conjunto de dados. A Figura 23 mostra a porcentagem observada de ovos não parasitados e estimados pelas distribuições binomial e ZIB.

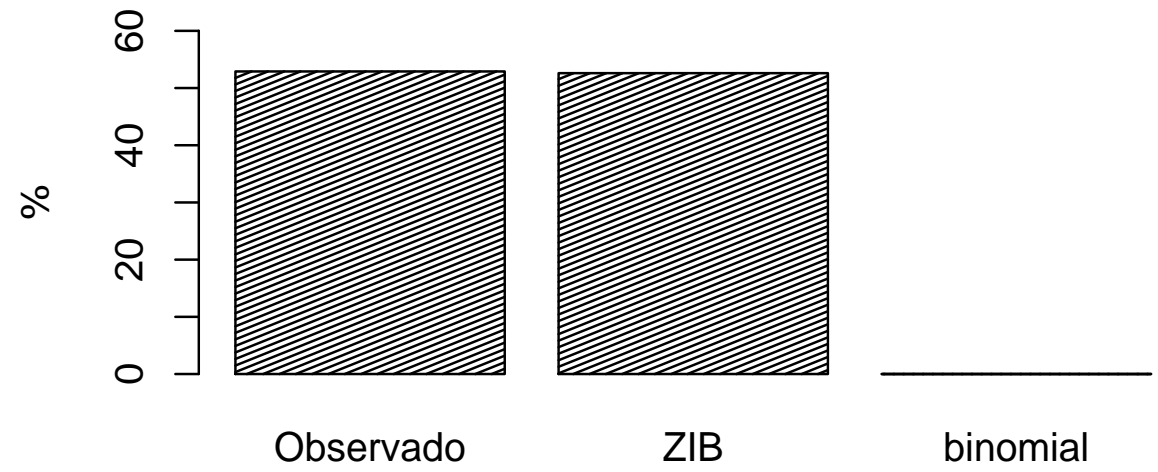

Figura 23 - Porcentagem observada e estimada de ovos não parasitados, segundo as distribuições binomial e ZIB

Considerando os resultados apresentados e discutidos nas seções 4.1, 4.2 e 4.3, deseja-se comparar as três distribuições usadas nesse trabalho. Na Tabela 12 é apresentada as estimativas de Monte Carlo das verossimilhanças marginais dos modelos binomial com preditor linear de quarto grau, binomial truncado com pre- 
ditor linear de terceiro grau e ZIB com preditores lineares de terceiro grau para $\boldsymbol{p}$ e constante para $\boldsymbol{w}$.

Tabela 12. Valores das verossimilhanças marginais para os modelos binomial, binomial truncado e ZIB

\begin{tabular}{cc}
\hline Modelos & $\hat{\pi}(y \mid M)$ \\
\hline binomial & $1,14.10^{-259}$ \\
binomial truncado & $1,117.10^{-62}$ \\
ZIB & $7,995.10^{-71}$ \\
\hline
\end{tabular}

Observa-se que a estimativa de Monte Carlo da verossimilhança marginal para o modelo binomial truncado apresentou-se bem superior aos modelos binomial e ZIB. Nota-se também, que o pior ajuste, segundo o fator de Bayes, é dado para o modelo binomial. Portanto, notou-se que o modelo padrão apresenta problemas no ajuste dos dados do ensaio de controle biológico.

Conforme os resultados apresentados na Tabela 12, utilizou-se o modelo binomial truncado com preditor linear de terceiro grau para determinar o número ideal de fêmeas que irá parasitar o maior número de ovos, sendo sua expressão dada por

$$
g\left(\hat{p}_{i}\right)=\log \left(\frac{\hat{p}_{i}}{1-\hat{p}_{i}}\right)=-0,1716-3,8459 x_{i}+1,2343 x_{i}^{2}-0,105 x_{i}^{3} .
$$

Maximizando-se $\hat{p}$ em relação a $x$, tem-se que

$$
\hat{p}=\frac{e^{0,97}}{1+e^{0,97}}
$$

em que o número máximo de ovos parasitados $\hat{\mu}=92,81$, com intervalo de confiança de $95 \%$ de probabilidade dado por $(82,94 ; 102,68)$, ocorre com 52 fêmeas.

Vieira (1998) verificou através do gráfico meio normal com envelopes simulados, que o modelo binomial truncado superdisperso com preditor linear de terceiro grau ajustou-se melhor aos dados, com intervalo de confiança dado por $(80,87 ; 103,48)$ para o número máximo de ovos parasitados $\hat{\mu}=93,13$, utilizando 52 
fêmeas. Portanto, nota-se que o DIC e o fator de Bayes parecem ser uma ferramenta bastante útil para a escolha do modelo probabilístico que deve ser utilizado para a modelagem de dados, porém a escolha do modelo também deve estar baseada no interesse do pesquisador sobre as observações nulas dos dados. Para esse conjunto de dados, as proporções de zeros não são informativas, já que o interesse está sobre o número máximo de ovos parasitados dado que pelo menos um ovo tenha sido parasitado. 


\section{CONCLUSÕES}

Nesse trabalho foram utilizados 3 modelos para a modelagem de dados de proporções com superdispersão e excesso de zeros. Para os modelos aqui usados, implementou-se o algoritmo Metropolis-Hastings no procedimento IML do programa SAS. Como critério de comparação da adequabilidade dos modelos foram utilizados o DIC e o fator de Bayes.

O modelo binomial inflacionado de zeros apesar de ter sido menos eficiente do que o modelo binomial truncado, quanto ao ajuste desse conjunto de dados, pode vir a ser útil para ajustar conjuntos de dados, em que o zero é de interesse do pesquisador.

A análise Bayesiana dos modelos usados para ajustar a variabilidade e o excesso de zeros, permite incorporar aos modelos informações prévias a respeito dos parâmetros, e isso muitas vezes produz inferências mais precisas.

O modelo binomial truncado superdisperso, com polinômio de terceiro grau, apresentou melhor ajuste aos dados e permitiu estimar o número máximo de ovos parasitados juntamente com o número ideal de fêmeas. Com isso, otimizou-se o trabalho do entomologista quanto ao tempo de execução do ensaio e a recursos financeiros.

Em relação a propostas futuras deste trabalho, pode-se considerar a análise Bayesiana de modelos com superdispersão mais complexas e estudar métodos de diagnósticos Bayesianos alternativos para a modelagem da superdispersão e do excesso de zeros. 
ANEXOS 
ANEXO A - Conjunto de dados referente ao experimento para medir a competição de fêmeas de T. galloi sob um número fixo (128) de ovos de $A$. kuehniella

\begin{tabular}{|c|c|c|}
\hline ovos parasitados & número total de ovos & número de fêmeas \\
\hline 0 & 128 & 2 \\
\hline 0 & 128 & 2 \\
\hline 0 & 128 & 2 \\
\hline 0 & 128 & 2 \\
\hline 0 & 128 & 2 \\
\hline 50 & 128 & 2 \\
\hline 65 & 128 & 2 \\
\hline 30 & 128 & 2 \\
\hline 62 & 128 & 2 \\
\hline 48 & 128 & 2 \\
\hline 0 & 128 & 4 \\
\hline 0 & 128 & 4 \\
\hline 0 & 128 & 4 \\
\hline 0 & 128 & 4 \\
\hline 0 & 128 & 4 \\
\hline 0 & 128 & 4 \\
\hline 0 & 128 & 4 \\
\hline 62 & 128 & 4 \\
\hline 3 & 128 & 4 \\
\hline 1 & 128 & 4 \\
\hline 0 & 128 & 8 \\
\hline 0 & 128 & 8 \\
\hline 0 & 128 & 8 \\
\hline 0 & 128 & 8 \\
\hline 0 & 128 & 8 \\
\hline 49 & 128 & 8 \\
\hline 19 & 128 & 8 \\
\hline 22 & 128 & 8 \\
\hline 51 & 128 & 8 \\
\hline 65 & 128 & 8 \\
\hline
\end{tabular}


ANEXO A - Conjunto de dados referente ao experimento para medir a competição de fêmeas de T. galloi sob um número fixo (128) de ovos de $A$. kuehniella

\begin{tabular}{|c|c|c|}
\hline ovos parasitados & número total de ovos & número de fêmeas \\
\hline 0 & 128 & 16 \\
\hline 0 & 128 & 16 \\
\hline 0 & 128 & 16 \\
\hline 0 & 128 & 16 \\
\hline 0 & 128 & 16 \\
\hline 57 & 128 & 16 \\
\hline 35 & 128 & 16 \\
\hline 52 & 128 & 16 \\
\hline 37 & 128 & 16 \\
\hline 58 & 128 & 16 \\
\hline 0 & 128 & 32 \\
\hline 0 & 128 & 32 \\
\hline 0 & 128 & 32 \\
\hline 0 & 128 & 32 \\
\hline 0 & 128 & 32 \\
\hline 120 & 128 & 32 \\
\hline 90 & 128 & 32 \\
\hline 86 & 128 & 32 \\
\hline 102 & 128 & 32 \\
\hline 95 & 128 & 32 \\
\hline 0 & 128 & 64 \\
\hline 0 & 128 & 64 \\
\hline 0 & 128 & 64 \\
\hline 0 & 128 & 64 \\
\hline 0 & 128 & 64 \\
\hline 0 & 128 & 64 \\
\hline 57 & 128 & 64 \\
\hline 77 & 128 & 64 \\
\hline 105 & 128 & 64 \\
\hline 99 & 128 & 64 \\
\hline
\end{tabular}


ANEXO A - Conjunto de dados referente ao experimento para medir a competição de fêmeas de T. galloi sob um número fixo (128) de ovos de $A$. kuehniella

\begin{tabular}{ccc}
\hline ovos parasitados & número total de ovos & número de fêmeas \\
\hline 0 & 128 & 128 \\
0 & 128 & 128 \\
0 & 128 & 128 \\
0 & 128 & 128 \\
38 & 128 & 128 \\
21 & 128 & 128 \\
82 & 128 & 128 \\
42 & 128 & 128 \\
90 & 128 & 128 \\
81 & 128 & 128 \\
\hline
\end{tabular}


ANEXO B - Programa WINBUGS para os resultados do DIC e do número efetivo de parâmetros, usando o modelo ZIB superdisperso com preditores lineres de terceiro grau para $\boldsymbol{p}$ e constante para $\boldsymbol{w}$

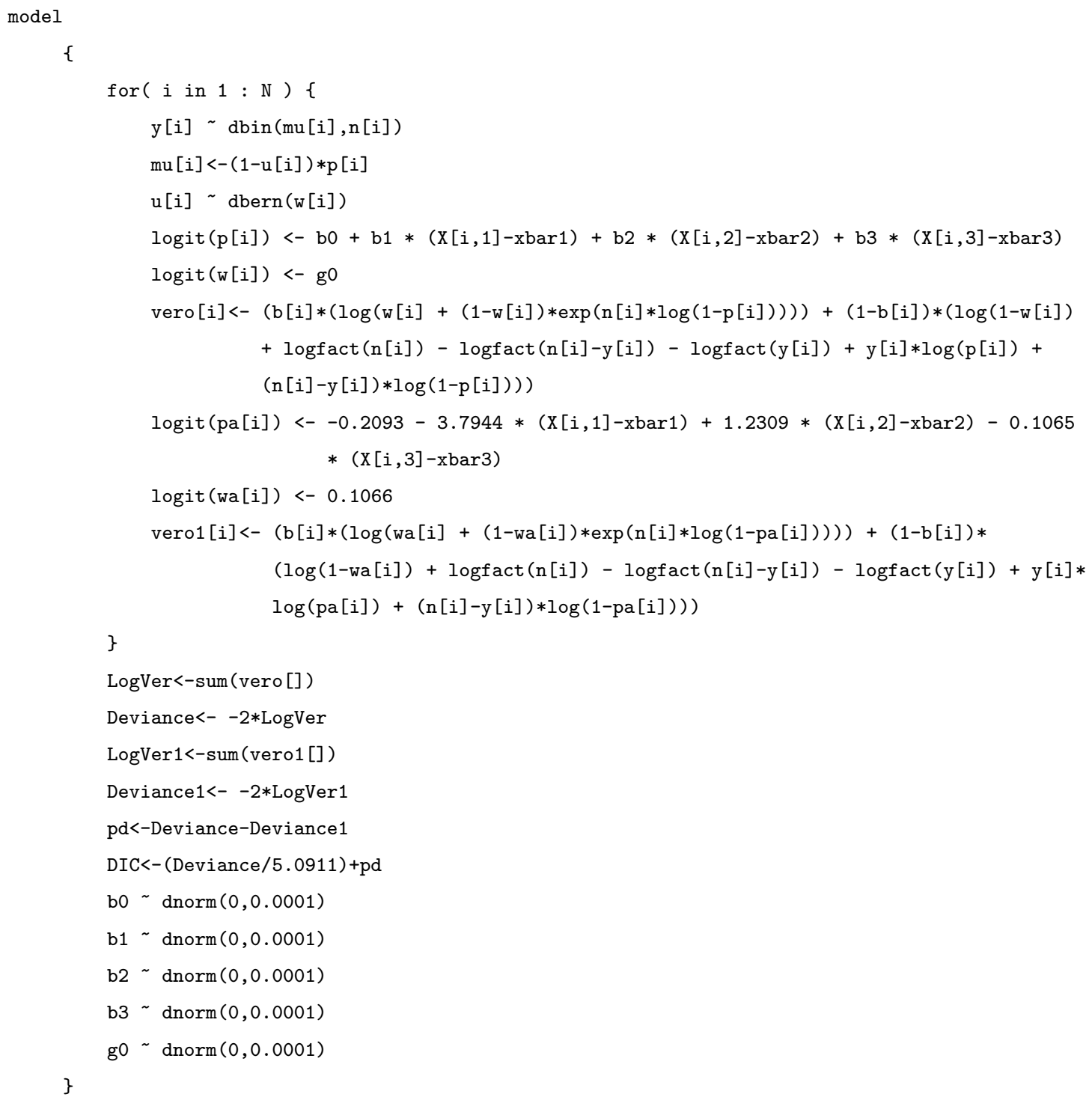


ANEXO C - Programa SAS para o algoritmo Metropolis-Hastings, usando o modelo ZIB superdisperso

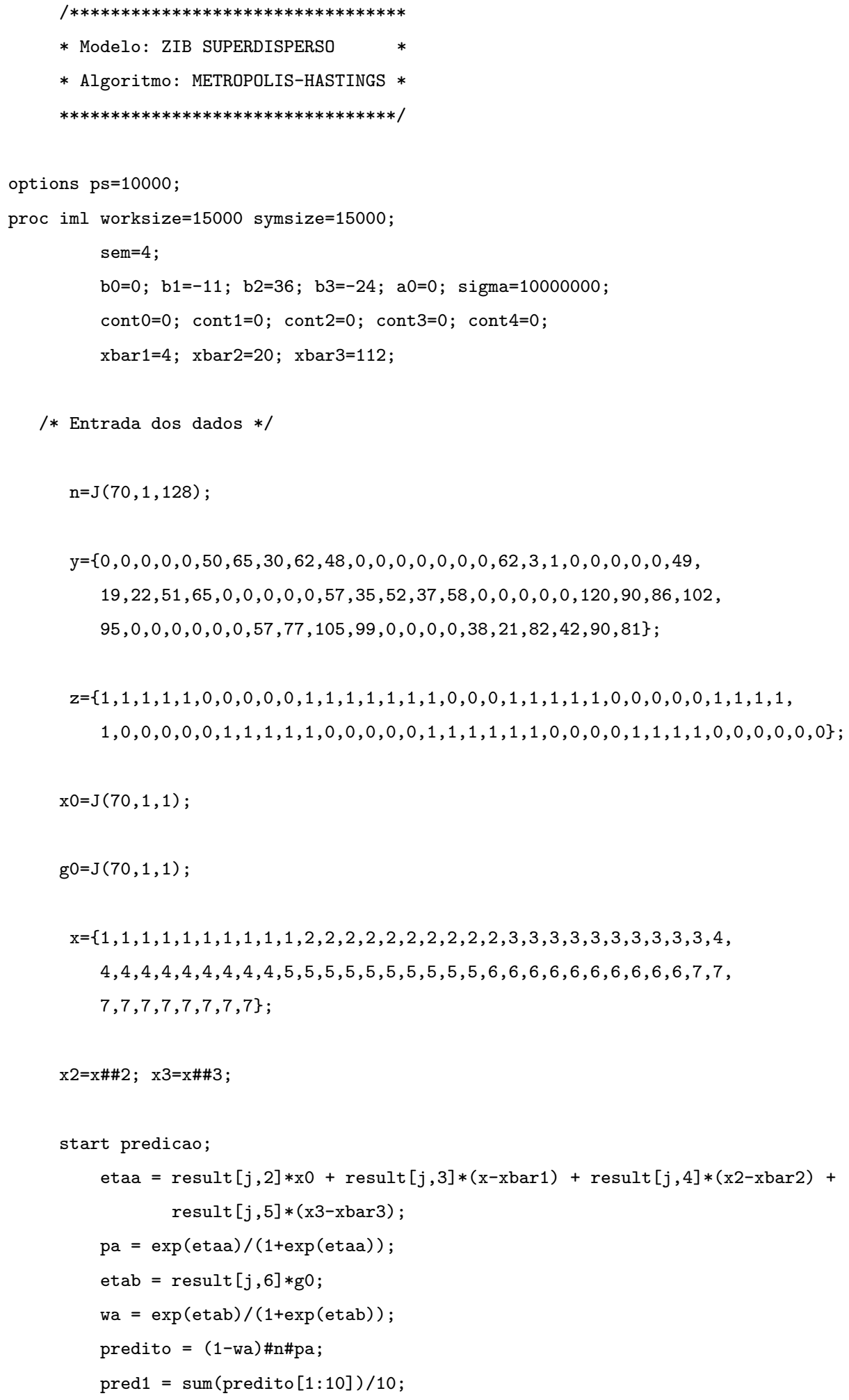


pred2 $=\operatorname{sum}($ predito $[11: 20]) / 10 ;$

pred3 $=\operatorname{sum}($ predito $[21: 30]) / 10 ;$

pred $4=\operatorname{sum}($ predito $[31: 40]) / 10 ;$

pred5 $=\operatorname{sum}($ predito $[41: 50]) / 10 ;$

pred $6=\operatorname{sum}($ predito $[51: 60]) / 10 ;$

pred7 $=\operatorname{sum}($ predito $[61: 70]) / 10 ;$

pred1 $f=$ pred $1 \mathrm{f} \mid$ | pred 1 ;

pred2f =pred2f | | pred2;

pred3f=pred3f | | pred3;

pred4f $=$ pred4f $\mid$ | pred4;

pred5 $f=$ pred5 $f \mid$ pred5;

pred6f $=$ pred $6 \mathrm{f}||$ pred 6 ;

pred7f $=$ pred7f $\mid$ |pred7;

finish;

start criterio;

k26=novo-velho;

$\mathrm{k} 27=\min (0, \mathrm{k} 26)$;

unif=unif orm (sem);

unif $=\log$ (unif);

finish;

start funcao;

b00 $=0+\operatorname{sqrt}(2) *$ normal (sem);

eta $00=b 00 * x 0+b 1 *((x-x b a r 1) / 3)+b 2 *((x 2-x b a r 2) / 29)+b 3 *((x 3-x b a r 3) / 231) ;$

eta $4=\mathrm{a} 0 * \mathrm{~g} 0$;

$\mathrm{p} 00=\exp ($ eta00) $/(1+\exp ($ eta 00$)) ;$

$\mathrm{w}=\exp ($ eta 4$) /(1+\exp ($ eta 4$))$;

prob00=z\# $(\mathrm{w}+(1-\mathrm{w}) \#(1-\mathrm{p} 00) \# \# \mathrm{n})+((1-\mathrm{z}) \#(1-\mathrm{w}) \# \mathrm{p} 00 \# \# \mathrm{y} \#(1-\mathrm{p} 00) \# \#(\mathrm{n}-\mathrm{y})) \# \#(5.5962 * *(-1))$;

pri00 $=-$ b00**2/(2*sigma) ;

novo $=\operatorname{sum}(\log ($ prob00 +0.0000001$))+$ pri00;

eta $0=b 0 * x 0+b 1 *((x-x b a r 1) / 3)+b 2 *((x 2-x b a r 2) / 29)+b 3 *((x 3-x b a r 3) / 231)$;

eta $4=\mathrm{a} 0 * \mathrm{~g} 0$;

$\mathrm{p} 0=\exp ($ eta 0$) /(1+\exp ($ eta 0$))$;

$\mathrm{w}=\exp ($ eta 4$) /(1+\exp ($ eta 4$))$;

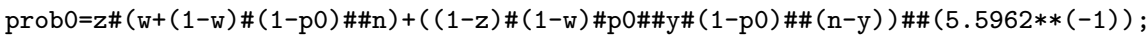

pri $0=-b 0 * * 2 /(2 *$ sigma $)$;

velho $=\operatorname{sum}(\log ($ prob0 +0.0000001$))+$ pri0 $;$

run criterio;

if unif $<=\mathrm{k} 27$ then $\mathrm{b} 0=\mathrm{b} 00$;

else $b 0=b 0$;

if unif $<=k 27$ then $\operatorname{cont} 0=(\operatorname{cont} 0+1)$.

b10 $=-11+\operatorname{sqrt}(2) *$ normal $($ sem $)$; 


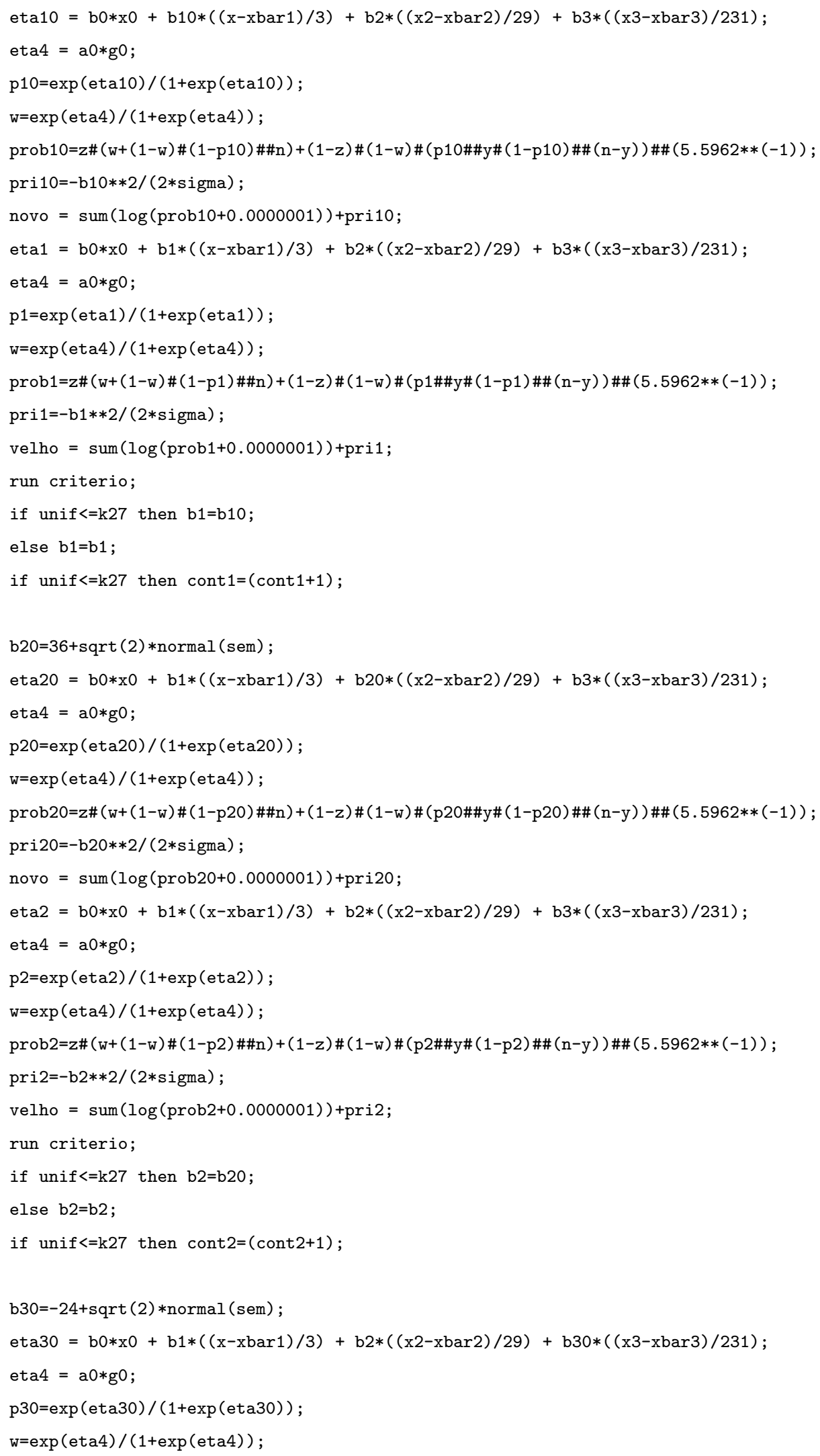




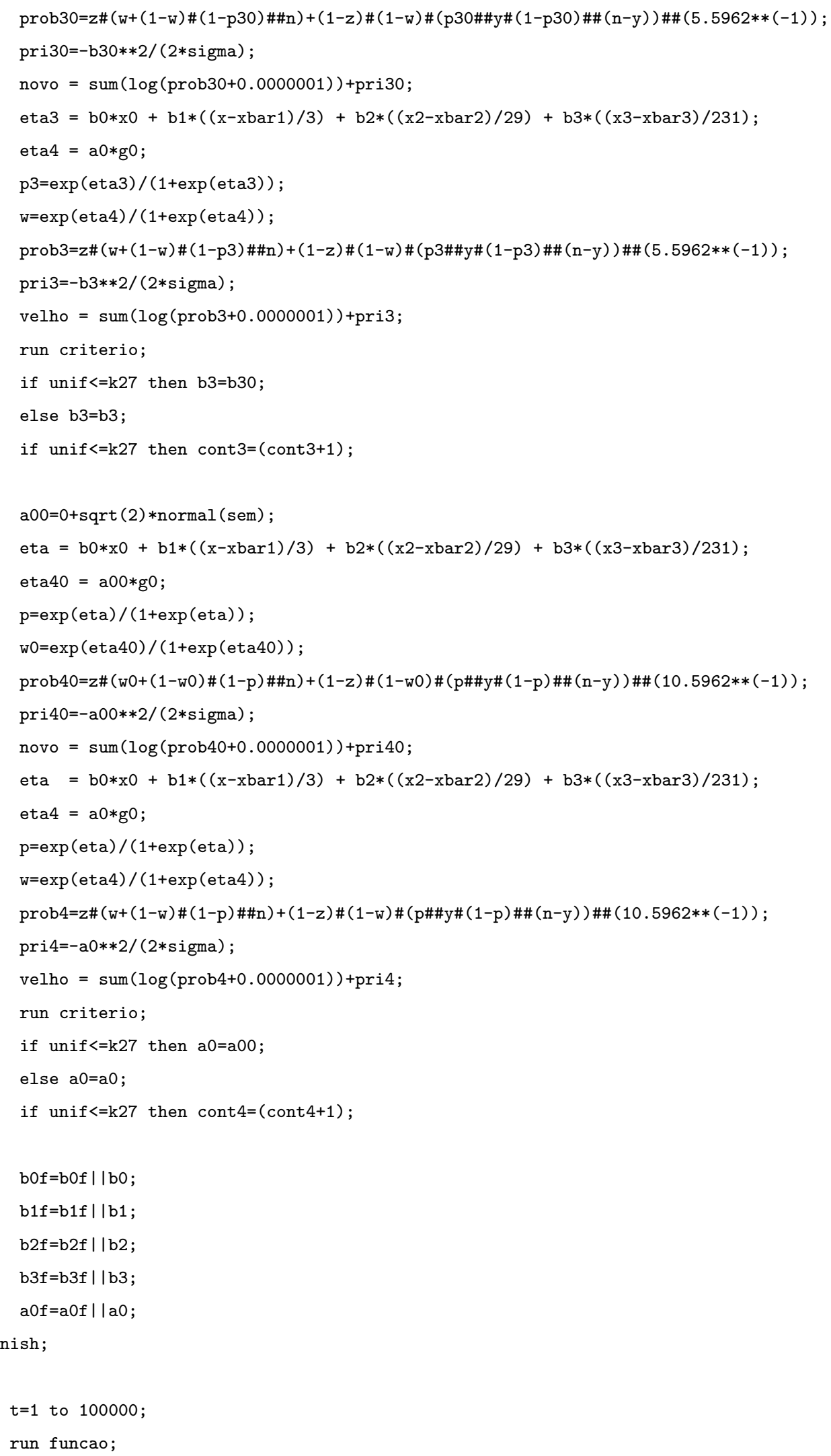


end

$\mathrm{s} 0=\mathrm{b} 0 \mathrm{f}^{\prime}$;

$\mathrm{s} 1=\mathrm{b} 1 \mathrm{f}^{\prime}$;

$\mathrm{s} 2=\mathrm{b} 2 \mathrm{f}^{\prime}$;

s3 $=$ b3f';

$\mathrm{s} 4=\mathrm{a} 0 \mathrm{f}^{\prime}$;

$\operatorname{arq} 1=\mathrm{s} 0|| \mathrm{s} 1|| \mathrm{s} 2|| \mathrm{s} 3|| \mathrm{s} 4$;

$r=\{b 0 \quad b 1 \quad b 2 \quad b 3 a 0\}$;

create arqui1 from arq1 [colname=r];

append from arq1;

result $t=$ shape $(0,4500,6)$;

in $=0$;

do $m=2000$ to 100000 by 21 ;

in $=i n+1$;

if in $>4500$ then goto fim;

result $[$ in, 1$]=$ in;

result $[$ in , 2] $=\mathrm{s} 0[\mathrm{~m}, 1]$;

result $[$ in , 3] $=\mathrm{s} 1[\mathrm{~m}, 1] / 3$;

result $[$ in , 4] $=\mathrm{s} 2[\mathrm{~m}, 1] / 29$;

result $[$ in , 5] $=\mathrm{s} 3[\mathrm{~m}, 1] / 231$;

result $[$ in, 6$]=s 4[m, 1]$;

fim:

end;

do $j=1$ to 4500 ;

run predicao;

end;

$\mathrm{z} 1=$ pred1f';

$\mathrm{z} 2=$ pred2f';

z3=pred3f ';

$z 4=$ pred4f';

$\mathrm{z} 5=$ pred5f';

$z 6=$ pred6f';

z7=pred7f ' ;

$\operatorname{arq} 26=z 1|| z 2|| z 3|| z 4|| z 5|| z 6|| z 7 ;$

$\mathrm{r} 2=\{$ predito 1 predito 2 predito 3 predito 4 predito 5 predito 6 predito $\}$;

create arqui3 from arq26 [colname=r2];

append from arq26;

arq16=result $[, 1]||$ result $[, 2]||$ result $[, 3]||$ result $[, 4]|| r e s u l t[, 5]|| r e s u l t[, 6]$;

$\mathrm{r} 1=\{$ in $\mathrm{b} 0 \mathrm{~b} 1 \mathrm{~b} 2 \mathrm{~b} 3 \mathrm{a} 0\}$;

create arqui2 from arq16 [colname=r1];

append from arq16; 
beta $0=\operatorname{sum}(\operatorname{result}[, 2]) /$ nrow $($ result $[, 2]) ;$

beta1=sum (result [,3])/nrow (result [,3] );

beta2 $=\operatorname{sum}(\operatorname{result}[, 4]) / \operatorname{nrow}(\operatorname{result}[, 4])$;

beta3 $=\operatorname{sum}(\operatorname{result}[, 5]) / \operatorname{nrow}(\operatorname{result}[, 5])$;

gama $0=\operatorname{sum}($ result $[, 6]) /$ nrow $($ result $[, 6])$;

print beta0 beta1 beta2 beta3 gama0;

print cont 0 cont 1 cont 2 cont 3 cont 4 in;

quit; 


\section{REFERÊNCIAS BIBLIOGRÁFICAS}

AKAIKE, H. Information theory and an extension of the maximum likelihood principle. In: INTERNATIONAL SYMPOSIUM OF INFORMATION THEORY, 2., Budapest, 1973. Proceedings. Budapest: Akademiai Kiàdio, 1973. p. $267-281$.

ATKINSON, A. Plots, transformations and regression: an introduction to graphical methods of diagnostic regression analysis. Oxford: Clarendon Press, 1985. 282p.

BAKKER, K.; BAGCHEE, S. N.; VAN ZWET, W.R.; MEELIS, E. Host discrimination in Pseudocoila Bochei (Hymenoptera: Cynipidae). Entomologia Experimentalis et Applicata, v.10, p.295 - 311, 1967.

BAKKER, K.; EIJSACKERS, H. J. P.; VAN LENTEREN, J. C.; MEELIS, E. Some models describing the distribuition of eggs of the parasite Pseudocoila Bocheii (Hymenoptera: Cynipidae) over its host, larvae of Drosophila melanogaster. Oecologia, v.10, p.29- 57, 1972.

BOTELHO, P.S.M. Tabela de vida ecológica e simulação da fase larval da Dia traea saccharalis (Fabr., 1974) (Lep.: Pyralidae). Piracicaba, 1985. 130p. Tese (Doutorado) - Escola Superior de Agricultura "Luiz de Queiroz", Universidade de São Paulo.

BRESLOW, N. Extra-poisson variation in log-linear models. Applied Statistics, v.33, p.38-44, 1984. 
CHIB, S.; GREENBERG, E. Understanding the Metropolis-Hastings. The American Statistician, v.49, p.327 - 335, 1995.

COLLETT, D. Modelling binary data. London: Chapman and Hall, 1991. 369p.

CORDEIRO, G.M. Modelos lineares generalizados. Campinas: UNICAMP, 1986. 286p.

COSTA, S.C. Modelos lineares generalizados mistos para dados longitudinais. Piracicaba, 2003. 110p. Tese (Doutorado) - Escola Superior de Agricultura "Luiz de Queiroz", Universidade de São Paulo.

COX, D.R.; MILLER, H.D. The theory of stochastic processes. London: Methuen, 1965. 408p.

DEMÉTRIO, C.G.B. Modelos lineares generalizados na experimentação agronômica. Piracicaba: ESALQ, Departamento de Ciências Exatas, 2001. $113 p$.

DEMÉTRIO, C.G.B.; HINDE, J. Half-Normal plots and overdispersion. GLIM Newsletter, v.27, p.19-26, 1997.

DEMPSTER, A.; LAIRD, N.M.; RUBIN, D.B. Maximum likelihood from incomplete data via the EM algorithm. Journal of the Royal Statistical Society, Series B, v.39, p.1 - 38, 1977.

DOBSON, A.J. An introduction to generalized linear models. London: Chapman and Hall, 2001. 225p.

FADDY, M.J. Extended Poisson process modelling and analysis of count data. Biometrical Journal, v.39, p.431 - 440, 1997.

FADDY, M.J. Statistics in ecology and environmental monitoring 2: decision making and risk assessment in biology. In: FLETCHER, D.J.; KAVALIERIS L.; 
MAINLY B.F.J. (Ed.) Stochastic models for analysis of species abundance data. Dunedin: University of Otago Press, 1998. cap.2, p.33 - 40.

FINNEY, D.J. Probit analysis. Cambridge: Cambridge University Press, 1947. $333 \mathrm{p}$.

FRIENDLY, M. Half normal plot for generalized linear models www.math. yorku.ca/SCS/vcd/halfnorm.html. (09 nov. 2000).

GANIO, L.M.; SCHAFER, D.W. Diagnostics for overdispersion. Journal of the American Statistical Association, v.87, p.795-804, 1992.

GELFAND, A.E.; SMITH, A.F.M. Sampling based approaches to calculating marginal densities. Journal of the American Statistical Association, v.85, p.398 - 409, 1990 .

GELMAN, A.; CARLIN, J.B.; STERN, H.S.; RUBIN, D.B. Bayesian data analysis. 2.ed. London: Chapman-Hall; CRC Press, 2003. 668p.

GHOSH, S.K..; MUKHOPADHYAY, P.; LU, J.C. Bayesian analysis of zeroinflated regression models. www.stat.ncsu.edu $\backslash \sim \operatorname{sgosh2.~(10~jan.~2002).~}$

GRIZZLE, J.E., STARMER, C.F., KOCH, G.G. Analysis of categorical data by linear models. Biometrics, v.25, p.489 - 504, 1969.

GROGGER, J.T.; CARSON, R.T. Models for truncated counts. Journal of Applied Econometrics, v.6, p.225-238, 1991.

GUPTA, P.R.; TRIPATHI, R. Analysis of zero-adjusted count data. Computational Statistics and Data Analysis, v.23, p.207-218, 1996.

GURMU, S. Generalized hurdle count data regression models. Econometrics Letters, v.58, p.263-268, 1998.

GURMU, S.; TRIVEDI, P.K. Excess zeros in count models for recreational trips. Journal of Business and Economic Statistics, v.14, p.469-477, 1996. 
HALL, D.B. Zero-inflated Poisson and binomial regression with random effects: a case study. Biometrics, v.56, p.1030 - 1039, 2000.

HEIDELBERGER, P.; WELCH P.D. Simulation run length control in the presence of an initial transient. Operations Research, v.31, p.1109 - 1144, 1983.

HEILBRON, D.C. Zero-altered and other regression models for count data with added zeros. Biometrical Journal, v.36, p.531 - 547, 1994.

HINDE, J.; DEMÉTRIO, C.G.B. Overdispersion: models and estimation. Computational Statistics and Data Analysis, v.27, p.151 - 170, 1998a.

HINDE, J.; DEMÉTRIO, C.G.B. Overdispersion: models and estimation. São Paulo: ABE, 1998b. 73p.

HINDE, J.; DEMÉTRIO, C.G.B. Overdispersion: models and estimation. Berkeley: University of California, 2000. 110p.

JANSAKUL, N. Some aspects of modelling overdispersed and zero-inflated count data. Exeter, 2001. 306p. Thesis (Ph.D.) - University of Exeter.

KASS, R.E.; RAFTERY, A.E. Bayes factors and model uncertainty. Journal of the American Statistical Association, v.90, p.773 - 775, 1995.

KASS, R.E.; TIERNEY, L.; KADANE, J.B. Aproximate methods for assessing influence and sensitivity in Bayesian analysis. Biometrika, v.76, p.663 - 674, 1989.

LAMBERT, D. Zero-inflated Poisson regression, with na application to defects in manufacturing. Technometrics, v.34, p.1 - 14, 1992.

LAWLESS, J.F. Negative binomial and mixed Poisson regression. The Canadian Journal of Statistics, v.15, p.209-225, 1987.

LIANG, K.Y.; McCULLAGH, P. Case studies in binary dispersion. Biometrics, v.49, p.623-630, 1993. 
LINDSEY, J.K. Modelling frequency and count data. Oxford: Oxford University Press, 1995. 304p.

LINDSEY, J.K. Applying generalized linear models. New York: SpringerVerlag, 1997. 256p.

McCULLAGH, P.; NELDER, J.A. Generalized linear models. 2.ed. London: Chapman-Hall, 1989. 511p.

MEILIJSON, I. A fast improvement to the EM algorithm on its own terms. Journal of the Royal Statistical Society, Series B, v.51, n.1, p.127-138, 1989.

MORGAN, R.W. Some stochastic models to describe the fertilization of an egg. Applied Statistics, v.24, p.137 - 138, 1975.

MULLAHY, J. Specification and testing of some modified count data models. Journal of Econometrics, v.33, p.341 - 365, 1986.

MULLAHY, J. Heterogeneity, excess zeros, and the structure of count data models. Journal of Applied Econometrics, v.12, p.337 - 350, 1997.

NELDER, J.A.; WEDDERBURN, R.W.M. Generalized linear models. Journal of the Royal Statistical Society. Series A, Oxford, v.135, p.370 - 384, 1972.

PARRA, J.R.P. Técnicas de criação de Anagasta kuehniella hospedeiro alternativo para a produção de Trichogramma. In: PARRA, J.R.P., ZUCCHI, R.A. (Ed.) Trichogramma e o controle biológico aplicado. Piracicaba: FEALQ, 1997. cap. 4, p.121-150.

PAULA, G.A. Modelos de regressão com apoio computacional. São Paulo: USP, IME, 2002. 235p.

RAFTERY, A.E.; LEWIS, S.M. One long run with diagnostics: implementation strategies for Markov chain Monte Carlo. Statistical Science, v.7, p.493 - 497, 1992. 
RIDOUT, M.S.; DEMÉTRIO, C.G.B. Generalized linear models for positive count data. Revista de Matemática e Estatística, v.10, p.139 - 148, 1992.

RIDOUT, M.S.; DEMÉTRIO, C.G.B; HINDE, J.P. Models for count data with many zeros. In: INTERNATIONAL BIOMETRIC CONFERENCE, Cape Town, 1998. Proceedings, Cape Town: IBC, 1998. p.1 - 13.

ROGERS, D. A model for avoidance of superparasitism by solitary insect parasitoids. Journal of Animal Ecology, v.44, p.623 - 628, 1975.

SCHWARZ, G. Estimating the dimension of a model. Annals of Statistics, v.6, p.461 - 464, 1978.

SMITH, H.S. An attempt to redefine the host relationships exhibited by entomophagous insects. Journal of Economic Entomology v.9, p.447 - 486, 1916.

SPIEGELHALTER, D.J.; BEST, N.G.; CARLIN, B.P. Bayesian deviance, the effective number of parameters, and the comparasion of arbitrarily complex models: technical report. Cambridge: MRC Biostatistics, 1998. 31p.

VIEIRA, A.M.C. Modelos para dados de proporções com superdispersão aplicados ao controle biológico. Piracicaba, 1998. 61p. Dissertação (Mestrado) - Escola Superior de Agricultura "Luiz de Queiroz", Universidade de São Paulo.

VIEIRA, A.M.C.; HINDE, J. ; DEMÉTRIO, C.G.B. Zero-inflated proportion data models applied to a biological control assay. Journal of Applied Statistics, v.27, p.373-389, 2000 .

WILLIAMS, D.A. Extra-binomial variation in logistic linear models. Journal of Applied Statistics, v.31, p.144-148, 1982.

WILLIAMS, D.A. Generalized linear model diagnostics using the deviance and "single-case deletions". Journal of Applied Statistics, v.36, p.181-191, 1987. 
YAN, K.K.W.; LEE, HH. Zero-inflated Poisson regression with random effects to evaluate an occupational injury prevention programme. Statistics in Medicine, v.20, p.2907-2920, 2001. 